\title{
BOUNDED REALIZATION OF $l$-GROUPS OVER GLOBAL FIELDS
}

\author{
The method of Scholz and Reichardt
}

\author{
WULF-DIETER GEYER AND MOSHE JARDEN ${ }^{1}$
}

\begin{abstract}
We use the method of Scholz and Reichardt and a transfer principle from finite fields to pseudo finite fields in order to prove the following result. THEOREM Let $G$ be a group of order $l^{n}$, where $l$ is a prime number. Let $K_{0}$ be either a finite field with $\left|K_{0}\right|>l^{4 n+4}$ or a pseudo finite field. Suppose that $l \neq \operatorname{char}\left(K_{0}\right)$ and that $K_{0}$ does not contain the root of unity $\zeta_{l}$ of order $l$. Let $K=K_{0}(t)$, with $t$ transcendental over $K_{0}$. Then $K$ has a Galois extension $L$ with the following properties: (a) $\mathcal{G}(L / K) \cong G$; (b) $L / K_{0}$ is a regular extension; (c) genus $(L)<\frac{1}{2} n l^{2 n}$; (d) $K_{0}[t]$ has exactly $n$ prime ideals which ramify in $L$; the degree of each of them is $\left[K_{0}\left(\zeta_{l^{n}}\right): K_{0}\right] ;(\mathrm{e})(t)_{\infty}$ totally decomposes in $L ;(\mathrm{f})$ $L=K(x)$, with $\operatorname{irr}(x, K)=X^{l^{n}}+a_{1}(t) X^{l^{n}-1}+\cdots+a_{l^{n}}(t), 0<\operatorname{deg}\left(a_{1}(t)\right) \leq$ $\frac{1}{2} n l^{2 n}$ and $\operatorname{deg}\left(a_{i}(t)\right)<\operatorname{deg}\left(a_{1}(t)\right)$ for $i=1, \ldots, l^{n}$.
\end{abstract}

\section{Introduction}

Scholz [Sch] proved that if $l$ is an odd prime, then each $l$-group occurs as a Galois group over $\mathbb{Q}$. Here $G$ is an l-group if the order of $G$ is a power of $l$. Independently, Reichardt [Rei] gave a simpler and shorter proof to the same theorem. One can find a modern presentation of Reichardt's proof in Serre's course on Galois theory [Se1, §2.1].

The reason why the method of Scholz and Reichardt does not work for $l=2$ is that the primitive root of unity of order 2 , namely -1 , belongs to $\mathbb{Q}$. The same reason forced Rzedowski-Calderón and Villa-Salvador [RCV] to exclude all primes $l$ with $\zeta_{l} \in \mathbb{F}_{q}$, when they proved that each $l$-group occurs as a Galois group over $\mathbb{F}_{q}(t)$. Here $q$ is a power of a prime $p \neq l$ and $\zeta_{l}$ is a primitive root of unity of order $l$.

Shafarevich [Sh1] has overcome this difficulty. He used refined combinatorial arguments to prove that for an arbitrary prime number $l$, for each number field $K$, and for each $l$-group $G$, there exists a Galois extension $L$

\footnotetext{
Received July 10, 1995.

${ }^{1}$ This research was supported by The Israel Science Foundation administered by The Israel Academy of Sciences and Humanities.
} 
of $K$ such that $\mathcal{G}(L / K) \cong G$. In a later work [Sh2], Shafarevich pointed out how to correct an incomplete group theoretic argument in his earlier work for the case $l=2$.

However, Shafarevich had to pay a price for his generalization. The combinatorial arguments forced him to allow an exponentially growing number of primes of $K$ which may ramify in $L$. In contrast, as Serre [Se1, p. 9] emphasizes, the method of Scholz and Reichardt gives for a group $G$ of order $l^{n}$, with $l$ odd, a Galois extension $L$ of $\mathbb{Q}$ in which only $n$ primes ramify.

Although Rzedowski-Calderón and Villa-Salvador [RCV] use the method of Scholz and Reichardt they do not try to bound the number of ramified primes. Indeed for a given $l$-group $G$ with $l \nmid q$ and $\zeta_{l} \notin \mathbb{F}_{q}$, they construct a Galois extension $L$ of $\mathbb{F}_{q}(t)$ such that $\mathcal{G}\left(L / \mathbb{F}_{q}(t)\right) \cong G$ and the genus of $L$ is large. Here and in the sequel, $t$ is a transcendental element over the base field. By the Hurwitz-Riemann genus formula, this means that the number of primes of $\mathbb{F}_{q}(t)$ which ramify in $L$ is also large.

The goal of this work is to use the method of Scholz and Reichardt to realize each $l$-group $G$ over an arbitrary global field with bounded ramification.

THEOREM A. Let $K$ be a global field and let $l$ be a prime number such that $l \neq \operatorname{char}(K)$ and $\zeta_{l} \notin K$. Then there exists a nonnegative integer $r=r(K)$ such that for each group $G$ of order $l^{n}$ there exists a Galois extension $L$ of $K$ with $\mathcal{G}(L / K) \cong G$ and $|\operatorname{Ram}(L / K)| \leq n+r$.

Here $\operatorname{Ram}(L / K)$ denotes the set of primes of $K$ which ramify in $L$. If $K=\mathbb{Q}$ or $K=\mathbb{F}_{q}(t)$, then $r(K)=0$. In the former case we therefore reproduce the result of [Se1]. In the latter case we improve the result of [RCV].

The extension $L / K$ which Theorem A gives can actually be constructed to satisfy given 'local conditions'. See Theorem 7.4 for the exact formulation.

Serre uses cyclotomic fields in his proof. Rzedowski-Calderón and VillaSalvador use Carlitz' analog of cyclotomic fields over $\mathbb{F}_{q}(t)$. Both type of fields are useless for the construction of $l$-extensions with bounded ramification over an arbitrary global field $K$. We replace their use by a systematic application of class field theory. 
In the function field case, we supplement Theorem A with bounds on various invariants of the extension $L / K$. We prove that for each large multiple $k$ of $\left[\mathbb{F}_{q}\left(\zeta_{l^{n}}\right): \mathbb{F}_{q}\right]$, the field $L$ can be chosen such that $\operatorname{deg}(\mathfrak{p})=k$ for each prime $\mathfrak{p} \in \operatorname{Ram}(L / K)$ and

$$
2 g_{L}-2 \leq l^{n}\left(2 g_{K}-2+(n+r(K)) k\right) .
$$

Here $g_{K}$ (resp., $g_{L}$ ) is the genus of $K$ (resp., $L$ ). See Theorem 8.6 for more details.

The bounds of Theorem 9.6 become more explicit if $K=\mathbb{F}_{q}(t)$. For example, Theorem 9.1 improves (0.1) in this case to

$$
2 g_{L}-2 \leq l^{n}(n k-2) .
$$

Theorem 10.5 does even better under the assumption that $q>l^{4 n+4}$. It produces absolutely irreducible polynomials $f, g \in \mathbb{F}_{q}(T, X)$ with coefficients of bounded degrees such that $L$ is the splitting field over $K=\mathbb{F}_{q}(t)$ of both $f(t, X)$ and $g(t, X)$ and $\operatorname{Ram}(L / K)$ consists of those prime of $K$ which divide the discriminants of both $f(t, X)$ and $g(t, X)$. Of course, $\mathcal{G}(L / K) \cong G$. Moreover, $(t)_{\infty}$ totally decomposes in $L, g_{L} \leq \frac{1}{2} n l^{2 n}$, $|\operatorname{Ram}(L / K)|=n$, and $\operatorname{deg}(\mathfrak{p})=\left[\mathbb{F}_{q}\left(\zeta_{l^{n}}\right): \mathbb{F}_{q}\right]$ for each $\mathfrak{p} \in \operatorname{Ram}(L / K)$.

It is therefore possible to write down a sentence $\theta(l, n)$ in the first order language of the theory of fields, such that if $q>l^{4 n+4}$, then $K=\mathbb{F}_{q}(t)$ has a Galois extension as in the preceding paragraph.

This has an immediate consequence for the infinite models of the theory of finite fields. They are called pseudo finite fields. For example, each nonprincipal ultraproduct of finite fields is pseudo finite. Also, if $F$ is a countable Hilbertian field (e.g., $F=\mathbb{Q}$ or $F=\mathbb{F}_{q}(t)$ ), then $\tilde{F}(\sigma)$ is pseudo finite for almost all $\sigma$ in the absolute Galois group $G(F)$ of $F$ [Ja1, Thm. 3.5]. Here $\tilde{F}(\sigma)$ is the fixed field of $\sigma$ in the algebraic closure $\tilde{F}$ of $F$.

TheOREM B. Let $K_{0}$ be a pseudo finite field and let $l$ be a prime number such that $l \nmid \operatorname{char}\left(K_{0}\right)$ and $\zeta_{l} \notin K_{0}$. Let $G$ be. a group of order $l^{n}$. Then $K=K_{0}(t)$ has a Galois extension $L$ which is regular over $K_{0}$ such that $\mathcal{G}(L / K) \cong G,|\operatorname{Ram}(L / K)|=n, g_{L} \leq \frac{1}{2} n l^{2 n},(t)_{\infty}$ totally decomposes in $L$, and $\operatorname{deg}(\mathfrak{p})=\left[K_{0}\left(\zeta_{l^{n}}\right): K_{0}\right]$ for each $\mathfrak{p} \in \operatorname{Ram}(L / K)$.

Note that each pseudo finite field $K_{0}$ is PAC. That is, each absolutely irreducible variety over $K_{0}$ has a $K_{0}$-rational point. It is known for an 
arbitrary PAC field $K_{0}$ and for each finite group $G$ that there exists a Galois extension $L$ of $K_{0}(t)$ which is regular over $K_{0}$ such that $\mathcal{G}(L / K) \cong G$. This was first proved by Fried and Völklein [FrV] in characteristic 0 . In the general case it follows from a theorem of Harbater [Ja2, Thm. 2.6] which has been recently reproved in an elementary way by Haran and Völklein $[\mathrm{HaV}]$. However, none of the proofs of this theorem supplies a bound for $|\operatorname{Ram}(L / K)|$. Theorem $\mathrm{B}$ does it in a very special case.

\section{$\S 1$. Global fields}

Consider a global field $K$. Thus, $K$ is either a number field, that is, a finite extension of $\mathbb{Q}$, or $K$ is a function field, that is, $K$ is a regular extension of transcendence degree 1 of a finite field $\mathbb{F}_{q}$ with $q$ elements. We denote the set of primes of $K$ by $\mathbb{P}=\mathbb{P}(K)$. In the number field case, $\mathbb{P}$ has a finite subset $\mathbb{P}_{\infty}$, the set of archimedean primes, which correspond to the embeddings of $K$ into $\mathbb{C}$. Each archimedean prime $\mathfrak{p}$ is a divisor of the unique archimedean prime $\infty$ of $\mathbb{Q}$ and we write $\mathfrak{p} \mid \infty$. All other primes of $K$ are nonarchimedean. In particular, if $K$ is a function field, then $K$ has only nonarchimedean primes and we let $\mathbb{P}_{\infty}=\emptyset$.

Fix a prime number $l$ which does not divide $\operatorname{char}(K)$. For each $n$ choose a root of unity $\zeta_{l^{n}}$ of order $l^{n}$. Most of our results will assume that $\zeta_{l} \notin K$. If $K$ is a number field, we denote the finite subset of $\mathbb{P}$ that consists of all prime divisors of $l$ by $\mathbb{P}_{l}$. If $K$ is a function field, we let $\mathbb{P}_{l}=\emptyset$.

Denote the completion of $K$ at a prime $\mathfrak{p}$ by $K_{\mathfrak{p}}$ and let $\bar{K}_{\mathfrak{p}}$ be its residue field. If $\mathfrak{p}$ is archimedean, then $K_{\mathfrak{p}}$ is either $\mathbb{R}(\mathfrak{p}$ is real) or $\mathbb{C}(\mathfrak{p}$ is complex). In each case we let $U_{\mathfrak{p}}=K_{\mathfrak{p}}^{\times}$be the multiplicative group of $K_{\mathfrak{p}}$ and set $\pi_{\mathfrak{p}}=1$. If $\mathfrak{p}$ is nonarchimedean, then $K_{\mathfrak{p}}$ is a complete discrete valuation field. We denote its normalized valuation by $v_{\mathfrak{p}}$ and choose a prime element $\pi_{\mathfrak{p}}$ in $K_{\mathfrak{p}}$, that is $v_{\mathfrak{p}}\left(\pi_{\mathfrak{p}}\right)=1$. Let $U_{\mathfrak{p}}$ be the group of units of $K_{\mathfrak{p}}$ and $U_{\mathfrak{p}, 1}$ its group of 1 -units. Then $K_{\mathfrak{p}}^{\times} \cong\left\langle\pi_{\mathfrak{p}}\right\rangle \times U_{\mathfrak{p}}$ and $U_{\mathfrak{p}} / U_{\mathfrak{p}, 1}$ is isomorphic to $\bar{K}_{\mathfrak{p}}^{\times}$. If $K$ is a number field, then $\bar{K}_{\mathfrak{p}}$ is a finite extension of $\mathbb{F}_{p}$, where $p$ is the rational prime that lies under $\mathfrak{p}$. If $K$ is a function field, then $\bar{K}_{\mathfrak{p}}$ is a finite extension of $\mathbb{F}_{q}$ and $\left[\bar{K}_{\mathfrak{p}}: \mathbb{F}_{q}\right]=\operatorname{deg}(\mathfrak{p})$. In both cases $U_{\mathfrak{p}, 1}$ is a pro- $p$ group, where $p=\operatorname{char}\left(\bar{K}_{\mathfrak{p}}\right)$.

The group $I_{K}$ of ideles of $K$ is the restricted product of the multiplica- 
tive groups $K_{\mathfrak{p}}^{\times}$with respect to the subgroups $U_{\mathfrak{p}}$. Thus

$$
I_{K}=\left\{\alpha \in \prod_{\mathfrak{p} \in \mathbb{P}} K_{\mathfrak{p}}^{\times} \mid \alpha_{\mathfrak{p}} \in U_{\mathfrak{p}} \text { for all but finitely many } \mathfrak{p} \in \mathbb{P}\right\} .
$$

A basis for the topology of $I_{K}$ consists of all sets $\prod V_{\mathfrak{p}}$ such that $V_{\mathfrak{p}}$ is open in $K_{\mathfrak{p}}^{\times}$and $V_{\mathfrak{p}}=U_{\mathfrak{p}}$ for almost all $\mathfrak{p}$. In particular, the group of unit ideles is open in $I_{K}$ :

$$
U=\prod_{\mathfrak{p} \in \mathbb{P}} U_{\mathfrak{p}}
$$

This group is the kernel of the divisor map div: $I_{K} \rightarrow \operatorname{Div}(K)=\bigoplus_{\mathfrak{p} \nmid \infty} \mathbb{Z} \cdot \mathfrak{p}$ which is defined by $\operatorname{div}(\alpha)=\sum_{\mathfrak{p} \nmid \infty} v_{\mathfrak{p}}\left(\alpha_{\mathfrak{p}}\right) \mathfrak{p}$. The image of the divisor map is the group of divisors $\operatorname{Div}(K)$ of $K$.

One embeds $K^{\times}$diagonally in $I_{K}$ and calls $C_{K}=I_{K} / K^{\times}$the idele class group of $K$. Its factor by $U K^{\times} / K^{\times}$is the ideal class group of $K$ :

$$
\mathrm{Cl}(K)=I_{K} / U K^{\times}=\operatorname{Div}(K) / \operatorname{div}\left(K^{\times}\right) .
$$

If $K$ is a number field, then $\mathrm{Cl}(K)$ is a finite abelian group, whose order $h_{K}$ is the class number of $K$. If $K$ is a function field, then we define the degree function for ideles $\operatorname{deg}: I_{K} \rightarrow \mathbb{Z}$ by $\operatorname{deg}(\alpha)=\sum_{\mathfrak{p} \in \mathbb{P}} v_{\mathfrak{p}}\left(\alpha_{\mathfrak{p}}\right) \operatorname{deg}(\mathfrak{p})$ and consider the group of ideles of degree 0 :

$$
I_{K}^{0}=\left\{\alpha \in I_{K} \mid \operatorname{deg}(\alpha)=0\right\}
$$

The product formula implies that $K^{\times} \leq I_{K}^{0}$. Also, $U \leq I_{K}^{0}$. So, we may consider the group of idele classes of degree $0: \mathrm{Cl}_{0}(K)=I_{K}^{0} / U K^{\times}$. Then $\mathrm{Cl}(K) / \mathrm{Cl}_{0}(K) \cong \mathbb{Z}$, but $\mathrm{Cl}_{0}(K)$ is a finite abelian group whose order $h_{K}$ is the class number of $K$. More important for us is the $l$-class rank of $K$ :

$$
\operatorname{rank}_{l}(K)=\operatorname{dim}_{\mathbb{F}_{l}} \mathrm{Cl}(K)_{l}
$$

Here $\mathrm{Cl}(K)_{l}$ is the $l$-torsion part of the finite abelian group $\mathrm{Cl}(K)$.

To each finite nonempty subset $S$ of $\mathbb{P}$ that contains $\mathbb{P}_{\infty}$ one associates the group $K_{S}$ of $S$-units. Thus $K_{S}$ consists of all elements $x \in K$ such that $v_{\mathfrak{p}}(x)=0$ for all $\mathfrak{p} \notin S \cup \mathbb{P}_{\infty}$. By Dirichlet's unit theorem, $K_{S} \cong \mu_{K} \times \mathbb{Z}^{|S|-1}$ where $\mu_{K}$ is the finite cyclic group of roots of unity of $K$ [CaF, p. 72]. If $K$ is a function field, then $\mu_{K}=\mathbb{F}_{q}^{\times}$. If $K$ is a number field and $S=\mathbb{P}_{\infty}$, we 
call $\operatorname{rank}_{\infty}(K)=\left|\mathbb{P}_{\infty}\right|-1$ the unit rank of $K$ and $E_{K}=K_{\mathbb{P}_{\infty}}$ is the group of units of $K$. If $K$ is a function field, we let $E_{K}=\mu_{K}$ and $\operatorname{rank}_{\infty}(K)=0$. The group of $S$-ideles of $K$ is the direct product

$$
I_{K, S}=\prod_{\mathfrak{p} \in S} K_{\mathfrak{p}}^{\times} \times \prod_{\mathfrak{p} \notin S} U_{\mathfrak{p}} .
$$

It contains $U$ and satisfies

$$
K_{S}=I_{K, S} \cap K^{\times} .
$$

The restricted topology of $I_{K}$ induces the usual direct product topology on $I_{K, S}$. In particular, if $h$ is a homomorphism of $I_{K, S}$ into a finite group $C$ such that $h$ is continuous on $K_{\mathfrak{p}}^{\times}$for each $\mathfrak{p} \in S$ and $h\left(\prod_{\mathfrak{p} \notin S} U_{\mathfrak{p}}\right)=1$, then $h$ is continuous.

Data 1.1. (Basic set) If $K$ is a number field, choose ideles $\alpha_{1}, \ldots, \alpha_{h_{K}}$ which represent $I_{K}$ modulo $U K^{\times}$. Let $S_{0}$ be the set of archimedean primes and those nonarchimedean primes $\mathfrak{p}$ for which $v_{\mathfrak{p}}\left(\alpha_{i, \mathfrak{p}}\right) \neq 0$ for at least one $i$. Then $S_{0}$ is a finite set and each set of primes $S$ which contains $S_{0}$ satisfies

$$
I_{K}=I_{K, S} K^{\times} .
$$

If $K$ is a function field, then we choose $\alpha_{0} \in I_{K}$ of degree 1 and $\alpha_{1}, \ldots, \alpha_{h_{K}}$ which represent $I_{K}^{0}$ modulo $U K^{\times}$. Let $S_{0}$ be the set of primes $\mathfrak{p}$ such that $v_{\mathfrak{p}}\left(\alpha_{i}\right) \neq 0$ for at least one $i$. Again, $S_{0}$ is a finite subset of $\mathbb{P}$ and each set $S_{0} \subseteq S \subseteq \mathbb{P}$ satisfies (1.4). It follows from (1.3) and (1.4) that

$$
C_{K}=I_{K, S} / K_{S}
$$

We increase $S_{0}$ now by adding $\mathbb{P}_{l}$ to it, if $K$ is a number field, and possibly finitely many additional primes which we choose at will. Then we call $S_{0}$ a basic set and fix it for the rest of this work.

Example 1.2. (The cases $K=\mathbb{Q}$ and $K=\mathbb{F}_{q}(t)$ ) For $K=\mathbb{Q}$ the unique factorization in $\mathbb{Z}$ implies that $I_{\mathbb{Q}}=U \mathbb{Q}^{\times}$and therefore $h_{\mathbb{Q}}=1$. We may therefore choose $\alpha_{1}=1$ and $S_{0}$ to be any finite set of primes that contains $\{\infty, l\}$.

For $K=\mathbb{F}_{q}(t)$ we may choose $\alpha_{0}$ as the idele whose component at the pole of $t$ (which we denote by $(t)_{\infty}$ ) is $t^{-1}$ and otherwise is 1 . Again, the unique factorization in $\mathbb{F}_{q}[t]$ implies that $I_{K}^{0}=U K^{\times}$and $h_{K}=1$. We may therefore choose $S_{0}$ to be any finite set of primes of $K$ that contains $(t)_{\infty}$. 


\section{§. Class field theory}

Class field theory teaches us that the idele class group $C_{K}$ controls the abelian extensions of $K$. Specifically, for each finite abelian extension $L / K$ the reciprocity law gives a continuous epimorphism

$$
\psi: C_{K} \longrightarrow \mathcal{G}(L / K)
$$

whose kernel is $N_{L / \bar{K}} C_{L}$ and which is functorial in $L$. For details, we refer the reader to chapters 4 (Serre: local class field theory) and 5 (Tate: global class field theory) of $[\mathrm{CaF}]$ and also to $[\mathrm{Neu}]$ (which however handles only the class field theory of number fields).

For each prime $\mathfrak{p}$ of $K$ we consider $K_{\mathfrak{p}}^{\times}$as the subgroup of $I_{K}$ which consists of all ideles whose $\mathfrak{q}$ coordinate is 1 for each $\mathfrak{q} \neq \mathfrak{p}$. Under this identification $K_{\mathfrak{p}}^{\times} \cap K^{\times}=1$. So, we may and we will consider $K_{\mathfrak{p}}^{\times}$also as a subgroup of $C_{K}$. The restriction of $\psi$ to $K_{\mathfrak{p}}^{\times}$gives a continuous epimorphism

$$
\psi_{\mathfrak{p}}: K_{\mathfrak{p}}^{\times} \longrightarrow D_{\mathfrak{p}}(L / K)
$$

whose kernel is $N_{L_{\mathfrak{p}} / K_{\mathfrak{p}}} L_{\mathfrak{p}}^{\times}$. Here, $L_{\mathfrak{p}}$ is the completion of $L$ with respect to a prime $\mathfrak{p}^{\prime}$ of $L$ which lies over $\mathfrak{p}$ and $D_{\mathfrak{p}}(L / K)$ is the decomposition group of $\mathfrak{p}$ in $L$. Since $L / K$ is abelian, both the norm group and the decomposition group do not depend on $\mathfrak{p}^{\prime}$. The fixed field of $D_{\mathfrak{p}}(L / K)$ is $L \cap K_{\mathfrak{p}}$. It is the maximal subfield of $L / K$ in which $\mathfrak{p}$ completely decomposes. In particular $\mathfrak{p}$ completely decomposes in $L$ if and only if $D_{\mathfrak{p}}(L / K)=1$. The homomorphism $\psi_{\mathfrak{p}}$ maps $U_{\mathfrak{p}}$ onto the inertia group $I_{\mathfrak{p}}(L / K)$ of $L / K$ whose fixed field is the maximal subfield of $L / K$ in which $\mathfrak{p}$ is unramified. Thus $I_{\mathfrak{p}}(L / K)=1$ if and only if $\mathfrak{p}$ is unramified in $L$. If $\varphi$ is unramified at $\mathfrak{p}$, then $\psi_{\mathfrak{p}}\left(\pi_{\mathfrak{p}}\right)=\left(\frac{L / K}{\mathfrak{p}}\right)$ is the Frobenius automorphism of $L / K$ at $\mathfrak{p}$. Finally, the condition $D_{\mathfrak{p}}(L / K)=I_{\mathfrak{p}}(L / K)$ is equivalent for nonarchimedean $\mathfrak{p}$ to $\bar{L}_{\mathfrak{p}}=\bar{K}_{\mathfrak{p}}$.

Let $\tilde{K}$ (resp., $K_{s}$ ) be the algebraic (resp., separable) closure of $K$ and let $G(K)=\mathcal{G}\left(K_{s} / K\right)$ be the absolute Galois group of $K$. We embed $\tilde{K}$ into $\tilde{K}_{\mathfrak{p}}$, thereby extending $\mathfrak{p}$ to $\tilde{K}$. Then $\tilde{K} \cap K_{\mathfrak{p}}=K_{\mathfrak{p} \text {,alg }}$ is a Henselian closure (resp., real closure) of $K$ with respect to $\mathfrak{p}$ if $\mathfrak{p}$ is nonarchimedean (resp., archimedean). Its absolute Galois group $G\left(K_{\mathfrak{p}, \text { alg }}\right)$ is the absolute decomposition group of $\mathfrak{p}$. We denote it by $D_{\mathfrak{p}}$. By Krasner's lemma, $K_{s} K_{\mathfrak{p}}=K_{\mathfrak{p}, s}$. Hence, $\operatorname{res}_{K_{s}}: G\left(K_{\mathfrak{p}}\right) \rightarrow D_{\mathfrak{p}}$ is an isomorphism. We identify $G\left(K_{\mathfrak{p}}\right)$ with $D_{\mathfrak{p}}$ under this map. We denote the maximal unramified 
extension of $K_{\mathfrak{p}}$ by $K_{\mathfrak{p} \text {,ur }}$ (if $\mathfrak{p}$ is archimedean, we set $K_{\mathfrak{p} \text {,ur }}=K_{\mathfrak{p}}$ ) and let $I_{\mathfrak{p}}=\operatorname{res}_{K_{s}}\left(G\left(K_{\mathfrak{p}, \mathrm{ur}}\right)\right)$. This is the absolute inertia group of $\mathfrak{p}$. If $\mathfrak{p}$ is nonarchimedean, then the quotient group $D_{\mathfrak{p}} / I_{\mathfrak{p}} \cong \mathcal{G}\left(K_{\mathfrak{p}, \text { ur }} / K_{\mathfrak{p}}\right)$ is isomorphic to $\hat{\mathbb{Z}}$ and the Frobenius automorphism Frob $_{\mathfrak{p}}$ is a canonical generator of this group.

Whenever we consider a homomorphism $\varphi: G(K) \rightarrow G$ into a finite (not necessarily abelian) group $G$, we assume that $\varphi$ is continuous. Then, the fixed field $L$ of $\operatorname{Ker}(\varphi)$ in $K_{s}$ is a finite extension of $K$. The prime $\mathfrak{p}$ totally decomposes in $L$ if and only if $\varphi\left(D_{\mathfrak{p}}\right)=1$, i.e., $L \subseteq K_{\mathfrak{p}}$. We then say that $\varphi$ totally decomposes at $\mathfrak{p}$. Similarly, we say that $\varphi$ is unramified at $\mathfrak{p}$ if $\mathfrak{p}$ is unramified in $L$, that is, if $\varphi\left(I_{\mathfrak{p}}\right)=1$. In this case $\varphi$ induces a homomorphism of $\mathcal{G}\left(K_{\mathfrak{p} \text {,ur }}\right)$ onto $\mathcal{G}(L / K)$ which maps Frob onto the Frobenius element $\left[\frac{L / K}{\mathfrak{p}^{\prime}}\right]$ of $\mathcal{G}(L / K)$, where $\mathfrak{p}^{\prime}$ is the prime of $L$ which is determined by the embedding of $L$ into $\tilde{K}_{\mathfrak{p}}$. If $\mathfrak{p}$ is nonarchimedean and $\left|I_{\mathfrak{p}}\right|$ is relatively prime to $\operatorname{char}\left(\bar{K}_{\mathfrak{p}}\right)$, then $\varphi$ is tamely ramified at $\mathfrak{p}$. We denote the finite set of primes at which $\varphi$ ramifies by $\operatorname{Ram}(\varphi)$ and also by $\operatorname{Ram}(L / K)$. Finally, $\varphi\left(I_{\mathfrak{p}}\right)=\varphi\left(D_{\mathfrak{p}}\right)$ if and only if $\bar{L}_{\mathfrak{p}}=\bar{K}_{\mathfrak{p}}$. Note that all these concepts are independent of the embedding of $\tilde{K}$ into $\tilde{K}_{\mathfrak{p}}$.

We choose a multiplicative copy $C_{l}$ of the cyclic group of order $l$. Let Hom be the functor of continuous homomorphisms. Then the reciprocity law gives a commutative diagram

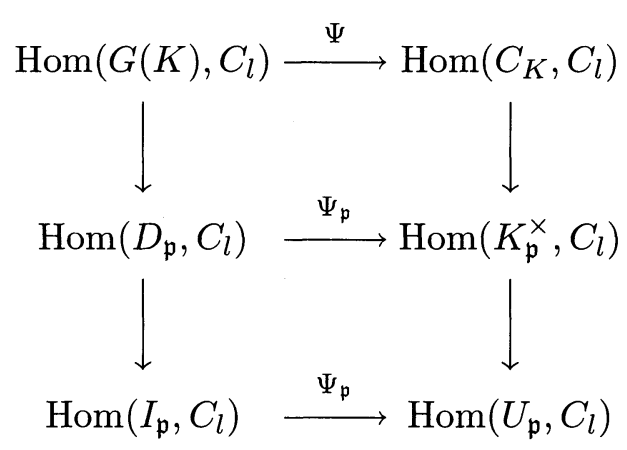

in which the horizontal maps are isomorphisms and the vertical maps are the natural restriction maps. Both $\Psi$ and $\Psi_{\mathfrak{p}}$ map epimorphisms onto epimorphisms. If $h \in \operatorname{Hom}\left(D_{\mathfrak{p}}, C_{l}\right)$ is trivial on $I_{\mathfrak{p}}$ and $\eta=\Psi_{\mathfrak{p}}(h)$, then $\eta$ is trivial on $U_{\mathfrak{p}}$. In this case $h$ induces a homomorphism $\bar{h}: \mathcal{G}\left(K_{\mathfrak{p}, \text { ur }} / K_{\mathfrak{p}}\right) \rightarrow C_{l}$ such that $\bar{h}\left(\right.$ Frob $\left._{\mathfrak{p}}\right)=\eta\left(\pi_{\mathfrak{p}}\right)$. 


\section{§3. Embedding problems; Scholz extensions}

An $l$-group is a group $G$ whose order is a power of $l$. Such a group has a central sequence all of its factors are of order $l$. We consider central embedding problems for $G(K)$ of the following type:

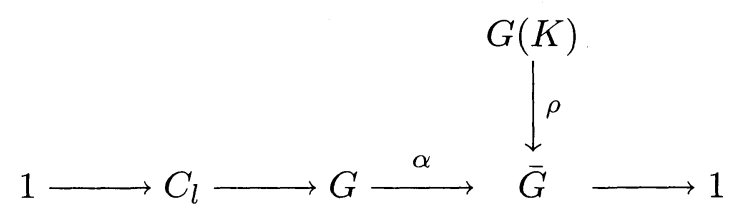

Here the lower sequence is exact, $\rho$ is surjective, and $C_{l}$ is contained in the center of $G$. A weak solution to (3.1) is a homomorphism $\varphi: G(K) \rightarrow G$ such that $\alpha \circ \varphi=\rho$. If $\varphi$ is in addition surjective, we call $\varphi$ a solution. In this case let $L$ (resp., $L^{\prime}$ ) be the fixed field in $K_{s}$ of $\operatorname{Ker}(\rho)$ (resp., $\operatorname{Ker}(\varphi))$. Then $\rho$ (resp., $\varphi$ ) induces an isomorphism $\bar{\rho}: \mathcal{G}(L / K) \rightarrow \bar{G}$ (resp., $\left.\bar{\varphi}: \mathcal{G}\left(L^{\prime} / K\right) \rightarrow G\right)$ such that $\bar{\rho} \circ \operatorname{res}_{L}=\alpha \circ \bar{\varphi}$. The embedding problem splits if the short exact sequence splits. This is the case if and only if $G \cong C_{l} \times \bar{G}$ and $\alpha$ is the projection onto the second factor. Each epimorphism from $G(K)$ onto $C_{l}$ can then be multiplied with $\rho$ to yield a solution to (3.1) and each solution of (3.1) is of this type. In the general case, if $\varphi$ is a weak solution to (3.1), then $C_{l} \cdot \varphi(G(K))=G$ and hence the index of $\varphi(G(K))$ in $G$ divides $l$. Since $G$ is an $l$-group, $\varphi(G(K))$ is normal in $G$. Hence, if (3.1) does not split, then $C_{l} \leq \varphi(G(K))$ and therefore $\varphi$ is a solution to (3.1). In this case, if $\eta: G(K) \rightarrow C_{l}$ is a homomorphism, than the map $\varphi^{\prime}: G(K) \rightarrow G$ defined by $\varphi^{\prime}(\sigma)=\eta(\sigma) \varphi(\sigma)$ for $\sigma \in G(K)$ is also a solution to (3.1). Moreover, each solution to (3.1) is obtained in this way. We write $\varphi^{\prime}=\eta \cdot \varphi$.

For each prime $\mathfrak{p}$ of $K,(3.1)$ gives rise to a local embedding problem:

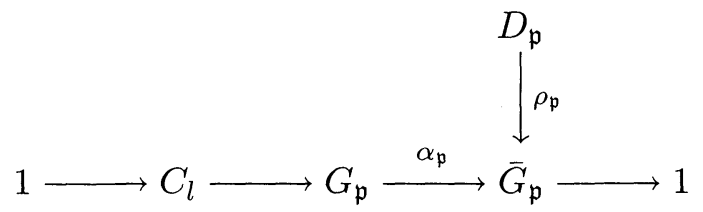

Here $D_{\mathfrak{p}}$ is the absolute decomposition group of $\mathfrak{p}$ (Section 2), $\rho_{\mathfrak{p}}=\left.\rho\right|_{D_{\mathfrak{p}}}$, $\bar{G}_{\mathfrak{p}}=\rho\left(D_{\mathfrak{p}}\right), G_{\mathfrak{p}}=\alpha^{-1}\left(\bar{G}_{\mathfrak{p}}\right)$ and $\alpha_{\mathfrak{p}}=\left.\alpha\right|_{G_{\mathfrak{p}}}$. If (3.1) is central, then so is (3.2). However, even if (3.1) does not split, (3.2) may split. 
Class field theory reduces the solvability of the global embedding problem (3.1) to the solvability of all local embedding problems induced by (3.1) (as we shall see in the proof of Lemma 4.3). This led Scholz to try to construct the map $\rho: G(K) \rightarrow \bar{G}$ with conditions that will impose the solvability of all local embedding problems (3.2). Before we reformulate Scholz's conditions we fix some 'local data' which we would like to impose on $\varphi$.

Local data 3.1. A set $\left\{\varphi_{\mathfrak{p}}: D_{\mathfrak{p}} \rightarrow G \mid \mathfrak{p} \in S_{0}\right\}$ of homomorphisms is a local data for an $l$-group $G$ and a positive integer $n$ if it satisfies the following conditions:

(3.3a) $\varphi_{\mathfrak{p}}\left(I_{\mathfrak{p}}\right)=1$ for each $\mathfrak{p} \in \mathbb{P}_{l}$,

$(3.3 \mathrm{~b})$ if $\mathfrak{p} \in S_{0}$ and $\varphi_{\mathfrak{p}}\left(I_{\mathfrak{p}}\right) \neq 1$, then $\zeta_{l^{n}} \in K_{\mathfrak{p}}$ and $\varphi_{\mathfrak{p}}\left(I_{\mathfrak{p}}\right)=\varphi_{\mathfrak{p}}\left(D_{\mathfrak{p}}\right)$.

We fix the local data for $G$ for the rest of this work.

Our definition of a Scholz extension depends on a finite set $S_{1}$ of $\operatorname{rank}_{l}(K)+\operatorname{rank}_{\infty}(K)$ 'exceptional primes' of $K$ which is disjoint from $S_{0}$. We choose such a set in $\S 5$. It depends on $K$ and on $l$ but not on $G$.

DEFINITION 3.2. (Scholz extension) Let $n$ be a positive integer and let $\varphi$ be an epimorphism of $G(K)$ onto an $l$-group $G$ equipped with a local data as in Local data 3.1. We say that $\varphi$ is $n$-Scholz if

(3.4a) $\zeta_{l^{n}} \in K_{\mathfrak{p}}$, for each $\mathfrak{p} \in \operatorname{Ram}(\varphi)$,

(3.4b) $\varphi\left(I_{\mathfrak{p}}\right)=\varphi\left(D_{\mathfrak{p}}\right)$ for each $\mathfrak{p} \in \operatorname{Ram}(\varphi)$, and

(3.4c) $\left.\varphi\right|_{D_{\mathfrak{p}}}=\varphi_{\mathfrak{p}}$ for each $\mathfrak{p} \in S_{0}$ (Thus, $\varphi$ respects the local data.)

$(3.4 \mathrm{~d}) \varphi\left(I_{\mathfrak{p}}\right)=\varphi\left(D_{\mathfrak{p}}\right)$ for each $\mathfrak{p} \in S_{1}$.

We say that a finite Galois extension $L / K$ is an $l$-extension, if $\mathcal{G}(L / K)$ is an $l$-group. In this case $L / K$ is $n$-Scholz if $\operatorname{res}_{L}: G(K) \rightarrow \mathcal{G}(L / K)$ is an $n$-Scholz epimorphism.

By (3.3a), $\varphi$ ramifies at no $\mathfrak{p} \in \mathbb{P}_{l}$. In other words, if $\mathfrak{p} \in \operatorname{Ram}(\varphi)$, then $\varphi$ is tamely ramified at $\mathfrak{p}$. By $(3.3 \mathrm{~b}), \varphi$ is unramified at each archimedean prime if $l^{n} \neq 2$. Condition (3.4a) then means that $\mathfrak{p}$ totally decomposes in $K\left(\zeta_{l^{n}}\right)$. By Hensel's lemma, it is also equivalent to $\zeta_{l^{n}} \in \bar{K}_{\mathfrak{p}}$ and also 
to $N \mathfrak{p} \equiv 1 \bmod l^{n}$ (Recall that $N \mathfrak{p}$ is the cardinality of $\bar{K}_{\mathfrak{p}}$ ). Condition (3.4b) means that the inertia group of each prime of $L$ which ramifies over $K$ coincides with its decomposition group. If $\varphi_{\mathfrak{p}}=1$ for some $\mathfrak{p} \in S_{0}$, then Condition (3.4c) means that $\mathfrak{p}$ totally decomposes in $L$. If on the other hand, $\varphi_{\mathfrak{p}}\left(I_{\mathfrak{p}}\right) \neq 1$, then Condition (3.4c) implies that $\mathfrak{p} \in \operatorname{Ram}(\varphi)$. Thus, $\operatorname{Ram}(\varphi) \cap S_{0}$ is a priori determined by the local data. Note that all conditions are independent of the particular embedding we have chosen for $\tilde{K}$ into $\tilde{K}_{\mathfrak{p}}$.

In the notation of (3.1), the local data for $G$ induces a local data for $\bar{G}$. This is the set $\left\{\rho_{\mathfrak{p}}=\alpha \circ \varphi_{\mathfrak{p}} \mid \mathfrak{p} \in S_{0}\right\}$. (Note that Condition (3.3) is satified for the $\rho_{\mathfrak{p}}$ 's.) If $\varphi$ is a solution for the embedding problem (3.1) and $\varphi$ is $n$-Scholz, then $\rho$ is $n$-Scholz. But even if $\rho$ is $n$-Scholz, $\varphi$ need not be $n$-Scholz itself. So, in order to continue the induction on the order of $G$, we multiply $\varphi$ by an appropriate homomorphism $\eta: G(K) \rightarrow C_{l}$ such that it will be $n$-Scholz. We do it in two steps. First we change $\varphi$ in this way such that $\operatorname{Ram}(\varphi) \cup S_{1}=\operatorname{Ram}(\rho) \cup S_{1}$. Then we change the resulting $\varphi$ such that $\operatorname{Ram}(\varphi) \cup S_{0} \cup S_{1}=\operatorname{Ram}(\rho) \cup\{\mathfrak{q}\} \cup S_{0} \cup S_{1}$ where $\mathfrak{q}$ is an additional new ramified prime which arises from an application of the Chebotarev density theorem. At this step we use the assumption $\zeta_{l} \notin K$. Thus, if the order of $G$ is $l^{n}$, then we finally realize $G$ as the Galois group of a Galois extension $L / K$ with $\left|\operatorname{Ram}(L / K) \backslash\left(S_{0} \cup S_{1}\right)\right|=n$.

Note the difference between the roles of the finite sets $S_{0}$ and $S_{1}$. The set $S_{0}$ must contain some basic primes but otherwise we are free to make it arbitrarily large and we are completely free to determine $\left.\varphi\right|_{D_{\mathfrak{p}}}$ for each $\mathfrak{p} \in S_{0}$. In particular, we can assume that $\varphi\left(I_{\mathfrak{p}}\right)=1$ for each $\mathfrak{p} \in S_{0}$ and then $\varphi$ will be unramified at each $\mathfrak{p} \in S_{0}$. The behaviour of $\varphi$ at $\mathfrak{p} \in S_{1}$ on the other hand is out of our control. In particular we can not determine whether or not $\varphi$ is ramified at a given $\mathfrak{p} \in S_{1}$. However, $\left|S_{1}\right|=\operatorname{rank}_{l}(K)+\operatorname{rank}_{\infty}(K)$ does not depend on $G$.

\section{§4. Existence of a solution}

The first step toward an $n$-Scholz solution of embedding problem (3.1) is to find a solution which need not be $n$-Scholz. The easier case is when (3.1) splits. In this case (Lemma 4.2) we need to make no assumptions on $\rho$. In the more difficult case (Lemma 4.3) we have to assume that $\rho$ is an $n$-Scholz epimorphism. 
LEMma 4.1. Let $\mathfrak{q}$ be a prime of $K$ which does not belong to $\mathbb{P}_{l} \cup \mathbb{P}_{\infty}$. Suppose that $\zeta_{l} \in K_{\mathfrak{q}}$. Then $U_{\mathfrak{q}} / U_{\mathfrak{q}}^{l} \cong C_{l}$.

Proof. We have assumed in the function field case that $l \neq \operatorname{char}(K)$. Hence, in both cases $l \neq \operatorname{char}\left(\bar{K}_{\mathfrak{q}}\right)$. Hence $\zeta_{l} \in \bar{K}_{\mathfrak{q}}$. By Hensel's Lemma $U_{\mathfrak{q}, 1} \leq U_{\mathfrak{q}}^{l}$. Hence $U_{\mathfrak{q}} / U_{\mathfrak{q}}^{l} \cong \bar{K}_{\mathfrak{q}}^{\times} /\left(\bar{K}_{\mathfrak{q}}^{\times}\right)^{l} \cong C_{l}$.

LEMmA 4.2. Suppose that embedding problem (3.1) splits. Then it has a solution.

Proof. Denote the fixed field of $\operatorname{Ker}(\rho)$ by $L$. Then assume without loss that $\bar{G}=\mathcal{G}(L / K), G=C_{l} \times \mathcal{G}(L / K), \rho=\operatorname{res}_{L}$, and $\alpha$ is the projection on the second factor.

As $K$ is Hilbertian [FrJ, Cor. 12.8], every finite abelian group is realizable over $K$ [FrJ, Thm. 24.48]. In particular suppose that $C_{l}^{m}$ is the maximal $l$-elementary abelian quotient of $\mathcal{G}(L / K)$. Let $N$ be a Galois extension of $K$ with $\mathcal{G}(N / K) \cong C_{l}^{m+1}$. Then $N$ has a subfield $M$ which is linearly disjoint from $L$ over $K$ and $\mathcal{G}(M / K) \cong C_{l}$. So, $L^{\prime}=L M$ satisfies $\mathcal{G}\left(L^{\prime} / K\right) \cong \mathcal{G}(M / K) \times \mathcal{G}(L / K)$ and $\operatorname{res}_{L^{\prime}}: G(K) \rightarrow \mathcal{G}\left(L^{\prime} / K\right)$ gives a solution to (3.1).

Alternatively, let $S=\operatorname{Ram}(L / K) \cup S_{0} \cup S_{1}$. Choose generators $a_{1}, \ldots, a_{s}$ for $K_{S}$ modulo $K_{S}^{l}$ and consider the Galois extension $N=K\left(\zeta_{l}, \sqrt[l]{a_{1}}, \ldots\right.$, $\sqrt[l]{a_{s}}$ ) of $K$. Apply the Chebotarev density theorem to choose a prime $\mathfrak{q} \notin S$ such that $\left(\frac{N / K}{\mathfrak{q}}\right)=1$, i.e., $N \subset K_{\mathfrak{q}}$. In particular, $v_{\mathfrak{q}}\left(a_{i}\right)=0$ and $a_{i} \in U_{\mathfrak{q}}^{l}$, $i=1, \ldots, s$. Also, $\mathfrak{q} \nmid l$. Hence, by Lemma 4.1 , there exists a continuous epimorphism $h_{\mathfrak{q}}: U_{\mathfrak{q}} \rightarrow C_{l}$. Define a continuous homomorphism

$$
h: I_{K, S}=\prod_{\mathfrak{p} \in S} K_{\mathfrak{p}}^{\times} \times U_{\mathfrak{q}} \times \prod_{\mathfrak{p} \notin S \cup\{\mathfrak{q}\}} U_{\mathfrak{p}} \longrightarrow C_{l}
$$

by $h\left(K_{\mathfrak{p}}^{\times}\right)=1$ for $\mathfrak{p} \in S,\left.h\right|_{U_{\mathfrak{q}}}=h_{\mathfrak{q}}$, and $h\left(U_{\mathfrak{p}}\right)=1$ for $\mathfrak{p} \notin S \cup\{\mathfrak{q}\}$. Since $a_{i} \in U_{\mathfrak{q}}^{l}, i=1, \ldots, s, h\left(K_{S}\right)=1$. Hence, $h$ induces an epimorphism $\bar{h}: C_{K} \cong I_{K, S} / K_{S} \rightarrow C_{l}$ which satisfies $\bar{h}\left(K_{\mathfrak{p}}^{\times}\right)=1$ for $\mathfrak{p} \in S, \bar{h}\left(U_{\mathfrak{q}}\right)=C_{l}$, and $h\left(U_{\mathfrak{p}}\right)=1$ for $\mathfrak{p} \notin S \cup\{\mathfrak{q}\}$.

The reciprocity law (2.3) transfers $\bar{h}$ to an epimorphism $\eta: G(K) \rightarrow C_{l}$ such that $\eta\left(I_{\mathfrak{q}}\right)=C_{l}$. Let $M$ be the fixed field of $\operatorname{Ker}(\eta)$ in $K_{s}$. Then $\mathcal{G}(M / K) \cong C_{l}$ and $\mathfrak{q}$ is ramified in $M$. Since $\mathfrak{q}$ is unramified in $L$, we have $M \cap L=K$. Conclude the proof as in the second paragraph. 
The following local global principle is implicit in [Rei], [Ko, p. 35], and [Se, Lemma 2.1.5]. We include a proof of this principle for the convenience of the reader.

LEMMA 4.3. If each of the embedding problems (3.2) has a weak solution, then the embedding problem (3.1) has a solution.

Proof. By lemma 4.2 we may assume that (3.1) does not split. So, by the discussion in the first paragraph of $\S 3$, we have to prove that (3.1) has a weak solution.

To this end let $K^{\prime}=K\left(\zeta_{l}\right)$ and apply class field theory to write a commutative diagram of cohomology groups:

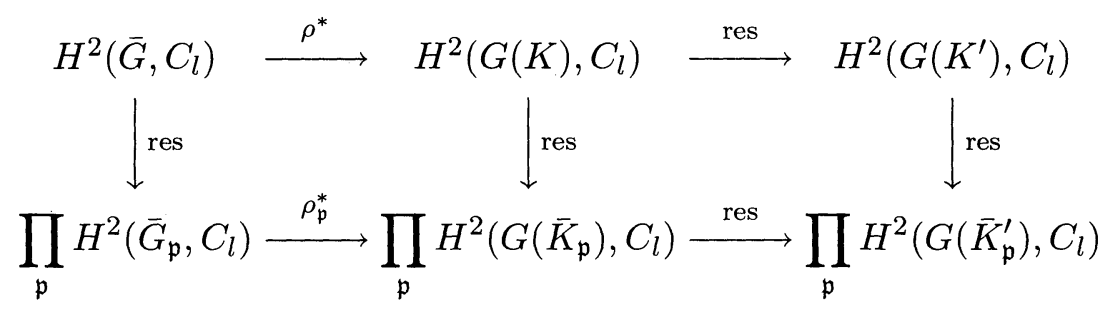

The Brauer-Hasse-Noether theorem for Brauer groups [CaF, p. 185] implies that the right vertical map in (4.1) is injective. Since $\left[K^{\prime}: K\right]$ is relatively prime to $l$, the upper res map in (4.1) is injective [CaF, p. 105]. Hence, the middle vertical map in (4.1) is injective.

Denote now the element of $H^{2}\left(\bar{G}, C_{l}\right)$ (resp., $H^{2}\left(\bar{G}_{\mathfrak{p}}, C_{l}\right)$ ) which corresponds to the short exact sequence by $\varepsilon$ (resp., $\varepsilon_{\mathfrak{p}}$ ). A necessary and sufficient condition for (3.1) (resp., (3.2)) to be weakly solvable is that $\rho^{*}(\varepsilon)=1$ (resp., $\rho_{\mathfrak{p}}^{*}\left(\varepsilon_{\mathfrak{p}}\right)=1$ ) [Hoe, Lemma 1.1] (Note that $C_{l}$ is a multiplicative group).

It follows from the preceding paragraph and from the weak solvability of each of the local embedding problems that (3.1) is weakly solvable.

LEMma 4.4. Let $L / K$ be an $n$-Scholz extension with $\bar{G}=\mathcal{G}(L / K)$. Suppose that the central embedding problem (3.1) does not split and that the exponent of $G$ is at most $l^{n}$. Then (3.1) has a solution.

Proof. By Lemma 4.3, it suffices to prove that each of the local embedding problems (3.2) that (3.1) induces is solvable. To this end we put $L_{\mathfrak{p}}=L K_{\mathfrak{p}}$, replace $\bar{G}_{\mathfrak{p}}$ by the group $\mathcal{G}\left(L_{\mathfrak{p}} / K_{\mathfrak{p}}\right), D_{\mathfrak{p}}$ by $G\left(K_{\mathfrak{p}}\right)$, and $\rho_{\mathfrak{p}}$ by $\operatorname{res}_{L_{\mathfrak{p}}}$. 
If $L_{\mathfrak{p}} / K_{\mathfrak{p}}$ is an unramified extension, then $\rho_{\mathfrak{p}}$ decomposes through a map $\bar{\rho}_{\mathfrak{p}}: \hat{\mathbb{Z}}=\mathcal{G}\left(K_{\mathfrak{p}, \text { ur }} / K_{\mathfrak{p}}\right) \rightarrow \mathcal{G}\left(L_{\mathfrak{p}} / K_{\mathfrak{p}}\right)$ and therefore $(3.2)$ is weakly solvable.

If $L_{\mathfrak{p}} / K_{\mathfrak{p}}$ is ramified, then by (3.4b) and (3.4a), it is totally ramified and $\zeta_{l^{n}} \in K_{\mathfrak{p}}$. By (3.3a) $l \neq \operatorname{char}\left(\bar{K}_{\mathfrak{p}}\right)$ and therefore the ramification is tame. Hence $L_{\mathfrak{p}}$ is a cyclic Kummer extension. Since $\mathcal{G}\left(L_{\mathfrak{p}} / K_{\mathfrak{p}}\right)$ is isomorphic to a subgroup of $\bar{G}$ and since the exponent of $\bar{G}$ is at most $l^{n-1}$, the order of $\mathcal{G}\left(L_{\mathfrak{p}} / K_{\mathfrak{p}}\right)$ is at most $l^{n-1}$. Thus $L_{\mathfrak{p}}=K_{\mathfrak{p}}\left(\sqrt[\sqrt[m]{-1}^{a}]{a}\right)$ for some $m \leq n$ and $a \in K_{\mathfrak{p}}$ with $v_{\mathfrak{p}}(a)=0$ [CaF, p. 32]. If (3.2) splits, then it certainly has a weak solution. If (3.2) does not split, then $G_{\mathfrak{p}}$ is a cyclic group of order $l^{m}$. Then $L_{\mathfrak{p}}^{\prime}=K_{\mathfrak{p}}\left(\sqrt[l^{m}]{a}\right)$ is a cyclic extension of $K_{\mathfrak{p}}$ of degree $l^{m}$ which contains $L_{\mathfrak{p}}$. The composition of res: $G\left(K_{\mathfrak{p}}\right) \rightarrow \mathcal{G}\left(L_{\mathfrak{p}}^{\prime} / K_{\mathfrak{p}}\right)$ with an isomorphism $\mathcal{G}\left(L_{\mathfrak{p}}^{\prime} / K_{\mathfrak{p}}\right) \rightarrow G_{\mathfrak{p}}$ which maps generators of both groups to the same generator of $\mathcal{G}\left(L_{\mathfrak{p}} / K_{\mathfrak{p}}\right)$ solves the local embedding problem (3.2).

\section{$\S 5$. Linearly disjoint fields; an exceptional set of primes}

The condition that Lemma 5.1 imposes on the subgroup $A$ of $\mathbb{F}_{l}^{\times}$to be nontrivial is translated in Lemma 5.2 to $\zeta_{l} \notin K$. This condition restricts the Scholz-Reichardt method of realizing $l$-groups over $K$ to the case where $\zeta_{l} \notin K$. In particular, as $\zeta_{2}=-1$ belongs to every field, the method fails for $l=2$.

The introduction of an 'exceptional set of primes' (Definition 5.4, Lemma 5.5, and Data 5.6) allow us to handle number fields and the case $l \mid h_{K}$. To find such a set and also for further applications we construct a special Galois extension $N^{*}$ of $K$. Then we use the Chebotarev density theorem and find primes of $K$ whose Artin symbol is the conjugacy class of a given element of $\mathcal{G}\left(N^{*} / K\right)$ and therefore have specific decomposition behaviour in $N^{*}$.

LEMMA 5.1. Let $A$ be a nontrivial subgroup of $\mathbb{F}_{l}^{\times}$which acts on the direct product $C=C_{l}^{m}$ in a natural way: $\left(c_{1}, \ldots, c_{m}\right)^{\alpha}=\left(c_{1}^{\alpha}, \ldots, c_{m}^{\alpha}\right)$. Then the semidirect product $G=C \rtimes A$ has no nontrivial quotients of order $l$.

Proof. Let $h: G \rightarrow C_{l}$ be a homomorphism. Then the order of $h(A)$ divides both $l$ and $l-1$, and therefore $h(A)=1$. Let now $1 \neq \alpha \in A$ and $c \in C$. Then $h(c)^{\alpha}=h\left(c^{\alpha}\right)=h\left(\alpha^{-1} c \alpha\right)=h(\alpha)^{-1} h(c) h(\alpha)=h(c)$ and hence $h(c)=1$. Conclude that $h=1$. 
Let $S$ be a finite set of primes of $K$ and consider elements $a_{1}, \ldots, a_{s}$ of $K_{S}$. We say that $a_{1}, \ldots, a_{s}$ are multiplicatively independent modulo $K_{S}^{l}$ if each relation

$$
a_{1}^{l_{1}} \cdots a_{s}^{l_{s}}=b^{l}
$$

with $l_{1}, \ldots, l_{s} \in \mathbb{Z}$ and $b \in K_{S}$ implies that $l \mid l_{i}, i=1, \ldots, s$. Replace ' $b \in$ $K_{S}$ ' by ' $b \in K^{\times}$' to define the expression multiplicatively independent modulo $\left(K^{\times}\right)^{l}$.

LEMMA 5.2. Let $S$ be a finite set of primes of $K$. Let $a_{1}, \ldots, a_{s}$ be multiplicatively independent elements of $K_{S}$ modulo $K_{S}^{l}$. Then the fields $K\left(\zeta_{l}, \sqrt[l]{a_{1}}\right), \ldots, K\left(\zeta_{l}, \sqrt[l]{a_{s}}\right)$ are linearly disjoint and of degree $l$ over $K\left(\zeta_{l}\right)$.

Further, suppose that $\zeta_{l} \notin K$. Let $L$ be an l-extension of $K$ and let $n$ be a positive integer. Then, the fields $L\left(\zeta_{l^{n}}, \sqrt[l]{a_{1}}\right), \ldots, L\left(\zeta_{l^{n}}, \sqrt[l]{a_{s}}\right)$ are linearly disjoint and of degree $l$ over $L\left(\zeta_{l^{n}}\right)$. In particular, $\left[L\left(\zeta_{l^{n}}, \sqrt[l]{a_{1}}, \ldots, \sqrt[l]{a_{s}}\right)\right.$ : $\left.L\left(\zeta_{l^{n}}\right)\right]=l^{s}$.

Proof. Observe that $a_{1}, \ldots, a_{s}$ are even multiplicatively independent modulo $\left(K^{\times}\right)^{l}$. Indeed, let $l_{1}, \ldots, l_{s} \in \mathbb{Z}$ and $b \in K^{\times}$such that (5.1) holds. Then $l v_{\mathfrak{p}}(b)=0$ and therefore $v_{\mathfrak{p}}(b)=0$ for each $\mathfrak{p} \notin S$. So, $b \in K_{S}$. Hence $l$ divides $l_{1}, \ldots, l_{s}$, as desired.

It follows that $a_{1}, \ldots, a_{s}$ are also multiplicatively independent modulo $\left(K\left(\zeta_{l}\right)^{\times}\right)^{l}$. Indeed, $k=\left[K\left(\zeta_{l}\right): K\right]$ divides $l-1$ and is therefore relatively prime to $l$. If in (5.1) $b \in K\left(\zeta_{l}\right)$, we take the norm of both sides to obtain

$$
a_{1}^{k l_{1}} \cdots a_{s}^{k l_{s}}=\left(N_{K\left(\zeta_{l}\right) / K^{b}}\right)^{l} .
$$

By the preceding paragraph $l \mid k l_{i}$. Hence $l \mid l_{i}$ for $i=1 \ldots, s$, as desired.

By Kummer theory [Lan, p. 220, Thm. 14], the fields $K\left(\zeta_{l}, \sqrt[l]{a_{1}}\right), \ldots$, $K\left(\zeta_{l}, \sqrt[l]{a_{s}}\right)$ are linearly disjoint and of degree $l$ over $K\left(\zeta_{l}\right)$. Hence $M=$ $K\left(\zeta_{l}, \sqrt[l]{a_{1}}, \ldots, \sqrt[l]{a_{s}}\right)$ is a Galois extension of $K$ and $\mathcal{G}(M / K)$ is the semidirect product of $\mathcal{G}\left(M / K\left(\sqrt[l]{a_{1}}, \ldots, \sqrt[l]{a_{s}}\right)\right)$ with $\mathcal{G}\left(M / K\left(\zeta_{l}\right)\right) \cong \mathbb{F}_{l}^{s}$. The former group is isomorphic to $\mathcal{G}\left(K\left(\zeta_{l}\right) / K\right)$ and therefore to a subgroup of $\mathbb{F}_{l}^{\times}$which acts on the latter group by scalar multiplication. Indeed, if $\sigma \in \mathcal{G}\left(M / K\left(\sqrt[l]{a_{1}}, \ldots, \sqrt[l]{a_{s}}\right)\right)$ satisfies $\zeta_{l}^{\sigma}=\zeta_{l}^{s}$ for some $s \in \mathbb{F}_{l}^{\times}$and $\tau \in$ $\mathcal{G}\left(M / K\left(\zeta_{l}\right)\right)$, then $\sigma^{-1} \tau \sigma=\tau^{s}$.

We may write $K\left(\zeta_{l^{n}}\right)=K\left(\zeta_{l}\right) K^{\prime}$ where $K^{\prime}$ is a cyclic extension of $K$ of degree $l^{m}$ with $m \leq n-1$. So, $L K^{\prime}$ is an $l$-extension of $K$. Since, by 
assumption, $\mathcal{G}\left(K\left(\zeta_{l}\right) / K\right)$ is nontrivial, $M / K$ has no Galois subextension of degree $l$ (Lemma 5.1). Hence $M \cap L K^{\prime}=K$ and therefore $M \cap L\left(\zeta_{l^{n}}\right)=$ $K\left(\zeta_{l}\right)$. Let $N=L\left(\zeta_{l^{n}}, \sqrt[l]{a_{1}}, \ldots, \sqrt[l]{a_{s}}\right)$. Then $\mathcal{G}\left(N / L\left(\zeta_{l^{n}}\right)\right) \cong \mathcal{G}\left(M / K\left(\zeta_{l}\right)\right)$. In particular $L\left(\zeta_{l^{n}}, \sqrt[l]{a_{1}}\right), \ldots, L\left(\zeta_{l^{n}}, \sqrt[l]{a_{s}}\right)$ are linearly disjoint and of degree $l$ over $L\left(\zeta_{l^{n}}\right)$.

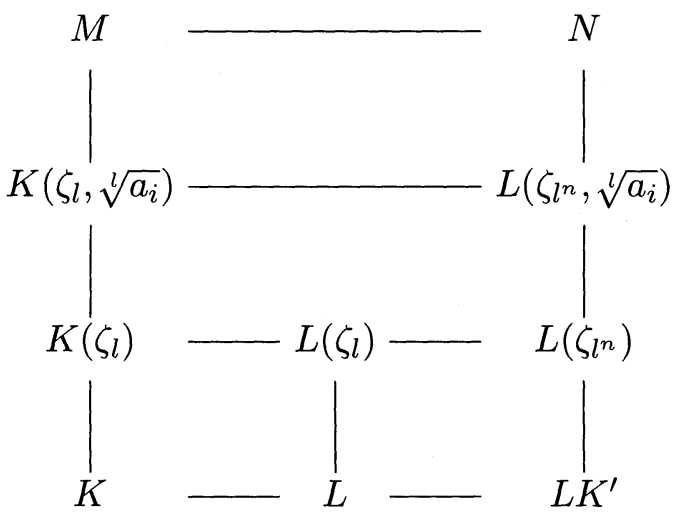

Remark 5.3. Under the assumption of Lemma 5.2 suppose that $L$ is a function field over a finite field $K_{0}$. Then $N=L\left(\zeta_{l^{n}}, \sqrt[l]{a_{1}}, \ldots, \sqrt[l]{a_{s}}\right)$ is a regular extension of $K_{0}\left(\zeta_{l^{n}}\right)$

Indeed, let $L\left(\zeta_{l^{\infty}}\right)=L\left(\zeta_{l}, \zeta_{l^{2}}, \zeta_{l^{3}}, \ldots\right)$. Since $n$ is arbitrary in the last paragraph of the proof of Lemma 5.2, we have that $M \cap L\left(\zeta_{l^{\infty}}\right)=K\left(\zeta_{l}\right)$. Since $K_{0}\left(\zeta_{l \infty}\right)$ has no $l$-extensions and $M / K\left(\zeta_{l}\right)$ is an $l$-extension, this implies that $M \cap L \tilde{K}_{0}=K\left(\zeta_{l}\right)$. Hence $N \cap L \tilde{K}_{0}=L\left(\zeta_{l^{n}}\right)$. Since $L / K_{0}$ is regular, $L\left(\zeta_{l^{n}}\right) \cap \tilde{K}_{0}=K_{0}\left(\zeta_{l^{n}}\right)$. Conclude that $N \cap \tilde{K}_{0}=K_{0}\left(\zeta_{l^{n}}\right)$, that is $N / K_{0}\left(\zeta_{l^{n}}\right)$ is regular.

DEFINITION 5.4. (Exceptional set of primes) Let $r=\operatorname{rank}_{l}(K)$ and choose $\alpha_{1}, \ldots, \alpha_{r}$ in $I_{K}$ (resp., $I_{K}^{0}$ ) which represent a multiplicative basis over $\mathbb{F}_{l}$ for $\mathrm{Cl}(K)_{l}$ (resp., $\mathrm{Cl}_{0}(K)_{l}$ ) if $K$ is a number field (resp., function field). By definition there exist $\mu_{i} \in U$ and $a_{i}^{*} \in K^{\times}$such that

$$
\alpha_{i}^{l}=\mu_{i} a_{i}^{*}, \quad i=1, \ldots, r .
$$

We call $a_{1}^{*}, \ldots, a_{r}^{*}$ an $l$-basis for $K$ and fix it for the whole work. We also choose fundamental units of $K$. These are elements $w_{1}, \ldots, w_{s} \in E_{K}$, with $s=\operatorname{rank}_{\infty}(K)$, which generate $E_{K}$ modulo $\mu_{K}$. We fix them too for the whole work. We also fix the following basic Galois extension of $K$ :

$$
N^{*}=K\left(\zeta_{l^{n}}, \sqrt[l]{a_{1}^{*}}, \ldots, \sqrt[l]{a_{r}^{*}}, \sqrt[l]{w_{1}}, \ldots, \sqrt[l]{w_{s}}\right)
$$


By (5.2), $\operatorname{Ram}\left(N^{*} / K\right) \subseteq \mathbb{P}_{l} \cup \mathbb{P}_{\infty}$.

An $n$-exceptional set of primes of $K$ is a set of finite primes

$$
S_{1}=\left\{\mathfrak{p}_{1}^{*}, \ldots, \mathfrak{p}_{r}^{*}, \mathfrak{q}_{1}^{*}, \ldots, \mathfrak{q}_{s}^{*}\right\}
$$

which is disjoint from $S_{0}$ such that for all $i, i^{\prime} \in\{1, \ldots, r\}$ and all $j, j^{\prime} \in$ $\{1, \ldots, s\}$ we have

$$
\begin{aligned}
& a_{i}^{*} \in K_{\mathfrak{p}_{i^{\prime}}^{*}}^{l} \Longleftrightarrow i \neq i^{\prime} ; \quad a_{i}^{*} \in K_{\mathfrak{q}_{j}^{*}}^{l}, \\
& w_{j} \in K_{\mathfrak{p}_{i}^{*}}^{l} ; \quad w_{j} \in K_{\mathfrak{q}_{j^{\prime}}^{*}}^{l} \Longleftrightarrow j \neq j^{\prime}, \text { and } \\
& \zeta_{l^{n}} \in K_{\mathfrak{p}_{i}^{*}}, \zeta_{l^{n}} \in K_{\mathfrak{q}_{j}^{*}} .
\end{aligned}
$$

LEMMA 5.5. (Characterization of exceptional set) Let

$$
\begin{array}{r}
N_{i}=K\left(\zeta_{l^{n}}, \sqrt[l]{a_{1}^{*}}, \ldots, \sqrt[l]{a_{i-1}^{*}}, \sqrt[l]{a_{i+1}^{*}}, \ldots, \sqrt[l]{a_{r}^{*}}, \sqrt[l]{w_{1}}, \ldots, \sqrt[l]{w_{s}}\right) \\
i=1, \ldots, r \\
N_{j}^{\prime}=K\left(\zeta_{l^{n}}, \sqrt[l]{a_{1}^{*}}, \ldots, \sqrt[l]{a_{r}^{*}}, \sqrt[l]{w_{1}}, \ldots, \sqrt[l]{w_{j-1}}, \sqrt[l]{w_{j+1}}, \ldots, \sqrt[l]{w_{s}}\right), \\
j=1, \ldots, s
\end{array}
$$

Then

(a) $N^{*} / N_{i}$ and $N^{*} / N_{j}^{\prime}$ are cyclic extensions of degree $l, N^{*} / K$ is a $G a$ lois extension of degree $\left[K\left(\zeta_{l}^{n}\right): K\right] l^{r+s}, N_{i} / K$ and $N_{j}^{\prime} / K$ are Galois extensions, and

(b) the set $S_{1}$ (of (5.3)) is exceptional if and only if it is disjoint from $S_{0}$, and

$$
D_{\mathfrak{p}_{i}^{*}}\left(N^{*} / K\right)=\mathcal{G}\left(N^{*} / N_{i}\right) \text { and } D_{\mathfrak{q}_{j}^{*}}\left(N^{*} / K\right)=\mathcal{G}\left(N^{*} / N_{j}^{\prime}\right),
$$

for $i=1, \ldots, r$ and $j=1, \ldots, s$.

Proof. Let $S=\mathbb{P}_{\infty}$. Once we prove that $a_{1}^{*}, \ldots, a_{r}^{*}, w_{1}, \ldots, w_{s}$ are multiplicatively independent modulo $K^{\times}$, (a) will follow from Lemma 5.2 with $L=K$. Since the fixed field of the decomposition group of a prime $\mathfrak{p}$ in $N^{*}$ is $N^{*} \cap K_{\mathfrak{p}}$, statement (b) will then also hold. Note that since $\mathcal{G}\left(N^{*} / N_{i}\right)$ and $\mathcal{G}\left(N^{*} / N_{j}^{\prime}\right)$ are normal subgroups of $\mathcal{G}\left(N^{*} / K\right),(5.5)$ is independent of the embedding we have chosen for $\tilde{K}$ in $\tilde{K}_{\mathfrak{p}_{i}^{*}}$. 
Suppose therefore that $k_{1}, \ldots, k_{r}, l_{1}, \ldots, l_{s}$ are integers and $b \in K^{\times}$ such that

$$
\left(a_{1}^{*}\right)^{k_{1}} \cdots\left(a_{r}^{*}\right)^{k_{r}} w_{1}^{l_{1}} \cdots w_{s}^{l_{s}}=b^{l}
$$

Then, by (5.2),

$$
\mu_{1}^{-k_{1}} \cdots \mu_{r}^{-k_{r}} w_{1}^{l_{1}} \cdots w_{s}^{l_{s}}=\left(\alpha_{1}^{-k_{1}} \cdots \alpha_{r}^{-k_{r}} b\right)^{l} .
$$

Since the left hand side of (5.7) belongs to $U$, so is its right hand side and therefore $\alpha_{1}^{k_{1}} \cdots \alpha_{r}^{k_{r}} b^{-1} \in U$. Hence $\alpha_{1}^{k_{1}} \cdots \alpha_{r}^{k_{r}} \in U K^{\times}$. By the choice of $\alpha_{1}, \ldots, \alpha_{r}$ each $k_{i}$ is a multiple of $l$.

It follows from (5.6) that there exists $c \in K^{\times}$such that $w_{1}^{l_{1}} \cdots w_{s}^{l_{s}}=c^{l}$. Hence $c \in E_{K}$ and since $w_{1}, \ldots, w_{s}$ are multiplicatively independent over $\mathbb{Z}$ and generate $E_{K}$ modulo $\mu_{K}$, each $l_{j}$ is a multiple of $l$. Conclude that $a_{1}^{*} \ldots, a_{r}^{*}, w_{1}, \ldots, w_{s}$ are multiplicatively independent modulo $\left(K^{\times}\right)^{l}$.

Data 5.6. (Exceptional set of primes) Choose a generator $\sigma_{i}^{*}$ for $\mathcal{G}\left(N^{*} /\right.$ $\left.N_{i}\right)$, let $\operatorname{Con}\left(\sigma_{i}^{*}\right)$ be the conjugacy class of $\sigma_{i}^{*}$ in $\mathcal{G}\left(N^{*} / K\right)$ and apply the Chebotarev density theorem to choose $\mathfrak{p}_{i}^{*} \in \mathbb{P} \backslash S_{0}$ such that $\left(\frac{N^{*} / K}{\mathfrak{p}_{i}^{*}}\right)=$ $\operatorname{Con}\left(\sigma_{i}^{*}\right), i=1, \ldots, r$. Similarly, choose a generator $\tau_{j}^{*}$ for $\mathcal{G}\left(N^{*} / N_{j}^{\prime}\right)$ and $\mathfrak{q}_{j}^{*} \in \mathbb{P} \backslash S_{0}$ such that $\left(\frac{N^{*} / K}{\mathfrak{q}_{j}^{*}}\right)=\operatorname{Con}\left(\tau_{j}^{*}\right), j=1, \ldots, s$. Since the Artin symbol of a prime generates its decomposition group in $N^{*},(5.5)$ holds and therefore, by Lemma 5.5 , the set $S_{1}$ of (5.3), chosen in this way is exceptional. We fix it for the rest of this work and note that $\left|S_{1}\right|=\operatorname{rank}_{\infty}(K)+\operatorname{rank}_{l}(K)$.

Note that if $K$ is a number field, then $S_{1}$ is empty exactly if either $K=$ $\mathbb{Q}$ and $l \neq 2, K=\mathbb{Q}(\sqrt{-3})$ and $l \neq 2,3$, or $K$ is another imaginary quadratic field with $l \neq 2$ and $l \nmid h_{K}$. If $K$ is a function field, then $\operatorname{rank}_{\infty}(K)=0$ and $\left|S_{1}\right|=\operatorname{rank}_{l}(K)$. In this case $S_{1}=\emptyset$ is equivalent to $l \nmid h_{K}$.

\section{$\S 6$. Getting rid of extra ramification}

The second step in the solution of embedding problem (3.1) is to change the solution $\psi_{0}$ we have found in Lemma 4.4 such that in addition to the primes of $\operatorname{Ram}(\rho)$ the only primes of $K$ at which the solution ramifies belong to the exceptional set $S_{1}$ which we have chosen in Data 5.6.

LEMMA 6.1. Let $S$ be a finite set of primes which is disjoint from the exceptional set $S_{1}$. For each prime $\mathfrak{p} \in S$ let $h_{\mathfrak{p}}: U_{\mathfrak{p}} \rightarrow C_{l}$ be a continuous 
homomorphism. Suppose that $\zeta_{l} \notin K$. Then there exists a continuous homomorphism $h: C_{K} \rightarrow C_{l}$ such that $\left.h\right|_{U_{\mathfrak{p}}}=h_{\mathfrak{p}}$ for each $\mathfrak{p} \in S$ and $h\left(U_{\mathfrak{p}}\right)=$ 1 for each prime $\mathfrak{p} \notin S \cup S_{1}$

Proof. We break the proof into five parts.

Part A. (Definition of $h_{\mathfrak{p}}$ for $\mathfrak{p} \in S_{1}$ )

By (5.4), $\zeta_{l} \in U_{\mathfrak{p}}$. Hence, by Lemma 4.1, $U_{\mathfrak{p}} / U_{\mathfrak{p}}^{l} \cong C_{l}$. Hence, for each $u \in U_{\mathfrak{p}} \backslash U_{\mathfrak{p}}^{l}$ and each $c \in C_{l}$ there exists a continuous homomorphism $h^{\prime}: U_{\mathfrak{p}} \rightarrow C_{l}$ such that $h^{\prime}(u)=c$.

By (5.2) and (5.4), $\mu_{i} \in U_{\mathfrak{p}_{i}^{*}} \backslash U_{\mathfrak{p}_{i}^{*}}^{l}$. Hence, there exists a continuous homomorphism $h_{\mathfrak{p}_{i}^{*}}: U_{\mathfrak{p}_{2}^{*}} \rightarrow C_{l}$ such that

$$
\left(\prod_{\mathfrak{p} \in S} h_{\mathfrak{p}}\left(\mu_{i, \mathfrak{p}}\right)\right) \cdot h_{\mathfrak{p}_{\imath}^{*}}\left(\mu_{i, \mathfrak{p}_{i}^{*}}\right)=1, \quad i=1, \ldots, r .
$$

Here, $\mu_{i, \mathfrak{p}}$ is the $\mathfrak{p}$-th component of $\mu_{i}$. For the same reason, there exists a continuous homomorphism $h_{\mathfrak{q}_{j}^{*}}: U_{\mathfrak{q}_{j}^{*}} \rightarrow C_{l}$ such that

$$
\left(\prod_{\mathfrak{p} \in S} h_{\mathfrak{p}}\left(w_{j}\right)\right) \cdot h_{\mathfrak{q}_{j}^{*}}\left(w_{j}\right)=1, \quad j=1, \ldots, s
$$

This completes the definition of $h_{\mathfrak{p}}$ for each $\mathfrak{p} \in S_{1}$.

By (5.2) and (5.4), $\mu_{i} \in U_{\mathfrak{p}}^{l}$ and therefore

$$
h_{\mathfrak{p}}\left(\mu_{i, \mathfrak{p}}\right)=1 \text { for each } \mathfrak{p} \in S_{1} \backslash\left\{\mathfrak{p}_{i}^{*}\right\}
$$

By (5.4), $w_{j} \in U_{\mathfrak{p}}^{l}$ and therefore

$$
h_{\mathfrak{p}}\left(w_{j}\right)=1 \text { for each } \mathfrak{p} \in S_{1} \backslash\left\{\mathfrak{q}_{j}^{*}\right\}
$$

Part B. (Definition of $f^{\prime}$ on $U K^{\times} / K^{\times}$)

The formula

$$
f(\mu)=\prod_{\mathfrak{p} \in S \cup S_{1}} h_{\mathfrak{p}}\left(\mu_{\mathfrak{p}}\right)
$$

defines a continuous homomorphism $f$ from the open subgroup $U$ of $I_{K}$ into $C_{l}$ that is trivial on $U_{\mathfrak{p}}$ for each $\mathfrak{p} \notin S \cup S_{1}$ and coincides with $h_{\mathfrak{p}}$ on $U_{\mathfrak{p}}$ for each $\mathfrak{p} \in S \cup S_{1}$. By (6.2) and (6.4), $f\left(w_{j}\right)=1$ for $j=1, \ldots, s$. Since $\left|\mu_{K}\right|$ is relatively prime to $l, f$ is trivial on $\mu_{K}$. It follows that $f$ is trivial on $E_{K}=U \cap K^{\times}$. So, $f$ defines a homomorphism $\bar{f}: U / E_{K} \rightarrow C_{l}$ which we compose with the isomorphism $U K^{\times} / K^{\times} \cong U / E_{K}$ to get a continuous homomorphism $f^{\prime}: U K^{\times} / K^{\times} \rightarrow C_{l}$. 
Part C. (Claim: $\left.I_{K}^{l} K^{\times} \cap U K^{\times}=U^{l} K^{\times} \cdot \prod_{i=1}^{r}\left\langle\mu_{i}\right\rangle\right)$

Indeed, as $\mu_{i}=\alpha_{i}^{l}\left(a_{i}^{*}\right)^{-1}$, by (5.2), the right hand side is contained in the left hand side. Each element of the left hand side has the form $\xi=\alpha^{l} a=\mu b$ with $\alpha \in I_{K}, a, b \in K^{\times}$, and $\mu \in U$. Thus $\alpha^{l}=\mu b a^{-1} \in$ $U K^{\times}$. If $K$ is a function field, then $l \operatorname{deg}(\alpha)=\operatorname{deg}(\mu)+\operatorname{deg}\left(b a^{-1}\right)=0$ and hence $\operatorname{deg}(\alpha)=0$, so that $\alpha \in I_{K}^{0}$. So, in any case $\alpha=\nu c \prod_{i=1}^{r} \alpha_{i}^{k_{i}}$ for some $\nu \in U, c \in K^{\times}$, and $k_{i} \in \mathbb{Z}, i=1, \ldots, r$. It follows from (5.2) that $\xi=\nu^{l}\left(c^{l} \prod_{i=1}^{r}\left(a_{i}^{*}\right)^{k_{i}}\right) \prod_{i=1}^{r} \mu_{i}^{k_{i}}$ belongs to the right hand side. This concludes the proof of the claim.

Part D. (Definition of $g$ on $I_{K}^{l} U K^{\times} / K^{\times}$)

By (6.1) and (6.3), $f\left(\mu_{i}\right)=1, i=1, \ldots, r$. Hence, by Part C, $f^{\prime}$ is trivial on $\left(I_{K}^{l} K^{\times} \cap U K^{\times}\right) / K^{\times}$. So, $f^{\prime}$ extends to a continuous homomorphism $g: I_{K}^{l} U K^{\times} / K^{\times} \rightarrow C_{l}$ which is trivial on $I_{K}^{l} K^{\times} / K^{\times}$, coincides with $h_{\mathfrak{p}}$ on $U_{\mathfrak{p}}$ for each $\mathfrak{p} \in S \cup S_{1}$, and is trivial $U_{\mathfrak{p}}$ for each $\mathfrak{p} \notin S \cap S_{1}$.

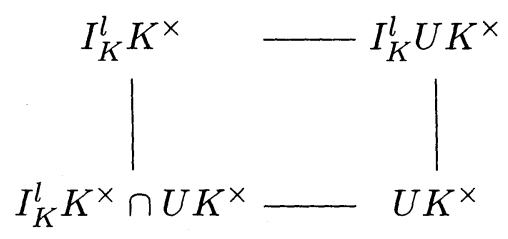

Part E. (Conclusion of the proof)

Finally observe that $I_{K} / U K^{\times}$is a finitely generated abelian profinite group (Section 1). Hence, $I_{K}^{l} U K^{\times} / I_{K}^{l} K^{\times}$is an open subgroup of $C_{K} / C_{K}^{l}$. The latter group may be considered as a vector space over $\mathbb{F}_{l}$ and $I_{K}^{l} U K^{\times} / K^{\times}$has a closed complement in it. So, $g$ extends to a continuous homomorphism $h: C_{K} \rightarrow C_{l}$.

LEMMA 6.2. Suppose that the central embedding problem (3.1) does not split. Assume that $\zeta_{l} \notin K$. If the central embedding problem (3.1) has a solution $\psi_{0}$, then (3.1) also has a solution $\psi: G(K) \rightarrow G$ for which $\operatorname{Ram}(\psi) \subseteq \operatorname{Ram}(\rho) \cup S_{1}$.

Proof. Let $S=\operatorname{Ram}\left(\psi_{0}\right) \backslash\left(S_{1} \cup \operatorname{Ram}(\rho)\right)$. If $\mathfrak{p} \in S$, then $\rho\left(I_{\mathfrak{p}}\right)=1$ and therefore $\psi_{0}\left(I_{\mathfrak{p}}\right) \leq C_{l}$. (Actually, as $\mathfrak{p} \in \operatorname{Ram}\left(\psi_{0}\right)$, we have $\psi_{0}\left(I_{\mathfrak{p}}\right) \neq 1$ and therefore $\psi_{0}\left(I_{\mathfrak{p}}\right)=C_{l}$.) The reciprocity law (2.3) associates with $\left.\psi_{0}\right|_{I_{\mathfrak{p}}}$ a continuous homomorphism $h_{\mathfrak{p}}: U_{\mathfrak{p}} \rightarrow C_{l}$. By Lemma 6.1 there exists a continuous homomorphism $h: C_{K} \rightarrow C_{l}$ such that $\left.h\right|_{U_{\mathfrak{p}}}=h_{\mathfrak{p}}$ for each $\mathfrak{p} \in S$ 
and $h\left(U_{\mathfrak{p}}\right)=1$ for each $\mathfrak{p} \notin S \cup S_{1}$. Apply again the reciprocity law to obtain a continuous homomorphism $\eta: G(K) \rightarrow C_{l}$ such that $\left.\eta\right|_{I_{\mathfrak{p}}}=\left.\psi_{0}\right|_{I_{\mathfrak{p}}}$ for each $\mathfrak{p} \in S$ and $\eta\left(I_{\mathfrak{p}}\right)=1$ for each $\mathfrak{p} \notin S \cup S_{1}$. Consider the solution $\psi=\eta^{-1} \cdot \psi_{0}$ to $(3.1)$. Then, $\psi\left(I_{\mathfrak{p}}\right)=1$ for each $\mathfrak{p} \in S \cup\left[\mathbb{P} \backslash\left(\operatorname{Ram}\left(\psi_{0} \cup S_{1}\right)\right)\right]$. Hence, $\operatorname{Ram}(\psi) \subseteq(\mathbb{P} \backslash S) \cap\left[\operatorname{Ram}\left(\psi_{0}\right) \cup S_{1}\right] \subseteq \operatorname{Ram}(\rho) \cup S_{1}$, as desired.

\section{$\S 7$. Scholz solution of a nonsplitting embedding problem}

The last step in the solution of embedding problem (3.1) is to multiply the solution which Lemma 6.2 gives with a homomorphism $\eta: G(G) \rightarrow C_{l}$ such that the resulting solution will be $n$-Scholz.

LEMMA 7.1. Let $S$ be a finite set of primes of $K$ which contains $S_{0}$. Let $L$ be a finite l-extension of $K$ and let $n$ be a positive integer. For each $\mathfrak{p} \in S$ let $h_{\mathfrak{p}}: K_{\mathfrak{p}}^{\times} \rightarrow C_{l}$ be a homomorphism. Suppose that $\zeta_{l} \notin K$.

Then there exists a prime $\mathfrak{q}$ of $K$ and there exists a continuous homomorphism $h: C_{K} \rightarrow C_{l}$ such that

(a) $\mathfrak{q} \notin S$ and $L\left(\zeta_{l^{n}}\right) \subseteq K_{\mathfrak{q}}$,

(b) $\left.h\right|_{K_{\mathfrak{p}}^{\times}}=h_{\mathfrak{p}}$ for each $\mathfrak{p} \in S$,

(c) $h\left(U_{\mathfrak{q}}\right)=C_{l}$,

(d) $h\left(U_{\mathfrak{p}}\right)=1$ for each $\mathfrak{p} \notin S \cup\{\mathfrak{q}\}$.

Proof. We break the proof into five parts.

Part A. (Reduction of the lemma to constructing a homomorphism $g: \bar{I}_{K, S} /$ $\left.\bar{K}_{S} \rightarrow C_{l}\right)$

Let $\left(K_{S}: K_{S}^{l}\right)=l^{s}$. Choose generators $a_{1}, \ldots, a_{s}$ for $K_{S}$ modulo $K_{S}^{l}$. For each $\mathfrak{q} \notin S$ we can decompose $I_{K, S}$ as

$$
I_{K, S}=\prod_{\mathfrak{p} \in S} K_{\mathfrak{p}}^{\times} \times U_{\mathfrak{q}} \times \prod_{\mathfrak{p} \notin S \cup\{\mathfrak{q}\}} U_{\mathfrak{p}} .
$$

Use a bar to denote the reduction of elements and subgroups of $I_{K, S}$ modulo $I_{K, S}^{l}$. In particular ${ }^{1}$

$$
\bar{I}_{K, S}=\prod_{\mathfrak{p} \in S} \overline{K_{\mathfrak{p}}^{\times}} \times \bar{U}_{\mathfrak{q}} \times \prod_{\mathfrak{p} \notin S \cup\{\mathfrak{q}\}} \bar{U}_{\mathfrak{p}} .
$$

\footnotetext{
${ }^{1}$ Do not confuse $\overline{K_{\mathfrak{p}}^{\times}} \cong K_{\mathfrak{p}}^{\times} /\left(K_{\mathfrak{p}}^{\times}\right)^{l}$ with the multiplicative group of the $\bar{K}_{\mathfrak{p}}^{\times}$of the residue field $\bar{K}_{\mathfrak{p}}$, for $\mathfrak{p} \notin \mathbb{P}_{\infty}$.
} 
and

$$
\bar{K}_{S}=\left\langle\bar{a}_{1}, \ldots, \bar{a}_{s}\right\rangle
$$

Also, $h_{\mathfrak{p}}: K_{\mathfrak{p}}^{\times} \rightarrow C_{l}$ induces a homomorphism $\bar{h}_{\mathfrak{p}}: \overline{K_{\mathfrak{p}}^{\times}} \rightarrow C_{l}$. Then $\bar{I}_{K, S} / \bar{K}_{S}$ $=\left(I_{K, S} / I_{K, S}^{l}\right) /\left(K_{S} I_{K, S}^{l} / I_{K, S}^{l}\right)$ is a quotient of $C_{K}=I_{K, S} / K_{S}$ (See (1.5)). Hence, it suffices to find a prime $\mathfrak{q}$ of $K$ which satisfies (a) and to construct a homomorphism $g: \bar{I}_{K, S} \rightarrow C_{l}$ such that

$$
\begin{aligned}
& \left.g\right|_{K_{\mathfrak{p}}^{\times}}=\bar{h}_{\mathfrak{p}} \quad \text { for each } \mathfrak{p} \in S, \\
& g\left(\bar{U}_{\mathfrak{q}}\right)=C_{l}, \\
& g\left(\bar{U}_{\mathfrak{p}}\right)=1 \quad \text { for each } \mathfrak{p} \notin S \cup\{\mathfrak{q}\} . \\
& g\left(\bar{a}_{i}\right)=1 \quad \text { for } i=1, \ldots, s .
\end{aligned}
$$

By (7.1) and (7.3), $g$ will induce a homomorphism $\bar{g}: \bar{I}_{K, S} / \bar{K}_{S} \rightarrow C_{l}$ which will compose with the canonical homomorphism $C_{K} \rightarrow \bar{I}_{K, S} / \bar{K}_{S}$ to the desired homomorphism $h$.

Part B. (Presentation of $\bar{a}_{i}$ as an idele)

For each $i$ between 1 and $s$ and each $\mathfrak{p}$ let $a_{i \mathfrak{p}}$ be $a_{i}$ considered as an element of $K_{\mathfrak{p}}$ and let

$$
\delta_{i}=\prod_{\mathfrak{p} \in S} h_{\mathfrak{p}}\left(a_{i \mathfrak{p}}\right)
$$

If $\mathfrak{q}$ satisfies (a), then $a_{1}, \ldots, a_{s}, \zeta_{l} \in U_{\mathfrak{q}}$ and $\bar{U}_{\mathfrak{q}} \cong C_{l}$ (Lemma 4.1). Choose a generator $\bar{u}_{\mathfrak{q}}$ of $\bar{U}_{\mathfrak{q}}$. For each $i$ there exists then $0 \leq \beta_{i}<l$ such that $\bar{a}_{i \mathfrak{q}}=\bar{u}_{\mathfrak{q}}^{\beta_{i}}$. The representation of $\bar{a}_{i}$ as an idele will therefore take the form:

$$
\bar{a}_{i}=\prod_{\mathfrak{p} \in S} \bar{a}_{i \mathfrak{p}} \cdot \bar{u}_{\mathfrak{q}}^{\beta_{i}} \cdot \prod_{\mathfrak{p} \notin S \cup\{\mathfrak{p}\}} \bar{a}_{i \mathfrak{p}} .
$$

Conditions (7.3a) and (7.3c) force that $g\left(\bar{a}_{i \mathfrak{p}}\right)=h\left(\bar{a}_{i \mathfrak{p}}\right)$ for $\mathfrak{p} \in S$ and $g\left(\bar{a}_{i \mathfrak{p}}\right)=1$ for $\mathfrak{p} \notin S \cup\{\mathfrak{q}\}$. Condition $(7.3 \mathrm{~b})$ is equivalent to $g\left(\bar{u}_{\mathfrak{q}}\right) \neq 1$. We have therefore to choose $\mathfrak{q}$ such that (a) will hold and to define $g\left(\bar{u}_{\mathfrak{q}}\right)$ as a nonzero element of $C_{l}$ such that (7.3d) will be satisfied.

If $\delta_{i}=1$ for $i=1, \ldots, r$ we may use the Chebotarev density theorem to choose $\mathfrak{q} \notin S$ such that

$$
N=L\left(\zeta_{l^{n}}, \sqrt[l]{a_{1}}, \ldots, \sqrt[l]{a_{s}}\right) \subseteq K_{\mathfrak{q}}
$$


In particular (a) holds and $a_{\imath \mathfrak{q}} \in U_{\mathfrak{q}}^{l}$ so that $\beta_{i}=0$ for $i=1, \ldots, s$. We therefore define $g\left(\bar{u}_{\mathfrak{q}}\right)$ to be a generator of $C_{l}$ and derive from (7.7) that $g\left(\bar{a}_{i}\right)=\delta_{i} \cdot g\left(\bar{u}_{\mathfrak{q}}\right)^{\beta_{\imath}}=1$, so that $(7.3 \mathrm{~d})$ holds.

Part C. (The main case)

We may and we will from now assume that

$$
\delta_{1} \neq 1
$$

Under this assumption there exists $0 \leq \varepsilon_{i}<l$ such that in $C_{l}$

$$
\delta_{1}^{\varepsilon_{2}}=\delta_{i}, \quad i=1,2, \ldots, s .
$$

In particular $\varepsilon_{1}=1$. Define

$$
b_{1}=a_{1} \text { and } b_{i}=a_{i} / a_{1}^{\varepsilon_{i}}, \text { for } i=2, \ldots, s .
$$

As $a_{1}, \ldots, a_{s}$ are multiplicatively independent modulo $K_{S}^{l}$, so are $b_{1}, \ldots, b_{s}$. By Lemma $5.2, L\left(\zeta_{l^{n}}, \sqrt[l]{b_{1}}\right), \ldots, L\left(\zeta_{l^{n}}, \sqrt[l]{b_{s}}\right)$ are linearly disjoint fields of degree $l$ over $L\left(\zeta_{l^{n}}\right)$.

Part D. (Choosing $\mathfrak{q}$ )

Part $\mathrm{C}$ allows us to choose $\sigma \in \mathcal{G}\left(N / L\left(\zeta_{l^{n}}\right)\right)$ with $\sigma \sqrt[l]{a_{1}}=\zeta_{l} \sqrt[l]{a_{1}}$ and $\sigma \sqrt[l]{b_{i}}=\sqrt[l]{b_{\imath}}, i=2, \ldots, s$.

Chebotarev density theorem gives a prime $\mathfrak{q} \notin S$ such that $\left(\frac{N / K}{q}\right)=$ $\operatorname{Con}(\sigma)$. Thus, $L\left(\zeta_{l^{n}}\right) \subseteq K_{\mathfrak{q}}$ but $K_{\mathfrak{q}}\left(\zeta_{l^{n}}, \sqrt[l]{a_{1}}\right)$ is an unramified extension of $K_{\mathfrak{q}}$ of degree $l$. In particular $a_{1} \in U_{\mathfrak{q}} \backslash U_{\mathfrak{q}}^{l}$. On the other hand $b_{i} \in U_{\mathfrak{q}}^{l}$ and therefore, by (7.11)

$$
\bar{a}_{i \mathfrak{q}}=\bar{a}_{1 \mathfrak{q}}^{\varepsilon_{\imath}} \quad i=2, \ldots, s .
$$

\section{Part E. (Definition of $g$ )}

By Part $\mathrm{B}, \bar{a}_{1 \mathfrak{q}}=\bar{u}_{1 \mathfrak{q}}^{\beta}$ with $0<\beta<l$. We may therefore define $g\left(\bar{u}_{\mathfrak{q}}\right)$ as the element of $C_{l}$ that satisfies

$$
g\left(\bar{u}_{\mathfrak{q}}\right)^{\beta}=\delta_{1}^{-1}
$$

In particular $g\left(\bar{u}_{\mathfrak{q}}\right) \neq 1$. By $(7.12), \bar{a}_{i \mathfrak{q}}=\bar{u}_{\mathfrak{q}}^{\beta \varepsilon_{2}}, i=2, \ldots, s$. As $\varepsilon_{1}=1$ the latter equality also holds for $i=1$. This gives (7.7) the following form:

$$
\bar{a}_{i}=\prod_{\mathfrak{p} \in S} \bar{a}_{i \mathfrak{p}} \cdot \bar{u}_{\mathfrak{q}}^{\beta \varepsilon_{\mathfrak{p}}} \prod_{\mathfrak{p} \notin S \cup\{\mathfrak{q}\}} \bar{a}_{i \mathfrak{p}} .
$$


Apply $g$ on (7.14) and use (7.3a), (7.3c), (7.6), (7.13), and (7.10) to get that

$$
g\left(\bar{a}_{i}\right)=\left(\prod_{\mathfrak{p} \in S} g\left(\bar{a}_{i \mathfrak{p}}\right)\right) \cdot g\left(\bar{u}_{\mathfrak{q}}\right)^{\beta \varepsilon_{\mathfrak{\imath}}}=\delta_{i} \delta_{1}^{-\varepsilon_{i}}=1 .
$$

So (7.3d) holds and the proof is complete.

LEMMA 7.2. Suppose that (3.1) is a central embedding problem such that $\rho$ is an $n$-Scholz epimorphism which respects the local data $\left\{\rho_{\mathfrak{p}}=\alpha \circ \varphi_{\mathfrak{p}} \mid\right.$ $\left.\mathfrak{p} \in S_{0}\right\}$. Suppose further that $\zeta_{l} \notin K$ and that (3.1) has a solution $\psi$ such that $\operatorname{Ram}(\psi) \cup S_{1}=\operatorname{Ram}(\rho) \cup S_{1}$. Then there exists a prime $\mathfrak{q} \notin$ $\operatorname{Ram}(\psi) \cup S_{0} \cup S_{1}$ at which $\rho$ totally decomposes and there exists an $n$-Scholz solution $\varphi: G(K) \rightarrow G$ to (3.1) which respects the local data $\left\{\varphi_{\mathfrak{p}} \mid \mathfrak{p} \in S_{0}\right\}$ such that $\operatorname{Ram}(\varphi) \cup S_{0}=\operatorname{Ram}(\psi) \cup\{\mathfrak{q}\} \cup S_{0}$.

Proof. Let $L$ (resp., $L^{\prime}$ ) be the fixed field of $\operatorname{Ker}(\rho)$ (resp., $\operatorname{Ker}(\psi)$ ) in $K_{s}$. For each $\mathfrak{p} \in S=\operatorname{Ram}(\psi) \cup S_{0} \cup S_{1}$ we define a homomorphism $\eta_{\mathfrak{p}}: D_{\mathfrak{p}} \rightarrow C_{l}$ as follows:

If $\mathfrak{p} \in\left(\operatorname{Ram}(\psi) \backslash S_{0}\right) \cup S_{1}$, then $\mathfrak{p} \in \operatorname{Ram}(\rho) \cup S_{1}$. Choose a lifting of Frob $_{\mathfrak{p}}$ to an element $\sigma_{\mathfrak{p}}$ of $D_{\mathfrak{p}}$. Since $\rho$ is $n$-Scholz, we have $\alpha \circ \psi\left(\sigma_{\mathfrak{p}}\right)=$ $\rho\left(\sigma_{\mathfrak{p}}\right) \in \rho\left(D_{\mathfrak{p}}\right)=\rho\left(I_{\mathfrak{p}}\right)=\alpha \circ \psi\left(I_{\mathfrak{p}}\right)$. Hence, there exists $\tau \in I_{\mathfrak{p}}$ and $\gamma_{\mathfrak{p}} \in C_{l}$ such that $\psi\left(\sigma_{\mathfrak{p}}\right)=\gamma_{\mathfrak{p}} \psi(\tau)$. Replace $\sigma_{\mathfrak{p}}$ by $\sigma_{\mathfrak{p}} \tau^{-1}$, if necessary, to assume that $\psi\left(\sigma_{\mathfrak{p}}\right)=\gamma_{\mathfrak{p}}$. Now define a homomorphism $\bar{\eta}_{\mathfrak{p}}$ from $\left\langle\right.$ Frob $\left.\mathrm{p}_{\mathfrak{p}}\right\rangle=D_{\mathfrak{p}} / I_{\mathfrak{p}} \cong$ $\hat{\mathbb{Z}}$ to $C_{l}$ by $\bar{\eta}\left(\right.$ Frob $\left._{\mathfrak{p}}\right)=\gamma_{\mathfrak{p}}^{-1}$. Then compose $\bar{\eta}_{\mathfrak{p}}$ with the canonical map $D_{\mathfrak{p}} \rightarrow D_{\mathfrak{p}} / I_{\mathfrak{p}}$ to a homomorphism $\eta_{\mathfrak{p}}: D_{\mathfrak{p}} \rightarrow C_{l}$. It satisfies

$$
\eta_{\mathfrak{p}}\left(I_{\mathfrak{p}}\right)=1 \text { and } \eta_{\mathfrak{p}}\left(\sigma_{\mathfrak{p}}\right)=\psi\left(\sigma_{\mathfrak{p}}\right)^{-1}
$$

If $\mathfrak{p} \in S_{0}$, then $\left.\alpha \circ \psi\right|_{D_{\mathfrak{p}}}=\rho_{\mathfrak{p}}=\alpha \circ \varphi_{\mathfrak{p}}$. Hence $\varphi_{\mathfrak{p}}=\left.\eta_{\mathfrak{p}} \cdot \psi\right|_{D_{\mathfrak{p}}}$ with a map $\eta_{\mathfrak{p}}: D_{\mathfrak{p}} \rightarrow C_{l}$. Since $C_{l}$ is contained in the center of $G, \eta_{\mathfrak{p}}$ is a homomorphism.

Lemma 7.1, applied to $L^{\prime}$ instead of to $L$ and the reciprocity law (2.3) supply a prime $\mathfrak{q} \in \mathbb{P}$ and a continuous epimorphism $\eta: G(K) \rightarrow C_{l}$ such that

(7.16a) $\mathfrak{q} \notin S$ and $L^{\prime}\left(\zeta_{l^{n}}\right) \subseteq K_{\mathfrak{q}}$; in particular $\psi\left(D_{q}\right)=1$,

(7.16b) $\left.\eta\right|_{D_{\mathfrak{p}}}=\eta_{\mathfrak{p}}$ for each $\mathfrak{p} \in S$,

(7.16c) $\eta\left(I_{\mathfrak{q}}\right)=C_{l}$, and 
(7.16d) $\eta\left(I_{\mathfrak{p}}\right)=1$ for each $\mathfrak{p} \notin S \cup\{\mathfrak{q}\}$.

We prove that $\varphi=\eta \cdot \psi: G(K) \rightarrow G$ satisfies the requirements of the lemma. To this end, let $\mathfrak{p}$ be a prime of $K$. We have to prove that if $\mathfrak{p} \notin S_{0}$, then $\varphi\left(I_{\mathfrak{p}}\right) \neq 1$ if and only if $\psi\left(I_{\mathfrak{p}}\right) \neq 1$ or $\mathfrak{p}=\mathfrak{q}$. In addition, if $\varphi\left(I_{\mathfrak{p}}\right) \neq 1$ or $\mathfrak{p} \in S_{1}$, then $\varphi\left(I_{\mathfrak{p}}\right)=\varphi\left(D_{\mathfrak{p}}\right)$ should hold. Finally, we have to prove that $\left.\varphi\right|_{D_{\mathfrak{p}}}=\varphi_{\mathfrak{p}}$ for each $\mathfrak{p} \in S_{0}$. We distinguish between several cases:

Case A. $\left(\mathfrak{p} \in\left(\operatorname{Ram}(\dot{\psi}) \backslash S_{0}\right) \cup S_{1}\right)$

Then, by $(7.15),\left.\varphi\right|_{I_{\mathfrak{p}}}=\left.\left.\eta\right|_{I_{\mathfrak{p}}} \cdot \psi\right|_{I_{\mathfrak{p}}}=\left.\psi\right|_{I_{\mathfrak{p}}}$. So, $\psi\left(I_{\mathfrak{p}}\right) \neq 1$ if and only if $\varphi\left(I_{\mathfrak{p}}\right) \neq 1$. Also, by (7.15) and (7.16b) $\varphi\left(\sigma_{\mathfrak{p}}\right)=\eta_{\mathfrak{p}}\left(\sigma_{\mathfrak{p}}\right) \psi\left(\sigma_{\mathfrak{p}}\right)=1$. Hence, $\varphi\left(D_{\mathfrak{p}}\right)=\varphi\left(\left\langle\sigma_{\mathfrak{p}}, I_{\mathfrak{p}}\right\rangle\right)=\varphi\left(I_{\mathfrak{p}}\right)$.

Case B. $\left(\mathfrak{p} \in S_{0}\right)$

By $(7.16 \mathrm{~b}),\left.\varphi\right|_{D_{\mathfrak{p}}}=\left.\eta_{\mathfrak{p}} \cdot \psi\right|_{D_{\mathfrak{p}}}=\varphi_{\mathfrak{p}}$. So, $\varphi$ respects the given local data. Case C. $(\mathfrak{p}=\mathfrak{q})$

By $(7.16 \mathrm{a}), \psi\left(D_{\mathfrak{q}}\right)=1$ and hence, by $(7.16 \mathrm{c}), \varphi\left(I_{\mathfrak{q}}\right)=C_{l}$. Also, for each $\sigma \in D_{\mathfrak{q}}$ we have $\varphi(\sigma)=\eta(\sigma) \cdot 1 \in C_{l}$. Hence $C_{l}=\varphi\left(I_{\mathfrak{q}}\right) \leq \varphi\left(D_{\mathfrak{q}}\right) \leq C_{l}$. So, $\varphi\left(I_{\mathfrak{q}}\right)=\varphi\left(D_{\mathfrak{q}}\right)$.

Case D. $(\mathfrak{p} \notin S \cup\{\mathfrak{q}\})$

Then $\left.\varphi\right|_{I_{\mathfrak{p}}}=\left.\left.\eta\right|_{I_{\mathfrak{p}}} \cdot \psi\right|_{I_{\mathfrak{p}}}=1$.

In each case all the requirements are fulfilled.

We combine Lemmas 4.2, 4.3, 6.2, and 7.2:

Proposition 7.3. (Solution of an embedding problem) Let $K$ be a global field and let $l \neq \operatorname{char}(K)$ be a prime such that $\zeta_{l} \notin K$. Let $S_{0}$ be a basic set of primes (Data 1.1), let $n$ be a positive integer, and let $S_{1}$ be an $n$-exeptional set of primes (Data 5.6). Consider an embedding problem (3.1) for $G(K)$ for which $G$ is an l-group of exponent $l^{n}$. Let $\left\{\varphi_{\mathfrak{p}} \mid \mathfrak{p} \in S_{0}\right\}$ be a local data for $G$ and $n$. Suppose that $\rho$ is an $n$-Scholz epimorphism which respects the local data $\left\{\alpha \circ \varphi_{\mathfrak{p}} \mid \mathfrak{p} \in S_{0}\right\}$. Then there exists a prime $\mathfrak{q} \notin \operatorname{Ram}(\rho) \cup S_{0} \cup S_{1}$ and there exists an $n$-Scholz solution $\varphi$ for (3.1) which respects the local data $\left\{\varphi_{\mathfrak{p}} \mid \mathfrak{p} \in S_{0}\right\}$ such that

$$
\operatorname{Ram}(\varphi) \cup S_{0} \cup S_{1}=\operatorname{Ram}(\rho) \cup\{\mathfrak{q}\} \cup S_{0} \cup S_{1}
$$

THEOREM 7.4. (Realization of $l$-groups) Let $K$ be a global field and let $l \neq \operatorname{char}(K)$ be a prime such that $\zeta_{l} \notin K$. Let $S_{0}$ be a basic set of 
primes (Data 1.1), let $m \leq n$ be positive integers, and let $S_{1}$ be an $n$ exeptional set of primes (Data 5.6). Consider a group $G$ of order $l^{m}$ and a local data $\left\{\varphi_{\mathfrak{p}}: D_{\mathfrak{p}} \rightarrow G \mid \mathfrak{p} \in S_{0}\right\}$ for $G$. Then $K$ has a finite Galois extension $L$ which is $n$-Scholz such that $\mathcal{G}(L / K) \cong G$, for each $\mathfrak{p} \in S_{0}$ the map res: $D_{\mathfrak{p}} \rightarrow D_{\mathfrak{p}}(L / K)$ coincides with $\varphi_{\mathfrak{p}}$, and there exist $m$ primes $\mathfrak{q}_{1}, \ldots, \mathfrak{q}_{m} \in \mathbb{P} \backslash\left(S_{0} \cup S_{1}\right)$ such that

$$
\operatorname{Ram}(L / K) \cup S_{0} \cup S_{1}=\left\{\mathfrak{q}_{1}, \ldots, \mathfrak{q}_{m}\right\} \cup S_{0} \bullet S_{1}
$$

In particular, if each $\mathfrak{p} \in S_{0}$ completely decomposes in $L$, then

$$
m \leq|\operatorname{Ram}(L / K)| \leq m+\operatorname{rank}_{l}(K)+\operatorname{rank}_{\infty}(K) .
$$

Proof. Suppose without loss that $m \geq 1$ and embed $C_{l}$ in the center of $G$, let $\bar{G}=G / C_{l}$, and let $\alpha: G \rightarrow \bar{G}$ be the canonical map. Induction on the order of the group gives an $n$-Scholz epimorphism $\rho: G(K) \rightarrow \bar{G}$ which respects the local data $\left\{\alpha \circ \varphi_{\mathfrak{p}} \mid \mathfrak{p} \in S_{0}\right\}$ such that $\left|\operatorname{Ram}(\rho) \backslash\left(S_{0} \cup S_{1}\right)\right|=$ $m-1$. This creates an embedding problem (3.1). Proposition 7.3 supplies an $n$-Scholz solution of this problem such that (7.17) holds. Conclude that (7.18) is true. As $\left|S_{1}\right|=\operatorname{rank}_{l}(K)+\operatorname{rank}_{\infty}(K)$, this gives the estimates (7.19).

Example 7.5. (Necessity of many ramified primes) We prove in this example that if $L / K$ is an l-elementary abelian extension, then $\operatorname{Ram}(L / K)$ must be 'big'. More precisely, we compute a constant $r_{0}$ such that if $[L: K]=l^{r}$ and $l$ is unramified in $L$, then $|\operatorname{Ram}(L / K)| \geq r-r_{0}$.

Indeed, let $T$ be a finite set of $m$ primes which is disjoint from $\mathbb{P}_{l} \cup \mathbb{P}_{\infty}$ and let $S=\mathbb{P}_{l} \cup \mathbb{P}_{\infty} \cup T$. Let $r$ be the maximal integer for which $K$ admits a Galois extension $N$ which is unramified outside $S$ such that $\mathcal{G}(N / K) \cong C_{l}^{r}$. Class field theory suggests a bound on $r$ which does not depend on $T$.

ClaIM. The following inequalities hold for a number field $K$ :

$$
r \leq[K: \mathbb{Q}]+\left|\mathbb{P}_{l}\right|+\operatorname{rank}_{l}(K)+m, \text { if } l \neq 2
$$

(7.20b) $r \leq[K: \mathbb{Q}]+\left|\mathbb{P}_{2}\right|+\#$ real archimedean primes $+\operatorname{rank}_{2}(K)+m$, if $l=2$

(7.20c) $r \leq 1+m$, if $K=\mathbb{Q}$ and $l \neq 2$,

(7.20d) $r=2+m$, if $K=\mathbb{Q}$ and $l=2$, 
The following inequalities hold for a function field $K$ :

(7.20e) $r \leq 1+\operatorname{rank}_{l}(K)+m$, if $K$ is a function field,

(7.20f) $r \leq 1+m$, if $K=\mathbb{F}_{q}(t)$.

Indeed, let $V=\prod_{\mathfrak{p} \notin S} U_{\mathfrak{p}}$. By the reciprocity law (2.3), $r$ is the maximal integer for which there exists a homomorphism $h: I_{K} \rightarrow C_{l}^{r}$ which is trivial on $V K^{\times}$. As $V \leq U$ (in the notation of Section 1), we have the following short exact sequence

$$
1 \longrightarrow U K^{\times} / V K^{\times} \longrightarrow I_{K} / V K^{\times} \longrightarrow \mathrm{Cl}(K) \longrightarrow 1 .
$$

Observe that $U K^{\times} / V K^{\times}$is a homomorphic image of $U / V \cong \prod_{\mathfrak{p} \in S} U_{\mathfrak{p}}$. Hence

$$
\begin{aligned}
r & =\operatorname{dim}_{\mathbb{F}_{l}}\left(I_{K} / V K^{\times}\right) /\left(I_{K} / V K^{\times}\right)^{l} \\
& \leq \operatorname{dim}_{\mathbb{F}_{l}}\left(U K^{\times} / V K^{\times}\right) /\left(U K^{\times} / V K^{\times}\right)^{l}+\operatorname{dim}_{\mathbb{F}_{l}} \operatorname{Cl}(K) / \mathrm{Cl}(K)^{l} \\
& \leq \sum_{\mathfrak{p} \in S} \operatorname{dim}_{\mathbb{F}_{l}} U_{\mathfrak{p}} / U_{\mathfrak{p}}^{l}+\operatorname{dim}_{\mathbb{F}_{l}} \mathrm{Cl}(K) / \mathrm{Cl}(K)^{l} .
\end{aligned}
$$

For each $\mathfrak{p} \in \mathbb{P}_{l}$ the dimension of $U_{\mathfrak{p}} / U_{\mathfrak{p}}^{l}$ over $\mathbb{F}_{l}$ is $\left[K_{\mathfrak{p}}: \mathbb{Q}_{l}\right]+1$ if $\zeta_{l} \in K_{\mathfrak{p}}$ and $\left[K_{\mathfrak{p}}: \mathbb{Q}_{l}\right]$ if $\zeta_{l} \notin K_{\mathfrak{p}}$. If $\mathfrak{p} \in \mathbb{P}_{\infty}$, then $U_{\mathfrak{p}}=U_{\mathfrak{p}}^{l}$ unless $l=2$ and $\mathfrak{p}$ is real. In the latter case $U_{\mathfrak{p}}=\mathbb{R}^{\times}$and so $\operatorname{dim}_{\mathbb{F}_{2}} U_{\mathfrak{p}} / U_{\mathfrak{p}}^{2}=1$. If $\mathfrak{p} \notin \mathbb{P}_{l} \cup \mathbb{P}_{\infty}$, then $U_{\mathfrak{p}}=U_{\mathfrak{p}}^{l}$ unless $\zeta_{l} \in U_{\mathfrak{p}}$, in which case $\operatorname{dim}_{\mathbb{F}_{l}} U_{\mathfrak{p}} / U_{\mathfrak{p}}^{l}=1$. Thus

$$
\begin{aligned}
r \leq \sum_{\mathfrak{p} \in \mathbb{P}_{l}}\left(\left[K_{\mathfrak{p}}: \mathbb{Q}_{l}\right]+1\right) & +\left(\sum_{\mathfrak{p} \text { real }} 1\right) \\
& +m+\operatorname{dim}_{\mathbb{F}_{l}} \mathrm{Cl}(K) / \mathrm{Cl}(K)^{l}
\end{aligned}
$$

$$
\begin{aligned}
\leq[K: \mathbb{Q}]+\left|\mathbb{P}_{l}\right|+\text { \# } & \text { real archimedean primes } \\
& +\operatorname{dim}_{\mathbb{F}_{l}} \mathrm{Cl}(K) / \mathrm{Cl}(K)^{l}+m
\end{aligned}
$$

where the term '\#real archimedean primes' appears only if $l=2$.

If $K$ is a number field, then $\operatorname{dim}_{\mathbb{F}_{l}} \mathrm{Cl}(K) / \mathrm{Cl}(K)^{l}=\operatorname{rank}_{l}(K)$. If $l \neq 2$, then we can drop $\sum_{\mathfrak{p} \text { real }} 1$ from (7.22b). So, (7.20a) and (7.20b) hold.

If $K=\mathbb{Q}$, then $\mathrm{Cl}(K)=1$ and therefore $\operatorname{dim}_{\mathbb{F}_{l}} \mathrm{Cl}(K) / \mathrm{Cl}(K)^{l}=0$. If in addition, $l \neq 2$, that $\zeta_{l} \notin \mathbb{Q}_{l}$ and therefore $\mathbb{P}_{l}=\{l\}$ contributes 1 to the $\operatorname{sum} \sum_{\mathfrak{p} \in S} \operatorname{dim}_{\mathbb{F}_{l}} U_{\mathfrak{p}} / U_{\mathfrak{p}}^{l}$. If instead, $l=2$, then \#real archimedean primes $=1$. This gives $(7.20 \mathrm{c})$ and $(7.20 \mathrm{~d})$. 
If $K$ is a function field, then $\left|\mathbb{P}_{l}\right|=\left|\mathbb{P}_{\infty}\right|=0$ but

$$
\operatorname{dim}_{\mathbb{F}_{l}} \mathrm{Cl}(K) / \mathrm{Cl}(K)^{l}=\operatorname{rank}_{l}(K)+1
$$

This gives (7.20e). Finally, if $K=\mathbb{F}_{q}(t)$, then $\operatorname{rank}_{l}(K)=0$ and so (7.20f) is true.

Thus, in each case, there exists a constant $r_{0}$ that does not depend on $m$ such that $r \leq r_{0}+m$. If $L / K$ is a Galois extension with $\mathcal{G}(L / K) \cong C_{l}^{r}$ and we take $T=\operatorname{Ram}(L / K) \backslash \mathbb{P}_{l}$, then we find that $r-r_{0} \leq|T|$, which is somewhat stronger than claimed.

Consider now the case in which $l \neq 2$ and $K=\mathbb{Q}$. Then $r_{0}=1$ and $\mathbb{Q}$ has a unique extension $L_{0}$ of degree $l$ in which $l$ ramifies. It is the unique extension of degree $l$ which is contained in $\mathbb{Q}\left(\zeta_{l^{2}}\right)$. No other prime execpt $l$ is ramified in $L_{0}$. If $L$ is a Galois extension of $\mathbb{Q}$ with $\mathcal{G}(L / \mathbb{Q}) \cong C_{l}^{r}$ which does not contain $L_{0}$, then $\mathcal{G}\left(L L_{0} / \mathbb{Q}\right) \cong C_{l}^{r+1}$. Hence, by the preceding paragraph $\left|\operatorname{Ram}\left(L L_{0} / \mathbb{Q}\right) \backslash \mathbb{P}_{l}\right| \geq r$. Since $\operatorname{Ram}(L / \mathbb{Q})$ and $\operatorname{Ram}\left(L_{0} / \mathbb{Q}\right)=\{l\}$ are disjoint, we conclude that $\operatorname{Ram}(L / \mathbb{Q})$ contains at least $r$ elements.

Similarly, let $L$ be a Galois extension of $\mathbb{F}_{q}(t)$ with Galois group isomorphic to $C_{l}^{r}$ which is regular over $\mathbb{F}_{q}$. Then $L$ is disjoint from the unique unramified extension $\mathbb{F}_{q^{l}}$ of degree $l$ of $\mathbb{F}_{q}(t)$. As in the preceding paragraph, $\left|\operatorname{Ram}\left(L / \mathbb{F}_{q}(t)\right)\right| \geq r$.

Thus, if $K=\mathbb{Q}$ or $K=\mathbb{F}_{q}(t)$, Theorem 7.4 gives the most economic realization of $C_{l}^{r}$ in terms of number of ramified primes.

\section{$\S 8$. Estimates}

In this section we consider the case where $K$ is a function field of one variable over the field $K_{0}=\mathbb{F}_{q}$, where $q$ is a prime power. Our goal is to estimate some of the invariants of the field $L$ of Theorem 7.4.

Data 8.1. We fix the following notation for the whole section:

$$
\begin{aligned}
t= & \text { transcendental element } \\
& \quad \text { over } K \text { which we also consider as a variable } \\
K= & \text { finite separable extension of } K_{0}(t) \text { which is regular over } K_{0} \\
g_{K}= & \text { the genus of } K \\
d= & {\left[K: K_{0}(t)\right] }
\end{aligned}
$$




$$
\begin{aligned}
l & =\text { prime element } \neq \operatorname{char}(K) \\
n & =\text { positive integer } \\
d_{n} & =\left[K_{0}\left(\zeta_{l^{n}}\right): K_{0}\right] \\
S_{0} & =\text { basic set for } K(\text { Data } 1.1) ; \text { put } s_{0}=\left|S_{0}\right| ; \\
N^{*} & =\text { basic extension of } K(\text { Definition } 5.4) \\
S_{1} & =\text { exceptional set of primes for } K .
\end{aligned}
$$

Note that $s_{1}=\left|S_{1}\right|=\operatorname{rank}_{l} K($ Data 5.6$)$.

Lemma 8.2. Let $N$ be a finite Galois extension of $K$. Denote the algebraic closure of $K_{0}$ in $N$ by $K_{0}^{\prime}$. Let $\mathcal{C}$ be a conjugacy class in $\mathcal{G}(N / K)$, and let $c=|\mathcal{C}|$. Let $k$ be a multiple of $\left[K_{0}^{\prime}: K_{0}\right]$. Denote the number of primes $\mathfrak{q}$ of $K$ which do not ramify over $K_{0}(t)$ nor in $N$, are of degree $k$, and such that $\left(\frac{N / K}{q}\right)=\mathcal{C}$ by $\nu$. Let $\nu_{0}$ be a positive integer and suppose that

$$
\begin{aligned}
& k \log q \geq \max \left\{2 \log \left(2 g_{N}+(d+1)\left[N: K^{\prime}\right]+3\right)\right. \\
&\left.4 \log \left(3 g_{K}+1\right), 2 \log \left(\frac{\nu_{0} k}{c}\left[N: K^{\prime}\right]\right)\right\}
\end{aligned}
$$

then $\nu>\nu_{0}$.

Proof. Let $K^{\prime}=K K_{0}^{\prime}$ and let $m=\left[N: K^{\prime}\right]$. By (8.1), $q^{k / 2} \geq \nu_{0} k m / c$ and $q^{k / 2} \geq 2 g_{N}+2 m+3$. Also, (13.1) holds with $K$ and $N$, respectively instead of $E$ and $F$. Hence, by Corollary 13.5

$$
\begin{aligned}
\nu & >\frac{c}{k m} \cdot q^{k}-\frac{2 c}{k m} \cdot\left(m+g_{N}+1\right) \cdot q^{k / 2} \\
& =\frac{c}{k m} q^{k / 2} \cdot\left(q^{k / 2}-2\left(g_{N}+m+1\right)\right) \\
& \geq \nu_{0} \cdot 1=\nu_{0} .
\end{aligned}
$$

This proves our claim.

For a finite set $T$ of primes of a function field $K / K_{0}$ we write

$$
\operatorname{deg}(T)=\sum_{\mathfrak{p} \in T} \operatorname{deg}(\mathfrak{p})
$$


LEMMA 8.3. Let $L / K$ be a finite tamely ramified extension of function fields of one variable over the same constant field. Then their genera satisfy the following inequality:

$$
\frac{2 g_{L}-2}{[L: K]} \leq\left(2 g_{K}-2\right)+\operatorname{deg}(\operatorname{Ram}(L / K))
$$

Proof. The Riemann-Hurwitz genus formula for $L / K$ is

$$
2 g_{L}-2=[L: K]\left(2 g_{K}-2\right)+\sum_{\mathfrak{p} \in \operatorname{Ram}(L / K)} \sum_{\mathfrak{P} \mid \mathfrak{p}}(e(\mathfrak{P} / \mathfrak{p})-1) \operatorname{deg}(\mathfrak{P})
$$

[FrJ, p. 24]. In the second sum $\mathfrak{P}$ ranges over all prime divisors of $L$ which lie over $\mathfrak{p}$ and $e(\mathfrak{P} / \mathfrak{p})$ denotes the relative ramification index. We also denote the relative residue degree of $\mathfrak{P} / \mathfrak{p}$ by $f(\mathfrak{P} / \mathfrak{p})$. Then

$$
\begin{aligned}
\sum_{\mathfrak{p} \in \operatorname{Ram}(L / K)} \sum_{\mathfrak{P} \mid \mathfrak{p}}(e(\mathfrak{P} / \mathfrak{p})-1) \operatorname{deg}(\mathfrak{P}) \\
\leq \sum_{\mathfrak{p} \in \operatorname{Ram}(L / K)}\left(\sum_{\mathfrak{P} / \mathfrak{p}} e(\mathfrak{P} / \mathfrak{p}) f(\mathfrak{P} / \mathfrak{p})\right) \operatorname{deg}(\mathfrak{p}) \\
=\sum_{\mathfrak{p} \in \operatorname{Ram}(L / K)}[L: K] \operatorname{deg}(\mathfrak{p}) \\
=[L: K] \operatorname{deg}(\operatorname{Ram}(L / K))
\end{aligned}
$$

Hence, inequality (8.3) follows from (8.4).

LEMMA 8.4. Let $T$ be a finite set of primes of $K$. Suppose that $\zeta_{l} \notin$ $K$. Let $L / K$ be a finite l-extension such that $L$ is regular over $K_{0}$ and let $S=\operatorname{Ram}(L / K) \cup T$. Consider elements $a_{1}, \ldots, a_{r}$ of $K_{S}$ which are multiplicatively independent modulo $K_{S}^{l}$ and let $N=L\left(\zeta_{l^{n}}, \sqrt[l]{a_{1}}, \ldots, \sqrt[l]{a_{r}}\right)$. Then

$$
\frac{2 g_{N}-1}{l^{r}[L: K]} \leq 2 g_{K}-2+\operatorname{deg}(S)
$$

Proof. We apply Lemma 8.3 to $N / K\left(\zeta_{l^{n}}\right)$ instead of to $L / K$.

Let $K_{0}^{\prime}=K_{0}\left(\zeta_{l^{n}}\right), K^{\prime}=K\left(\zeta_{l^{n}}\right)$, and $L^{\prime}=L\left(\zeta_{l^{n}}\right)$. Then $K^{\prime} / K_{0}^{\prime}$ is a regular extension which is obtained from the function field $K / K_{0}$ by a finite separable extension of the field of constants. Hence $g_{K^{\prime}}=g_{K}$ [Deu, p. 132]. Let $S^{\prime}$ be the set of primes of $K^{\prime} / K_{0}^{\prime}$ which lie over $S$. Since each prime of $K^{\prime}$ is unramified over $K_{0}^{\prime}$, we have $\operatorname{deg}\left(S^{\prime}\right)=\operatorname{deg}(S)$ [Deu, 132]. 
By Remark 5.3, $N$ is a regular extension of $K_{0}^{\prime}$ and by Lemma 5.2, $\left[N: K^{\prime}\right]=l^{r}[L: K]$. Since $l$ is relatively prime to $\operatorname{char}(K)$, the extension $N / K^{\prime}$ is tamely ramified. If a prime $\mathfrak{p}$ of $K^{\prime} / K_{0}^{\prime}$ does not belong to $S$ and $\mathfrak{P}$ is an extension of $\mathfrak{p}$ to $L^{\prime}$, then $v_{\mathfrak{P}}\left(a_{i}\right)=v_{\mathfrak{p}}\left(a_{i}\right)=0$. Hence, $\mathfrak{P}$ is unramified in $L^{\prime}\left(\sqrt[l]{a_{i}}\right)$ (a consequence of [CaF, p. 32, Prop. 1]) and therefore $\mathfrak{p}$ is unramified in $N$. Thus, $\operatorname{Ram}\left(N / K^{\prime}\right) \subseteq S^{\prime}$.

It follows that (8.5) is a consequence of (8.3).

Lemma 8.5. Let $T$ be a finite set of primes of $K$. Suppose that $\zeta_{l} \notin K$. Let $L / K$ be a Galois extension of degree $l^{m}$ such that $L$ is regular over $K_{0}$ and let $S=\operatorname{Ram}(L / K) \cup T$. Consider elements $a_{1}, \ldots, a_{s}$ of $K_{S}$ which are multiplicatively independent modulo $K_{S}^{l}$ and let $N=$ $L\left(\zeta_{l^{n}}, \sqrt[l]{a_{1}}, \ldots, \sqrt[l]{a_{s}}\right)$. Suppose that $k$ is a multiple of $d_{n}$ such that $\operatorname{deg}(\mathfrak{p}) \leq k$ for each $\mathfrak{p} \in S$. Let

$$
\mu=3 g_{K}+d+1+|S|
$$

and let $\mathcal{C}$ be a conjugacy class in $\mathcal{G}\left(N / K\left(\zeta_{l^{n}}\right)\right)$. Suppose that

$$
k \log q \geq 4 \log 8+4(m+s) \log l+4 \log \mu .
$$

Then there exists $\mathfrak{q} \in \mathbb{P} \backslash S$ such that $\operatorname{deg}(\mathfrak{q})=k$ and $\left(\frac{N / K}{\mathfrak{q}}\right)=\mathcal{C}$.

Proof. Let $K_{0}^{\prime}=K_{0}\left(\zeta_{l^{n}}\right)$ and $K^{\prime}=K\left(\zeta_{l^{n}}\right)$. By Lemma $5.2,\left[N: K^{\prime}\right]=$ $l^{m+s}$. If $m+s=0$, then $N=K\left(\zeta_{l^{n}}\right)$. Then $g_{N}=g_{K}$ and the constant $m$ of Lemma 8.2 becomes 1 . Let $\nu$ be the number of $\mathfrak{q} \in \mathbb{P}$ such that $\operatorname{deg}(\mathfrak{q})=k$ and $\left(\frac{N / K}{q}\right)=\mathcal{C}$. We have to prove that $\nu>|S|$. Indeed, the inequality of Lemma 8.2 simplifies to $\nu>\frac{q^{k / 2}}{k}\left(q^{k / 2}-2 g_{K}-2\right)$. Since $q^{k / 2} \geq 2^{k / 2} \geq k$ and $q^{k / 2} \geq \mu^{2}$ (by (8.7)), we have $\nu>\mu^{2}-\nu>|S|$.

So, assume from now on that $m+s \geq 1$. By Lemma 8.2, it suffices to prove (8.1) with $\nu_{0}=|S|$.

The inequality $k \log q \geq 4 \log \left(2 g_{K}+1\right)$ follows from (8.6) and (8.7). Hence, it suffices to prove

$$
\begin{aligned}
& \frac{k}{2} \log q \geq \log \left(2 g_{N}+(d+1) l^{m+s}+3\right) \\
& \frac{k}{2} \log q \geq \log \left(k l^{m+s}|S|\right) .
\end{aligned}
$$


By Lemma 8.4 and by the assumption ' $\operatorname{deg}(\mathfrak{p}) \leq k$ for each $\mathfrak{p} \in S$ ' we have

$$
2 g_{N}-2 \leq l^{m+s}\left(2 g_{K}-2+\operatorname{deg}(S)\right) \leq l^{m+s}\left(2 g_{K}-2+k|S|\right) .
$$

Since $5 \leq 2 l^{m+s},(8.10)$ gives

$$
2 g_{N}+(d+1) l^{m+s}+3 \leq l^{m+s}\left(2 g_{K}+k|S|+d+1\right) .
$$

Nest observe that $\frac{\log x}{x}$ is a decreasing function for $x \geq e$ and that $\frac{\log 16}{16}=\frac{\log 2}{4}$. Hence, for $k \geq 16, \log k \leq \frac{k}{4} \log 2 \leq \frac{k}{4} \log q$. If $k \leq 16$, then by (8.6), $\mu \geq 2$ and then by (8.7), $k \log q \geq 4 \log (8 \mu) \geq 4 \log k$. Thus, in each case $\log k \leq \frac{k}{4} \log q$. It follows from (8.7) that

$$
\begin{aligned}
\log k+(m+s) \log l+\log \mu & \leq \frac{k}{4} \log q+(m+s) \log l+\log \mu \\
& \leq \frac{k}{4} \log q+\frac{k}{4} \log q=\frac{k}{2} \log q .
\end{aligned}
$$

Hence, by (8.11)

$$
\begin{aligned}
& \log \left(2 g_{N}+(d+1) l^{m+s}+3\right) \\
& \leq(m+s) \log l+\log k+\log \left(2 g_{K}+|S|+d+1\right) \\
&=\log k+(m+s) \log l+\log \mu \leq \frac{k}{2} \log q
\end{aligned}
$$

which proves (8.8). Finally, (8.9) follows from (8.13) and the inequality $|S| \leq \mu$.

THEOREM 8.6. Let $K$ be a function field of one variable over $\mathbb{F}_{q}$ and let $S_{0}$ and $S_{1}$ be sets of primes as in Data 8.1. Let $G$ be a group of order $l^{m}$ and let $n \geq m$. Suppose that $\zeta_{l} \notin K$. Then $K$ has an $n$-Scholz extension $L$ which is regular over $\mathbb{F}_{q}$ such that $\mathcal{G}(L / K) \cong G,\left|\operatorname{Ram}(L / K) \cup S_{1}\right|=$ $m+\operatorname{rank}_{l} K$, and each $\mathfrak{p} \in S_{0}$ totally decomposes in $L$.

Moreover, let $\mu=3 g_{K}+d+m+s_{0}+\operatorname{rank}_{l} K+1$ and let $k$ be a multiple of $d_{n}$ such that

$$
k \log q \geq 4 \log 8+4\left(m+\operatorname{rank}_{l} K\right) \log l+4 \log \mu .
$$

Then we can choose $S_{1}$ and $L$ such that $\operatorname{deg}(\mathfrak{p})=k$ for each $\mathfrak{p} \in \operatorname{Ram}(L / K) \cup$ $S_{1}$. The genus of $L$ is estimated by

$$
2 g_{L}-2 \leq l^{m}\left(2 g_{K}-2+\left(m+\operatorname{rank}_{l}(K)\right) k\right) .
$$


Proof. Denote $\mathbb{F}_{q}$ by $K_{0}$. Assume without loss that $m \geq 1$. Embed $C_{l}$ in the center of $G$ and let $\bar{G}=G / C_{l}$. By induction, $K$ has an $n$-Scholz extension $L$ which is regular over $K_{0}$ such that $\mathcal{G}(L / K) \cong \bar{G}$, and the above conditions are satisfied for $m-1$ instead of for $m$. Now consider the following central embedding problem

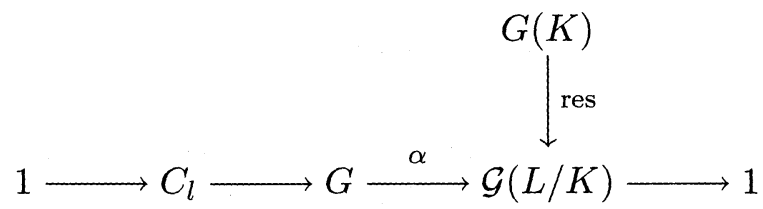

Lemmas 4.2 and 4.4 give a solution $\psi$ to $(8.15)$, which however, need not be $n$-Scholz. Use Lemma 6.2 to replace $\psi$ by another solution, if necessary, to assume that $\operatorname{Ram}(\psi) \subseteq \operatorname{Ram}(L / K) \cup S_{1}$. Then Lemma 7.2 gives a prime $\mathfrak{q} \notin S_{0} \cup S_{1} \cup \operatorname{Ram}(L / K)$ and an $n$-Scholz solution $\psi$ to $(8.15)$ such that $\operatorname{Ram}(\varphi)=\operatorname{Ram}(\psi) \cup\{\mathfrak{q}\}$. The fixed field $L^{*}$ of $\operatorname{Ker}(\varphi)$ is an $n$-Scholz extension of $K$ with $\mathcal{G}\left(L^{*} / L\right) \cong G$ and $\operatorname{Ram}\left(L^{*} / K\right)=\operatorname{Ram}(\psi) \cup\{\mathfrak{q}\}$. In particular $L^{*} / L$ ramifies. Hence, as $L$ is regular over $K_{0}$, so is $L^{*}$.

Now suppose that $(8.13)$ holds. Then apply Lemma 8.5 to choose the exceptional set $S_{1}$ with primes of degree $k$. Here we follow the construction of Data 5.6 with $r=\operatorname{rank}_{l}(K)$ and $s=\operatorname{rank}_{\infty}(K)=0$. Let $N^{*}$ and $w_{i}$ be as in Lemma 5.5. Then we choose a generator $\tau_{i}$ for $\mathcal{G}\left(N^{*} / N_{i}\right)$ and apply Lemma 8.5 to choose $\mathfrak{p}_{i}^{*} \in \mathbb{P} \backslash \operatorname{Ram}\left(N^{*} / K\right) \cup S_{0}$ such that $\left(\frac{N^{*} / K}{\mathfrak{p}_{i}^{*}}\right)=\operatorname{Con}\left(\sigma_{i}\right)$, $i=1, \ldots, r$.

In order to choose $\mathfrak{q} \notin S_{0} \cup S_{1} \cup \operatorname{Ram}(L / K)$ as in Lemma 7.2 we have to apply Lemma 7.1. The latter Lemma chooses generators $a_{1}, \ldots, a_{s}$ for $K_{S}$ modulo $K_{S}^{l}$, puts $N=L\left(\zeta_{l^{n}}, \sqrt[l]{a_{1}}, \ldots, \sqrt[l]{a_{s}}\right)$ and chooses $\sigma \in \mathcal{G}\left(N / L\left(\zeta_{l^{n}}\right)\right)$ in a special way (Part D, after the proof). Then it applies the Chebotarev density theorem to choose $\mathfrak{q} \in \mathbb{P} \backslash S$ such that either $\left(\frac{N / K}{\mathfrak{q}}\right)=1$ (in Part B) or $\left(\frac{N / K}{q}\right)=\operatorname{Con}(\sigma)$ (in Part D). Since (8.13) holds, we may apply Lemma 8.5, which is an effective version of the Chebotarev density theorem, and choose $\mathfrak{q}$ such that in addition to the above, $\operatorname{deg}(\mathfrak{q})=k$.

Finally, (8.14) follows from Lemma 8.3.

\section{$\S$ 9. Field of rational function}

The case where $K=\mathbb{F}_{q}(t)$ is simpler than the general one. In this case, in the notation of Data 8.1, we have 
(9.1) $d=1, h_{K}=1, N^{*}=\mathbb{F}_{q}\left(t, \zeta_{l^{n}}\right), \operatorname{Ram}\left(N^{*} / K\right)=\emptyset, \operatorname{rank}_{l}(K)=0$, $S_{1}=\emptyset$, and $g_{K}=0$. We take $S_{0}$ as a finite set of primes of $K / \mathbb{F}_{q}$ which contains $(t)_{\infty}$.

Note that $\zeta_{l} \notin K$ is equivalent to $l \nmid q-1$ and $l \neq \operatorname{char}(K)$ is equivalent to $l \nmid q$. Thus Theorem 8.6 specializes in this case to the following result:

THEOREM 9.1. Let $q$ be a prime power and let $l$ be a prime such that $l \nmid(q-1) q$. Let $G$ be a group of order $l^{n}$ and let $S_{0}$ be a finite set of primes of $\mathbb{F}_{q}(t)$ which contains $(t)_{\infty}$. Then $\mathbb{F}_{q}(t)$ has an $n$-Scholz extension $L$ which is regular over $\mathbb{F}_{q}$ such that $\mathcal{G}\left(L / \mathbb{F}_{q}(t)\right) \cong G,\left|\operatorname{Ram}\left(L / \mathbb{F}_{q}(t)\right)\right|=n$, and such that each $\mathfrak{p} \in S_{0}$ totally decomposes in $L$. Moreover, let $k$ be a multiple of $d_{n}$ such that $k \log q \geq 4 \log \left(8 l^{n}\left(1+n+\left|S_{0}\right|\right)\right)$. Then we can choose $L$ such that $\operatorname{deg}(\mathfrak{p})=k$ for each $\mathfrak{p} \in \operatorname{Ram}(L / K)$ and $2 g_{L}-2 \leq l^{n}(n k-2)$.

By the primitive element theorem, there exists a Galois polynomial $f \in \mathbb{F}_{q}[t, X]$ such that $\mathcal{G}\left(f(t, X), \mathbb{F}_{q}(t)\right) \cong G$. The degree of $f$ in $X$ is of course $l^{n}$. The following result will enable us to choose $f$ with bounded degree in $t$.

LEMMA 9.2. Let $K_{0}$ be an arbitrary field and consider a Galois extension $L$ of $K=K_{0}(t)$ of degree $d$ which is regular over $K_{0}$.

(a) Suppose that $(t)_{\infty}$ totally decomposes in L. Let $\mathfrak{p}$ be a prime divisor of $L / K_{0}$ which divides $(t)_{\infty}$ and let $x$ be an element of $L$ such that $(x)_{\infty}=k \mathfrak{p}$ for some positive integer $k$. Then $x$ is integral over $K_{0}[t]$, $L=K(x)$ and $f=\operatorname{irr}(x, K(t))$ has the form

$$
f(t, X)=X^{d}+a_{1}(t) X^{d-1}+\cdots+a_{d}(t) .
$$

with $a_{i} \in K_{0}[t]$ and $\operatorname{deg}\left(a_{i}(t)\right) \leq \operatorname{deg}\left(a_{1}(t)\right)=k, i=1, \ldots, d$.

(b) Conversely, suppose that $x \in L$ and that $f=\operatorname{irr}(x, K(t))$ is given by (9.1) such that $a_{i}(t) \in K_{0}[t], \operatorname{deg}\left(a_{1}(t)\right)>0$, and $\operatorname{deg}\left(a_{i}(t)\right) \leq$ $\operatorname{deg}\left(a_{1}(t)\right), i=1, \ldots, d$. Then $(t)_{\infty}$ totally decomposes in $L$.

Proof of (a). Denote the normalized valuation of $L / K_{0}$ that corresponds to $\mathfrak{p}$ by $v$. Then $v(x)=-k$ and $w(x) \geq 0$ for each other valuation $w$ of $L / K_{0}$. In particular, since $v^{\sigma} \neq v$, we have $v\left(x^{\sigma^{-1}}\right)=v^{\sigma}(x) \geq 0$ for each $\sigma \in \mathcal{G}(L / K), \sigma \neq 1$. Hence $x^{\sigma^{-1}} \neq x$ for each $\sigma \neq 1$, and therefore $L=K(t, x)$. 
In addition $w(x) \geq 0$ if $w(t) \geq 0$. Hence $x$ is integral over $K_{0}[t]$. In particular $f(t, X)=\operatorname{irr}(x, K(t)) \in K_{0}[t, X]$ is a monic polynomial in $X$. Since $L / K$ is a Galois extension, $f(t, X)$ decomposes into distinct linear factors over $L$ :

$$
f(t, X)=\prod_{\sigma \in G}\left(X-x^{\sigma}\right) .
$$

A comparison of (9.1) and (9.2) gives:

$$
a_{i}(t)=(-1)^{i} \sum_{S \in \mathcal{P}_{i}} \prod_{\sigma \in S} x^{\sigma}
$$

where $\mathcal{P}_{i}$ is the collection of all subsets of $G$ of cardinality $i$. Note that $v$ is unramified over $K$. Hence the restriction of $v$ to $K$ coincides with the valuation $v_{\infty}$ that corresponds to $(t)_{\infty}$. Since $\sigma=1$ appears at most once in each of the summands $\prod_{\sigma \in S} x^{\sigma}$, and since $v\left(x^{\sigma}\right)=0$ for $\sigma \neq 1$, this gives

$$
-\operatorname{deg}\left(a_{i}(t)\right)=v_{\infty}\left(a_{i}(t)\right)=v\left(a_{i}(t)\right) \geq \min _{S \in \mathcal{P}_{i}} \sum_{\sigma \in S} v\left(x^{\sigma}\right)=-k .
$$

Also, $-\operatorname{deg}\left(a_{1}(t)\right)=v_{\infty}\left(a_{1}(t)\right)=v\left(-x-\sum_{\sigma \neq 1} x^{\sigma}\right)=v(x)=-k$, as desired.

Proof of (b). Let $k=\operatorname{deg}\left(a_{1}(t)\right)$. Then $z=x / t^{k}$ satisfies

$$
z^{d}+b_{1}(t) z^{d-1}+b_{2}(t) z^{d-2}+\cdots+b_{d}(t)=0,
$$

where $b_{i}(t)=a_{i}(t) / t^{i k}$. As in (a), choose an extension $v$ of $v_{\infty}$ to a valuation of $L$, let $e=e\left(v / v_{\infty}\right)$ and let $\mathfrak{p}$ be a prime divsior of $L / K_{0}$ that corresponds to $v$. Then $v\left(b_{1}(t)\right)=0$ and $v\left(b_{i}(t)\right)=e\left(i k-\operatorname{deg}\left(a_{i}(t)\right)\right)>0$ for $i=2, \ldots, d$. Hence reduction of (9.4) modulo $\mathfrak{p}$ gives $\bar{z}^{d-1}(\bar{z}+b)=0$ for some $0 \neq b \in K_{0}$. By Hensel's Lemma, $h(Z)=Z^{d}+b_{1}(t) Z^{d-1}+\cdots+b_{n}(t)$ has a root in the completion $K_{0}\left(\left(t^{-1}\right)\right)$ of $K$ with respect to $(t)_{\infty}$. Since $L=K(z)$ is Galois over $K$, all roots of $h(Z)$ are in $K_{0}\left(\left(t^{-1}\right)\right)$. Conclude that $(t)_{\infty}$ totally decomposes in $L$.

LEMMA 9.3. Let $K_{0}$ be an arbitrary field and consider a Galois extension $L$ of $K=K_{0}(t)$ of degree $d$ which is regular over $K_{0}$. Suppose that $(t)_{\infty}$ totally decomposes in $L$. Then there exists $x \in L$ which is integral over $K_{0}[t]$ such that $L=K(x)$ and $f=\operatorname{irr}(x, K(t))=X^{d}+a_{1}(t) X^{d-1}+\cdots+a_{d}(t)$ with $a_{i} \in K_{0}[t]$ such that $0<\operatorname{deg}\left(a_{1}(t)\right) \leq g_{L}+1$ and $\operatorname{deg}\left(a_{i}(t)\right) \leq \operatorname{deg}\left(a_{1}(t)\right)$, $i=1, \ldots, d$. 
Proof. Let $G=\mathcal{G}(L / K)$. By assumption $(t)_{\infty}=\sum_{\sigma \in G} \mathfrak{p}^{\sigma}$ for some prime divisor $\mathfrak{p}$ of $L$ and $\mathfrak{p}^{\sigma} \neq \mathfrak{p}^{\tau}$ if $\sigma \neq \tau$. In particular $\operatorname{deg}(\mathfrak{p})=1$. For each $k$ consider the vector space $\mathcal{L}(k \mathfrak{p})=\{x \in L \mid(x)+k \mathfrak{p} \geq 0\}$ over $K$. We have $\operatorname{dim} \mathcal{L}(0 \cdot \mathfrak{p})=1$ and $\operatorname{dim} \mathcal{L}\left(\left(2 g_{L}-1\right) \mathfrak{p}\right)=g$ [FrJ, p. 20], $\mathcal{L}((k-1) \mathfrak{p}) \subseteq \mathcal{L}(k \mathfrak{p})$ and $\operatorname{dim} \mathcal{L}(l \mathfrak{p})-\operatorname{dim} \mathcal{L}(k \mathfrak{p}) \leq l-k$ if $l \geq k$ [FrJ, Chap. 2, Exer. 12]. In particular, $\operatorname{dim} \mathcal{L}(k \mathfrak{p})=1$ implies $k \leq g$. Hence, the first $k$ for which $\operatorname{dim} \mathcal{L}(k \mathfrak{p})=2$ satisfies $k \leq g+1$. For this $k$ there exists $x \in L$ such that $(x)_{\infty}=k \mathfrak{p}$. By Lemma 9.2, $x$ satisfies the requirements of the present lemma.

\section{$\S 10$. Bounds}

The goal of this section is to realize a given $l$-group $G$ over $\mathbb{F}_{q}(t)$ with $q$ large with a bound on all parameters involved in the realization. This will enable us to use model theory and to realize $G$ over $K_{0}(t)$ for any pseudo finite field $K_{0}$. In order to do this, we need to speak about the set of ramified primes in the first order language of fields. So, we have to express discriminants of field extensions in an elementary way.

Let $R$ be a Dedekind domain with a quotient field $K$, let $L$ be a finite Galois extension of $K$ with Galois group $G=\left\{\sigma_{1}, \ldots, \sigma_{d}\right\}$, and let $S$ be the integral closure of $R$ in $L$. The discriminant of $S / R$ is the ideal $\operatorname{Disc}(S / R)$ of $R$ which is generated by all determinants $\operatorname{Det}\left(\sigma_{i} w_{j}\right)^{2}$ where $w_{1}, \ldots, w_{d} \in S$ are linearly independent over $K$. If $\mathfrak{p}$ is a prime ideal of $R$, we can compute the $\mathfrak{p}$-component of the discriminant by localizing at p. That is $\operatorname{Disc}(S / R) R_{\mathfrak{p}}=\operatorname{Disc}\left(S_{\mathfrak{p}} / R_{\mathfrak{p}}\right)$ [La2, p. 65]. The set of prime ideals of $R$ which ramify in $L$ coincides with the set of prime divisors of $\operatorname{Disc}(S / R)$ [CaF, p. 22]. In particular, if $R=K_{0}[t]$ for some field $K_{0}$, and $(t)_{\infty}$ is unramified in $L$, then $\operatorname{Ram}(L / K)$ consists of the prime divisors of $\operatorname{Disc}(S / R)$.

The discriminant of a monic polynomial $f \in R[X]$ is given in terms of its roots $x_{1}, \ldots, x_{n}$ by the formula $\operatorname{Disc}(f)=(-1)^{d(d-1) / 2} \prod_{i \neq j}\left(x_{i}-x_{j}\right)$. It is an element of $R$ and equals $(-1)^{d(d-1) / 2} N_{L / K} f^{\prime}\left(x_{1}\right)$. One can compute $\operatorname{Disc}(f)$ in terms of the resultant of $f$ and its derivative by the formula $\operatorname{Resultant}\left(f, f^{\prime}\right)=(-1)^{d(d-1) / 2} \operatorname{Disc}(f)\left[\operatorname{La} 4\right.$, p. 211]. Resultant $\left(f, f^{\prime}\right)$ is a $(2 d-1) \times(2 d-1)$ determinant whose entries are the coefficients of $f$ and $f^{\prime}$. The only nonzero entries in the first column of this determinant are the leading coefficients of $f$ and $f^{\prime}$. In the case where $R=K_{0}[t]$, this leads to an estimate on the degree of $\operatorname{Disc}(f)$ : 
(10.1) Suppose that $R=K_{0}[t]$ and $\operatorname{char}\left(K_{0}\right) \nmid d$. If $f \in K_{0}[t, X]$ is a monic polynomial of degree $d$ in $X$ and $\operatorname{deg}_{t}(f) \leq m$, then $\operatorname{deg}(\operatorname{Disc}(f)) \leq$ $m^{2 d-2}$.

If $x$ is integral over $K, L=K(x)$, and $f=\operatorname{irr}(x, K)$, then $\operatorname{Disc}(f)$ is a multiple of $\operatorname{Disc}(S / R)$. If in addition $S=R[x]$, then $\operatorname{Disc}(S / R)=\operatorname{Disc}(f) R$ $[\mathrm{CaF}$, p. 17]. This situation occurs often in the local case as Lemma 10.1 reveals. Together with the local nature of the discriminant, this gives us a tool to handle disciminants.

LEMMA 10.1. Let $R$ be a discrete valuation ring of a field $K$ with a maximal ideal $\mathfrak{p}$ and a perfect residue field $\bar{K}$. Let $L$ be a finite Galois extension of $K$ with $|\bar{K}| \geq[L: K]$. Denote the integral closure of $R$ in $L$ by $S$. Then there exists $x \in S$, such that $S=R[x]$. Moreover, $S$ has only finitely many nonzero prime ideals $\mathfrak{q}_{1}, \ldots, \mathfrak{q}_{r}$. If $x^{\prime} \in S$ satisfies $x^{\prime} \equiv x \bmod \mathfrak{q}_{i}^{2}, i=1, \ldots, r$, then $S=R\left[x^{\prime}\right]$.

Proof. Denote the valuation of $K$ that corresponds to $R$ by $v$. The ring $S$ is a finitely generated $R$-module [La2, p. 6] and has only finitely many prime ideals $\mathfrak{q}_{1}, \ldots, \mathfrak{q}_{r}$. They correspond to the extensions $v_{1}, \ldots, v_{r}$ of $v$ to $L$. In particular each $\mathfrak{q}_{i}$ is a principal ideal [La2, p. 15].

For each $i$ let $K_{i}$ be the decomposition field of $\mathfrak{q}_{i}$ over $K$ and let $L_{i}$ be its inertia field. Since $\bar{K}$ is perfect, the residue fields satisfy $\bar{K}_{i}=\bar{K}=R / \mathfrak{p}$, $\bar{L}_{i}=S / \mathfrak{q}_{i}$, and $\left[\bar{L}_{i}: \vec{K}\right]=\left[L_{i}: K_{i}\right]$ [Ser, p. 32]. For the same reason there exists $y_{i} \in S \cap L_{i}$ with $\bar{L}_{i}=\bar{K}\left[\bar{y}_{i}\right]$. Then $g_{i}=\operatorname{irr}\left(y_{i}, K_{i}\right)$ satisfies $\bar{g}_{i}=\operatorname{irr}\left(\bar{y}_{i}, \bar{K}\right)$ and in particular $\bar{g}_{i}{ }^{\prime}\left(\bar{y}_{i}\right) \neq 0$. Observe that $g_{i} \in\left(S \cap L_{i}\right)[X]$.

We may replace each $y_{i}$ by $y_{i}+a$ with $a \in R$. Since $\bar{y}_{i}$ has $\left[L_{i}: K_{i}\right]$ conjugates over $\bar{K}$ and since $\sum_{i=1}^{r}\left[L_{i}: K_{i}\right] \leq[L: K] \leq|\bar{K}|$, we may assume, without loss, that $\bar{y}_{1}, \ldots, \bar{y}_{r}$ are pairwise nonconjugate over $\bar{K}$. In other words, $\bar{g}_{1}, \ldots, \bar{g}_{r}$ are distinct.

Choose now $\pi_{i} \in S$ such that $v_{i}\left(\pi_{i}\right)=1$ and let $x_{i}=y_{i}+\pi_{i}$. Then $g_{i}\left(x_{i}\right)=g_{i}^{\prime}\left(y_{i}\right) \pi_{i}+c_{i}$ for some $c_{i} \in S$ with $v_{i}\left(c_{i}\right)>1$. Hence $v_{i}\left(g_{i}\left(x_{i}\right)\right)=1$. Since $R$ is $v_{i}$-dense in $S \cap L_{i}$, there exists $h_{i} \in R[X]$ such that $v_{i}\left(h_{i}-g_{i}\right)>1$. Then $v_{i}\left(h_{i}\left(x_{i}\right)\right)=1$.

Use the chinese remainder theorem to choose $x \in S$ such that $v_{i}(x-$ $\left.x_{i}\right)>1, i=1, \ldots, r$. Then $x$ modulo $\mathfrak{p}_{i}=\mathfrak{q}_{i} \cap R[x]$ generates $\bar{L}_{i}$. Also, with $\pi_{i}^{\prime}=h_{i}(x) \in R[x]$ we have $v_{i}\left(\pi_{i}^{\prime}\right)=1$. It follows that $\mathfrak{q}_{i}=\pi_{i}^{\prime} S=\mathfrak{p}_{i} S$ and hence $\mathfrak{p}_{1}, \ldots, \mathfrak{p}_{r}$ are distinct. Since $\mathfrak{q}_{1}, \ldots, \mathfrak{q}_{r}$ are the only nonzero prime 
ideals of $S$ and $\mathfrak{q}_{i} \cap R[x]=\mathfrak{p}_{i}, \mathfrak{q}_{i}$ is the only prime ideal of $S$ which lies over $\mathfrak{p}_{i}$. Also, as $S / R[x]$ is an integral extension, each nonzero prime ideal of $R[x]$ lies under some $\mathfrak{q}_{i}$. Thus $\mathfrak{p}_{1}, \ldots, \mathfrak{p}_{r}$ are all nonzero prime ideals of $R[x]$.

It suffices now to prove that $S=R[x]$. To this end consider the local rings $R[x]_{i}=R[x]_{\mathfrak{p}_{i}}$ and $S_{i}=S_{\mathfrak{q}_{i}}$. Then $S_{i}$ is the unique valuation ring of $L$ that contains $R[x]_{i}$. It follows that $S_{i}$ is the integral closure of $R[x]_{i}$ in $L$ [La3, p. 14]. Hence $S_{i}$ is a finitely generated $R[x]_{i}$-Modul. Also, $\pi_{i}^{\prime}$ generates the maximal ideal of $S_{i}$ and $\pi_{i}^{\prime} \in R[x]_{i}$. By construction, $S_{i} / \pi_{i}^{\prime} S_{i}=\bar{L}_{i}=$ $R[x]_{i} / \mathfrak{p}_{i} R[x]_{i}$. It follows that $S_{i}=R[x]_{i}+\pi_{i}^{\prime} S_{i}$. By Nakayama's Lemma, $S_{i}=R[x]_{i}$. Conclude that $S=\bigcap_{i=1}^{r} S_{i}=\bigcap_{i=1}^{r} R[x]_{i}=R[x]$.

LEMMA 10.2. (Strong approximation theorem) Let $L$ be a function field of one variable over a field $K_{0}$. Let $Q$ be a finite set of primes of $L / K_{0}$ and let $\mathfrak{q}_{\infty}$ be a prime of $L / K_{0}$ which does not belong to $Q$. Suppose that for each $\mathfrak{q} \in Q$ we are given an element $y_{\mathfrak{q}} \in L$ and a positive integer $m_{\mathfrak{q}}$. Suppose also that $m$ satisfies

$$
m \cdot \operatorname{deg}\left(\mathfrak{q}_{\infty}\right)>2 g_{L}-2+\sum_{\mathfrak{q} \in Q} m_{\mathfrak{q}} \operatorname{deg}(\mathfrak{q})
$$

Then there exists $y \in L$ such that $v_{\mathfrak{q}}\left(y-y_{\mathfrak{q}}\right) \geq m_{\mathfrak{q}}$ for each $\mathfrak{q} \in Q, v_{\mathfrak{q}_{\infty}}(y) \geq$ $-m$, and $v_{\mathfrak{p}}(y) \geq 0$ for each prime $\mathfrak{p} \notin S \cup\left\{\mathfrak{q}_{\infty}\right\}$.

Proof. Consider the divisor $\mathfrak{a}=m \mathfrak{q}_{\infty}-\sum_{\mathfrak{q} \in Q} m_{\mathfrak{q}} \mathfrak{q}$ of $L / K_{0}$. In the adele ring $\mathbb{A}$ of $L / K_{0}$ consider the vector space

$$
\Lambda(\mathfrak{a})=\left\{\alpha \in \mathbb{A} \mid v_{\mathfrak{p}}(\alpha)+v_{\mathfrak{p}}(\mathfrak{a}) \geq 0 \text { for every } \mathfrak{p}\right\} .
$$

By $(10.2), \operatorname{deg}(\mathfrak{a})>2 g_{L}-2$. Hence, by the Riemann-Roch theorem, $\operatorname{dim}(\mathbb{A} /(\Lambda(\mathfrak{a})+L))=0[\mathrm{FrJ}$, Sec. 2.6]. It follows that $\mathbb{A}=\Lambda(\mathfrak{a})+L$. Define $\eta \in \mathbb{A}$ by $\eta_{\mathfrak{q}}=y_{\mathfrak{q}}$ for $\mathfrak{q} \in Q$ and $\eta_{\mathfrak{p}}=0$ for $\mathfrak{p} \notin Q$. Then there exists $y \in L$ such that $y-\eta \in \Lambda(\mathfrak{a})$. This $y$ satisfies the requirements of the Lemma.

Notation 10.3. (The set $\mathcal{F}_{k}(L / K)$ ) Let $K_{0}$ be a field, let $K=K_{0}(t)$, let $L$ be a Galois extension of $K$ of degree $d$, and let $k$ be a positive integer. We define $\mathcal{F}_{k}\left(L / K_{0}\right)$ to be the set of all absolutely irreducible polynomials

$$
h(T, X)=X^{d}+c_{1}(T) X^{d-1}+\cdots+c_{d}(T)
$$


with $c_{i}(T) \in K_{0}[T]$ such that $0<\operatorname{deg}\left(c_{1}(T)\right) \leq(k+d)^{2 d}, \operatorname{deg}\left(c_{i}(T)\right) \leq$ $\operatorname{deg}\left(c_{1}(T)\right), i=1, \ldots, d$, and $L=K(z)$ with $h(t, z)=0$.

Lemma 10.4. Let $K_{0}$ be a perfect field, $R=K_{0}[t]$, and $K=K_{0}(t)$. Consider a Galois extension $L$ of $K$ of degree $d>1$ which is regular over $K_{0}$ and let $S$ be the integral closure of $R$ in $L$.

Suppose that $(t)_{\infty}$ totally decomposes in $L$, $\operatorname{char}\left(K_{0}\right) \nmid d$, and $\left|K_{0}\right| \geq d$. Suppose that $\mathcal{F}_{k}(L / K)$ contains a polynomial $f(T, X)=X^{d}+a_{1}(T) X^{d-1}+$ $\cdots+a_{d}(T)$ with $\operatorname{deg}\left(a_{1}(T)\right)=k>0$. Then the following two statements hold:

(a) There exists $g \in \mathcal{F}_{k}(L / K)$ such that

$$
\operatorname{Disc}(S / R)=\operatorname{gcd}(\operatorname{Disc}(f(t, X)), \operatorname{Disc}(g(t, X))) R .
$$

(b) Let $f_{1}, g_{1} \in \mathcal{F}_{k}(L / K)$ be polynomials such that

$$
d_{1}(t)=\operatorname{gcd}\left(\operatorname{Disc}\left(f_{1}(t, X)\right), \operatorname{Disc}\left(g_{1}(t, X)\right)\right)
$$

divides $\operatorname{Disc}(h(t, X))$ for each $h \in \mathcal{F}_{k}(L / K)$. Then $\operatorname{Disc}(S / R)=d_{1}(t) R$.

Proof of (a). The total degree of $f(T, X)$ is $k+d-1$. Hence, by [FrJ, Cor. 4.8]

$$
g_{L} \leq \frac{1}{2}(k+d-2)(k+d-3)<\frac{1}{2}(k+d)^{2} .
$$

Denote the set of prime divisors of $K / K_{0}$ which correspond to the irrreducible factors of $\operatorname{Disc}(f(t, X))$ by $P$ and note that $(t)_{\infty} \notin P$. By (10.1)

$$
\sum_{\mathfrak{p} \in P} \operatorname{deg}(\mathfrak{p}) \leq \operatorname{deg}(\operatorname{Disc}(f(t, X))) \leq k^{2 d-2} .
$$

For each $\mathfrak{p} \in P$ consider the localization $R_{\mathfrak{p}}$ and $S_{\mathfrak{p}}$ of $R$ and $S$, respectively, at $\mathfrak{p}$. Then $R_{\mathfrak{p}}$ is a discrete valuation ring with residue field which contains $K_{0}$, and therefore of cardinality at least $d$, and $S_{\mathfrak{p}}$ is its integral closure in $L$. By Lemma 10.1 there exists $y_{\mathfrak{p}} \in S_{\mathfrak{p}}$ such that $S_{\mathfrak{p}}=R_{\mathfrak{p}}\left[y_{\mathfrak{p}}\right]$. Moreover, let $Q_{\mathfrak{p}}$ be the set of prime divisors of $\mathfrak{p}$ in $L$. Then, $S_{\mathfrak{p}}=R_{\mathfrak{p}}[y]$ for each $y \in S_{\mathfrak{p}}$ which satisfies $v_{\mathfrak{q}}\left(y-y_{\mathfrak{p}}\right)>1$ for each $\mathfrak{q} \in Q_{\mathfrak{p}}$. Since $\mathfrak{p}=\sum_{\mathfrak{q} \in Q_{\mathfrak{p}}} e(\mathfrak{q} / \mathfrak{p}) \mathfrak{q}$, we have

$$
\sum_{\mathfrak{q} \in Q_{\mathfrak{p}}} \operatorname{deg}(\mathfrak{q}) \leq \sum_{\mathfrak{q} \in Q_{\mathfrak{p}}} e(\mathfrak{q} / \mathfrak{p}) \operatorname{deg}(\mathfrak{q})=\operatorname{deg}(\mathfrak{p})
$$


Choose now a prime divsor $\mathfrak{q}_{\infty}$ of $L$ which divides $(t)_{\infty}$ and let $m=$ $2 g_{L}-1+\sum_{\mathfrak{p} \in P} \sum_{\mathfrak{q} \in Q_{\mathfrak{p}}} 2 \operatorname{deg}(\mathfrak{q})$. By (10.4), (10.5), and (10.6)

$$
m \leq(k+d)^{2}+2 k^{2 d-2} \leq 3(k+d)^{2 d-2} \leq(k+d)^{2 d}
$$

By assumption, $\operatorname{deg}\left(\mathfrak{q}_{\infty}\right)=1$. Hence, by Lemma 10.2 with $Q=\bigcup_{\mathfrak{p} \in P} Q_{\mathfrak{p}}$ and $m_{\mathfrak{q}}=2$, there exists $y \in L$ such that $v_{\mathfrak{q}}\left(y-y_{\mathfrak{p}}\right) \geq 2$ for each $\mathfrak{p} \in P$ and each $\mathfrak{q} \in Q_{\mathfrak{p}}, v_{\mathfrak{q}_{\infty}}(y) \geq-m$, and $v_{\mathfrak{q}}(y) \geq 0$ for each $\mathfrak{q} \notin Q \cup\left\{\mathfrak{q}_{\infty}\right\}$. Since $v_{\mathfrak{q}}\left(y_{\mathfrak{p}}\right) \geq 0$ for each $\mathfrak{p} \in P$ and each $\mathfrak{q} \in Q_{\mathfrak{p}}$, we have $v_{\mathfrak{q}}(y) \geq 0$ for each prime of $\mathfrak{q} \neq \mathfrak{q}_{\infty}$. Hence $y \in S$. Also, $S_{\mathfrak{p}}=R_{\mathfrak{p}}[y]$ for each $\mathfrak{p} \in P$. In particular $L=K(y)$. Hence $y \notin K_{0}$ and therefore there exists a positive integer $k_{0} \leq m$ such that $(y)_{\infty}=k_{0} \mathfrak{q}_{\infty}$.

Let $g=\operatorname{irr}(y, K) \in R[X]$. Then $g(t, X)$ is monic and Galois in $X$, and since $L / K_{0}$ is regular, $g(T, X)$ is absolutely irreducible. By Lemma 10.2(a) applied to $y, g$, and $k_{0}$ instead of to $x, f$, and $k$ and by $(10.7), g(T, X)=$ $X^{d}+b_{1}(T) X^{d-1}+\cdots+b_{d}(T)$ with $b_{i}(T) \in K_{0}[T]$ and $\operatorname{deg}\left(b_{i}(T)\right) \leq \operatorname{deg}\left(b_{1}(T)\right)$ $=k_{0} \leq m \leq(k+d)^{2 d}$. Hence $g \in \mathcal{F}_{k}(L / K)$.

In order to conclude the proof of (a) recall first that the left hand side of (10.3) divides its right hand side. To prove the other direction consider a prime divisor $\mathfrak{p} \neq(t)_{\infty}$ of $K / K_{0}$. Then we may identify $\mathfrak{p}$ with a nonzero prime ideal of $R$. If $\mathfrak{p} \in P$, then $\operatorname{Disc}(S / R) R_{\mathfrak{p}}=\operatorname{Disc}\left(S_{\mathfrak{p}} / R_{\mathfrak{p}}\right)=$ $\operatorname{Disc}\left(R_{\mathfrak{p}}[y] / R_{\mathfrak{p}}\right)=\operatorname{Disc}(g(t, X)) R_{\mathfrak{p}}$. If $\mathfrak{p} \notin P$, then $v_{\mathfrak{p}}(\operatorname{Disc}(f(t, X)))=0$ and therefore $v_{\mathfrak{p}}(\operatorname{Disc}(S / R))=0$. Thus, in each case the value of $v_{\mathfrak{p}}$ at both sides of (10.3) is the same. So, (a) holds.

Proof of (b). Again, $\operatorname{Disc}(S / R)$ divides both $\operatorname{Disc}\left(f_{1}(t, X)\right)$ and $\operatorname{Disc}\left(g_{1}(t, X)\right)$ and therefore also $d_{1}(t)$. Conversely, by assumption, $d_{1}(t)$ divides both $\operatorname{Disc}(f(t, X))$ and $\operatorname{Disc}(g(t, X))$. Hence, by $(10.3), d_{1}(t)$ $\operatorname{Disc}(S / R)$. Conclude that $\operatorname{Disc}(S / R)=d_{1}(t) R$, as desired.

THEOREM 10.5. Let $q$ be a prime power, let $G$ be a group of order $l^{n}$ with $l$ a prime, and let $t$ be a transcendental element over $\mathbb{F}_{q}$. Suppose that $l \neq \operatorname{char}\left(\mathbb{F}_{q}\right), \zeta_{l} \notin \mathbb{F}_{q}$, and $q>l^{4 n+4}$. Then there exist absolutely irreducible polynomials $f, g \in \mathbb{F}_{q}[T, X]$ which are monic and Galois in $X$ such that

(a) $f(T, X)=X^{l^{n}}+a_{1}(T) X^{l^{n}-1}+\cdots+a_{l^{n}}(T), 0<\operatorname{deg}\left(a_{1}(T)\right) \leq \frac{1}{2} n l^{2 n}$, and $\operatorname{deg}\left(a_{i}(T)\right) \leq \operatorname{deg}\left(a_{1}(T)\right), i=1, \ldots, l^{n}$,

(b) $f(t, X)$ and $g(t, X)$ have the same splitting field $L$ over $K=\mathbb{F}_{q}(t) ; L$ is obtained by adjoining one root of $f(t, X)$ or of $g(t, X)$ to $K$, 
(c) $L$ is a regular extension of $\mathbb{F}_{q}$ and $\mathcal{G}(L / K) \cong G$,

(d) $g \in \mathcal{F}_{k}(L / K)$, where $k=\operatorname{deg}\left(a_{1}(T)\right)$,

(e) $(t)_{\infty}$ totally decomposes in $L$; in particular, $L$ has $l^{n}$ prime divisors of degree 1 ,

(f) $g_{L}<\frac{1}{2} n l^{2 n}$

(g) $|\operatorname{Ram}(L / K)|=n$ and $\operatorname{deg}(\mathfrak{p})=\left[\mathbb{F}_{q}\left(\zeta_{l^{n}}\right): \mathbb{F}_{q}\right]$ for each $\mathfrak{p} \in \operatorname{Ram}(L / K)$,

(h) Let $R=K_{0}[t]$ and let $S$ be the integral closure of $R$ in $L$. Then $\operatorname{Disc}(S / R)=\operatorname{gcd}(\operatorname{Disc}(f(t, X)), \operatorname{Disc}(g(t, X))) R$. Thus $\operatorname{Ram}(L / K)$ consists of those primes $\neq(t)_{\infty}$ of $K / K_{0}$ that divide both $\operatorname{Disc}(f(t, X))$ and $\operatorname{Disc}(g(t, X))$.

Proof. Assume without loss that $n \geq 1$. Since $\zeta_{l} \notin \mathbb{F}_{q}$, we have $l \geq 3$ and $2 \leq d_{n}=\left[\mathbb{F}_{q}\left(\zeta_{l^{n}}\right): \mathbb{F}_{q}\right] \leq l^{n}$. Then $q^{d_{n} / 4} \geq q^{1 / 2}>l^{2+2 n}>8 l^{n}(2+n)$. Hence, Theorem 9.1 with $k=d_{n}$ and $S_{0}=\left\{(t)_{\infty}\right\}$ gives a Galois extension $L$ of $K$ with $\mathcal{G}(L / K) \cong G$ which is regular over $\mathbb{F}_{q}$ such that $2 g_{L}-2 \leq$ $l^{n}\left(n d_{n}-2\right),|\operatorname{Ram}(L / K)|=n, \operatorname{deg}(\mathfrak{p})=d_{n}$ for each $\mathfrak{p} \in \operatorname{Ram}(L / K)$ and $(t)_{\infty}$ totally decomposes in $L$. Hence $g_{L}<\frac{1}{2} n l^{2 n}$ and so (f) is true. By Lemma 9.3, there exists $x \in L$ which is integral over $\mathbb{F}_{q}[t]$ such that $L=$ $\mathbb{F}_{q}(t, x)$ and $f(t, X)=\operatorname{irr}(x, K)$ satisfies (a). In particular $f(t, X)$ is monic and Galois in $X$. Since $L / \mathbb{F}_{q}$ is regular, $f(T, X)$ is absolutely irreducible.

Since $q>l^{4 n+4}>l^{n}=[L: K]$, Lemma 10.4, with $d=l^{n}$ gives an absolutely irreducible polynomial $g \in \mathcal{F}_{k}(L / K)$ such that $\operatorname{Disc}(S / R)$ is the greatest common divisor of the ideal of $\mathbb{F}_{q}[t]$ generated by $\operatorname{Disc}(f(t, X))$ and $\operatorname{Disc}(g(t, X))$. Since $(t)_{\infty}$ is unramified in $L$ this gives (h) and concludes the proof of the theorem.

\section{$\S 11$. Pseudo finite fields}

A field $K_{0}$ is pseudo finite it satisfies one of the following equivalent conditions $[\mathrm{Ax}$, Thm. 9]:

(11.1a) $K_{0}$ is a perfect, $G\left(K_{0}\right) \cong \hat{\mathbb{Z}}$, and each nonempty absolutely irreducible variety which is defined over $K_{0}$ has a $K_{0}$-rational point (Thus, in the terminology of [FrJ], $K_{0}$ is a perfect, 1-free PAC field.) 
(11.1b) Every elementary statement about fields which is true in all but finitely many finite fields is true in $K_{0}$.

(11.1c) $K_{0}$ is an infinite model of the theory of finite fields.

Fried and Völklein [FrV] prove that if $K_{0}$ is a PAC field of characteristic 0 and $G$ is a finite group, then $K=K_{0}(t)$ has a Galois extension $L$ which is regular over $K_{0}$ such that $\mathcal{G}(L / K) \cong G$. The same result without any restriction on the characteristic follows from a theorem of Harbater [Har] (see, [Ja2, Thm 2.6]). However, in none of these results there is a bound on the cardinality of $\operatorname{Ram}(L / K)$ in terms of $G$. The following result suggests such a bound in the case where $K_{0}$ is pseudo finite and $G$ is an l-group such that $l \nmid \operatorname{char}\left(K_{0}\right)$ and $\zeta_{l} \notin K_{0}$.

THEOREM 11.1. Let $K_{0}$ be a pseudo finite field and let $G$ be a group of order $l^{n}$ with a prime $l$. Suppose that $l \neq \operatorname{char}\left(K_{0}\right)$ and $\zeta_{l} \notin K_{0}$. Let $d_{n}=\left[K_{0}\left(\zeta_{l^{n}}\right): K_{0}\right]$. Then there exist absolutely irreducible polynomials $f$, $g \in K_{0}[T, X]$ which are monic and Galois in $X$ such that

(a) $f(T, X)=X^{l^{n}}+a_{1}(T) X^{l^{n}-1}+\cdots+a_{l^{n}}(T)$, with $0<\operatorname{deg}\left(a_{1}(T)\right) \leq$ $\frac{1}{2} n l^{2 n}$, and $\operatorname{deg}\left(a_{i}(T)\right) \leq \operatorname{deg}\left(a_{1}(T)\right), i=1, \ldots, l^{n}$

(b) $f(t, X)$ and $g(t, X)$ have the same splitting field $L$ over $K=K_{0}(t)$;

(c) $L$ is a regular extension of $K_{0}$ and $\mathcal{G}(L / K) \cong G$;

(d) $g \in \mathcal{F}_{k}(L / K)$ where $k=\operatorname{deg}\left(a_{1}(T)\right.$ ) (Notation 11.3) and $\operatorname{deg}_{T} g \leq$ $\left(\frac{1}{2} n l^{2 n}+l^{n}\right)^{2 l^{n}}$

(e) $g_{L}<\frac{1}{2} n l^{2 n}$

(f) $(t)_{\infty}$ totally decomposes in $L$;

(g) $|\operatorname{Ram}(L / K)|=n$ and $\operatorname{deg}(\mathfrak{p})=\left[K_{0}\left(\zeta_{l^{n}}\right): K_{0}\right]$ for each $\mathfrak{p} \in \operatorname{Ram}(L / K)$;

(h) Let $R=K_{0}[t]$ and let $S$ be the integral closure of $R$ in $L$. Then $\operatorname{Disc}(S / R)=\operatorname{gcd}(\operatorname{Disc}(f(t, X)), \operatorname{Disc}(g(t, X)))$. In particular $\operatorname{Ram}(L / K)$ consists of the primes $\neq(t)_{\infty}$ of $L / K_{0}$ that divides both $\operatorname{Disc}(f(t, X))$ and $\operatorname{Disc}(g(t, X))$.

Proof. Let $d_{n}$ be a divisor of $(l-1) l^{n-1}$ and let $2 \leq k \leq \frac{1}{2} n l^{2 n}$. Denote the conjunction of the following elementary statements on $K_{0}$ by $\theta\left(d_{n}, k\right)$ (see [FrJ, proof of Lemma 10.8] for the absolute irreduciblity and [FrJ, Prop. 18.2] for the statement about the Galois group): 
(11.2a) $l \neq \operatorname{char}\left(K_{0}\right), \zeta_{l} \notin K_{0}$, and $d_{n}=\left[K_{0}\left(\zeta_{l^{n}}\right): K_{0}\right]$.

(11.2b) There exist absolutely irreducible polynomials $f, g \in K_{0}[T, X]$ which are monic and Galois in $X$ such that $f(T, X)=X^{l^{n}}+a_{1}(T) X^{l^{n}-1}+$ $\cdots+a_{l^{n}}(T)$ with $\operatorname{deg}\left(a_{i}(T)\right) \leq \operatorname{deg}\left(a_{1}(T)\right)=k, i=1, \ldots, l^{n}$, $f(t, X)$ and $g(t, X)$ have the same splitting field $L$ over $K=K_{0}(t)$ with $\mathcal{G}(L / K) \cong G, g \in \mathcal{F}_{k}(L / K)$, and $d(t)=\operatorname{gcd}(\operatorname{Disc}(f(t, X))$, $\operatorname{Disc}(g(t, X)))$ divides $\operatorname{Disc}(h(t, X))$ for each $h \in \mathcal{F}_{k}(L / K)$, and $d(t)$ has exactly $n$ distinct irreducible divisors, each of them of degree $d_{n}$.

Let $\theta$ be the disjunction of all the above $\theta\left(d_{n}, k\right)$ 's. By Theorem 10.5, for all but finitely many prime powers $q$ the statement $\theta$, with $K_{0}$ replaced by $\mathbb{F}_{q}$, is true in $\mathbb{F}_{q}$. Hence, by $(11.1 \mathrm{~b}), \theta$ is true in $K_{0}$. So, there exist $d_{n}$ and $k$ such that $\theta\left(d_{n}, k\right)$ is true in $K_{0}$. In particular (a), (b), (c), and (d) are true. By Lemma 9.2 (b), $(t)_{\infty}$ totally decomposes in $L$. Hence, by Lemma (10.4a), and with the notation of $(11.2 \mathrm{~b}), \operatorname{Disc}(S / R)=d(t) R$. In particular the primes in $\operatorname{Ram}(L / K)$ correspond to the irreducible divisors of $d(t)$. Hence $(\mathrm{g})$ is true. Finally, by Lemma $8.3,2 g_{L}-2 \leq[L: K](-2+$ $\operatorname{deg}(\operatorname{Ram}(L / K))=l^{n}\left(-2+n d_{n}\right) \leq n l^{2 n}$. So, (e) is also true.

The absolute Galois group $G(F)$ of a field $F$ admits a unique normalized Haar measure. In the following Corollary we use the expression "almost all" with respect to this measure. For each $\sigma \in G(F)$ we denote the fixed field of the unique extension of $\sigma$ to $\tilde{F}$ by $\tilde{F}(\sigma)$.

Corollary 11.2. Let $F$ be a countable Hilbertian field. Then for almost all $\sigma \in G(F)$, the field $K_{0}=\tilde{F}(\sigma)$ satisfies the conclusion of Theorem 11.1.

Proof. By [FrJ, Thm. 18.14], $\tilde{F}(\sigma)$ is a pseudo finite field for almost all $\sigma \in G(F)$. Now apply Theorem 11.1.

\section{$\S 12 . \mathbb{Z}_{l}$-extensions}

We have proved that if $K_{0}$ is a finite field or if $K_{0}=\tilde{F}(\sigma)$ where $F$ is a global field, $\sigma \in G(F)$ is taken at random, and $\zeta_{l} \notin K_{0}$, then for each $l$-group $G$ there exists a Galois extension $L$ of $K=K_{0}(t)$ which is regular over $K_{0}$ such that $\mathcal{G}(L / K) \cong G$. We then say that $G$ is regular over $K_{0}$. 
If $K$ is a function field of one variable over a finite field $K_{0}$ with $l \nmid$ $\operatorname{char}\left(K_{0}\right)$, then it has no Galois extension $L$ which is regular over $K_{0}$ such that $\mathcal{G}(L / K) \cong \mathbb{Z}_{l}$ [GeJ, Thm. 1.1]. In particular, $\mathbb{Z}_{l}$ is not regular over $K_{0}$. Since $K_{0}$ has a unique extension with Galois group $\mathbb{Z}_{l}, K$ has a unique $\mathbb{Z}_{l}$-extension.

Iwasaswa proves [Iwa, Thm. 2] that a number field $K$ has at most $n=[K: \mathbb{Q}]$ linearly independent $\mathbb{Z}_{l}$ extensions. In particular, $\mathbb{Z}_{l}^{n+1}$ is not realizable over $K$. For a finitely generated field $K$ of positive characteristic [GeJ, Thm. 1.1] says that $K$ has exactly one $\mathbb{Z}_{l}$ extension. Thus the realization results of finite $l$-groups do not generalize to pro- $l$ groups.

The goal of this section is to prove an analog of these results for almost all field $\tilde{F}(\sigma)$, where $F$ is a global field.

LEMMA 12.1. Let $K$ be a function field of one variable over a field $K_{0}$ with $\operatorname{char}\left(K_{0}\right) \neq l$. Let $L_{1} / K$ be a cyclic extension of degree $l$ such that $L_{1}$ is regular over $K_{0}$. Suppose that $L_{1}$ is contained in a cyclic extension $L_{n}$ of $K$ of degree $l^{n}$. Suppose that $\mathfrak{p}$ is a prime of $K / K_{0}$ which ramifies in $L_{1}$. Then $\mathfrak{p}$ totally ramifies in $L_{n}$ and its residue field contains $K_{0}\left(\zeta_{l^{n}}\right)$. In particular $\left|\operatorname{Ram}\left(L_{1} \tilde{K}_{0} / K \tilde{K}_{0}\right)\right| \geq\left[K_{0}\left(\zeta_{l^{n}}\right): K_{0}\right]$.

Proof. Denote $L_{n}$ by $L$. The inertia group $I_{\mathfrak{p}}\left(L_{1} / K\right)$ of $\mathfrak{p}$ coincides with $\mathcal{G}\left(L_{1} / K\right)$. Since $\operatorname{res}_{L_{1}}: \mathcal{G}(L / K) \rightarrow \mathcal{G}\left(L_{1} / K\right)$ maps $I_{\mathfrak{p}}(L / K)$ onto $I_{\mathfrak{p}}\left(L_{1} / K\right)$, we have $I_{\mathfrak{p}}(L / K)=\mathcal{G}(L / K)$. In other words, $\mathfrak{p}$ totally ramifies in $L$.

Let $\hat{K}_{\mathfrak{p}}$ be the completion of $K$ at $\mathfrak{p}$ and let $\hat{L}_{\mathfrak{p}}=L \hat{K}_{\mathfrak{p}}$. Then $\hat{L}_{\mathfrak{p}} / \hat{K}_{\mathfrak{p}}$ is a cyclic totally and tamely ramified extension of complete discrete valuation fields. Hence $\zeta_{l^{n}} \in \hat{K}_{\mathfrak{p}}\left[\mathrm{CaF}\right.$, p. 32] and therefore $\zeta_{l^{n}} \in \bar{K}_{\mathfrak{p}}$. It follows that there are at least $\left[K_{0}\left(\zeta_{l^{n}}\right): K_{0}\right]$ distinct primes $\tilde{\mathfrak{p}}$ of $K \tilde{K}_{0}$ which lie over $\mathfrak{p}$. Each of them totally ramifies in $L \tilde{K}_{0}$ and therefore also in $L_{1} \tilde{K}_{0}$. Thus $\left|\operatorname{Ram}\left(L_{1} \tilde{K}_{0} / K \tilde{K}_{0}\right)\right| \geq\left[K_{0}\left(\zeta_{l^{n}}\right): K_{0}\right]$.

Proposition 12.2. Let $K$ be a function field of one variable over $a$ field $K_{0}$ of characteristic $\neq l$ such that $\left[K_{0}\left(\zeta_{l^{n}}\right): K_{0}\right]$ is unbounded. Then $K$ has no ramified Galois extension $L$ which is regular over $K_{0}$ such that $\mathcal{G}(L / K) \cong \mathbb{Z}_{l}$. In particular, $\mathbb{Z}_{l}$ is not regular over $K_{0}$.

Proof. Assume that there exists $L$ as above. Then $L$ is the ascending union of Galois extensions $L_{n}$ of $K$ with $\mathcal{G}\left(L_{n} / K\right) \cong \mathbb{Z} / l^{n} \mathbb{Z}$. Each $L_{n}$ is a regular extension of $K$ and for large $m, L_{m+1} / L_{m}$ is ramified. Replace $K$ 
by such $L_{m}$, if necessary to assume that $L_{1} / K$ is ramified. By Lemma 12.1, $\left[K_{0}\left(\zeta_{l^{n}}\right): K_{0}\right] \leq\left|\operatorname{Ram}\left(L_{1} \tilde{K}_{0} / K \tilde{K}_{0}\right)\right|$, a contradiction.

Finally recall that if $K=K_{0}(t)$, then $L_{1} / K$ is ramified [FrJ, Prop. 2.15]. Hence, $K$ admits no $\mathbb{Z}_{l}$-extension $L$ which is regular over $K_{0}$.

Proposition 12.3. Let $F$ be a global field of characteristic $\neq l$. Then for almost all $\sigma \in G(F)$, the group $\mathbb{Z}_{l}$ is not regular over $\tilde{F}(\sigma)$.

Proof. Let $N=F\left(\zeta_{l}, \zeta_{l^{2}}, \zeta_{l^{3}}, \ldots\right)$. Then $N=K L$ with $K \cap L=$ $F, \mathcal{G}(K / F) \cong \mathbb{Z}_{l}$, and $\mathcal{G}(L / F)=A$ is a finite group. Thus $\mathcal{G}(N / F)=$ $\mathcal{G}(N / K) \times \mathcal{G}(N / L)$. If $H$ is a finite subgroup of $\mathcal{G}(N / F)$, then its projection on $\mathcal{G}(N / L)$ is also finite and therefore trivial. Thus $H \leq \mathcal{G}(N / K)$. It follows that if for some $\sigma \in G(F)$ the degree $\left[\tilde{F}(\sigma)\left(\zeta_{l^{n}}\right): \tilde{F}(\sigma)\right]$ is bounded, then $\mathcal{G}(N / N \cap \tilde{F}(\sigma))$ is finite and therefore $K \subseteq \tilde{F}(\sigma)$. Since $K / F$ is infinite, almost no $\sigma \in G(F)$ satisfies the latter condition. Hence, for almost all $\sigma \in G(F),\left[\tilde{F}(\sigma)\left(\zeta_{l^{n}}\right): \tilde{F}(\sigma)\right]$ is unbounded. For each of these $\sigma$, Proposition 12.2 , asserts that $\mathbb{Z}_{l}$ is not regular over $K_{0}$.

\section{Appendix. Effective form of the Chebotarev density theorem}

Lemma 5.2 uses an effective form of the Chebotarev density theorem for function fields. One may find such a form in [FrJ, §5.4] and in [HKo]. Unfortunately, the proof of [FrJ, Prop 5.16] applies [FrJ, Lemma 5.14] in a faulty way. Indeed, on [FrJ, page 63 , line -3$] d$ should be replaced by $m d$. The same mistake occurs in [HKo]. We therefore take this opportunity to correct the mistake and at the same time to improve the estimate of [FrJ, Prop 5.16].

Data 13.1. We fix the following notation for the whole section.

$$
\begin{aligned}
& q=\text { a power of a prime number } \\
& t=\text { a transcendental element over } \mathbb{F}_{q} \\
& K=\text { a finite separable extension over } \mathbb{F}_{q}(t) \\
& \text { which is regular over } \mathbb{F}_{q} \\
& d=\left[K: \mathbb{F}_{q}(t)\right] \quad \\
& L=\text { a finite Galois extension of } K \\
& \mathbb{F}_{q^{n}} \quad \text { the algebraic closure of } \mathbb{F}_{q} \text { in } L \\
& \mathbb{P}(K)=\text { the set of all prime divisors of } K / \mathbb{F}_{q}
\end{aligned}
$$




$$
\begin{aligned}
\mathbb{P}^{\prime}(K) & =\left\{\mathfrak{p} \in \mathbb{P}(K) \mid \mathfrak{p} \text { is unramified over } \mathbb{F}_{q}(t) \text { or in } L\right\} \\
\mathbb{P}_{k}(K) & =\{\mathfrak{p} \in \mathbb{P}(K) \mid \operatorname{deg}(\mathfrak{p})=k\} \\
\mathbb{P}_{k}^{\prime}(K) & =\left\{\mathfrak{p} \in \mathbb{P}^{\prime}(K) \mid \operatorname{deg}(\mathfrak{p})=k\right\} \\
\mathcal{C} & =\text { a conjugacy class in } \mathcal{G}(L / K) ; c=|\mathcal{C}| \\
C_{k}(L / K, \mathcal{C}) & =\left\{\mathfrak{p} \in \mathbb{P}_{k}^{\prime}(K) \mid\left(\frac{L / K}{\mathfrak{p}}\right)=\mathcal{C}\right\}
\end{aligned}
$$

Our first result improves [FrJ, Lemma 5.14].

LEMMA 13.2. Suppose that $L=K \mathbb{F}_{q^{n}}, \mathcal{C}=\{\tau\}$, and $\left.\tau\right|_{\mathbb{F}_{q^{n}}}=\varphi$. Then

$$
\left|\# C_{1}(L / K, \mathcal{C})-q\right|<2\left(g_{L} \sqrt{q}+g_{L}+d\right) .
$$

Proof. Note first that $C_{1}(L / K, \mathcal{C})=\mathbb{P}_{1}^{\prime}(K)$ and that each $\mathfrak{p} \in \mathbb{P}(K)$ is unramified in $L$. Thus, $\mathbb{P}_{1}(K) \backslash C_{1}(L / K, \mathcal{C})$ consists exactly of all prime divisors of Different $\left(K / \mathbb{F}_{q}(t)\right)$. By the Riemann-Hurwitz genus formula, $\operatorname{deg}\left(\operatorname{Different}\left(K / \mathbb{F}_{q}(t)\right)=2\left(g_{K}+d-1\right)[\mathrm{FrJ}\right.$, P. 24]. By Weil's theorem $\left|\# \mathbb{P}_{1}(K)-(q+1)\right| \leq 2 g_{K} \sqrt{q}\left[\right.$ FrJ, Thm. 3.14]. Hence, $\left|\# C_{1}(L / K, \mathcal{C})-q\right| \leq$ $2 g_{K} \sqrt{q}+1+2\left(g_{K}+d-1\right)$. Since $g_{K}=g_{L}$, this proves $(13.1)$.

Next we improve [FrJ, Lemma 5.15]. Here we use the notation ' $a$ pd $b$ ' to mean ' $a$ properly divides $b$ '.

LEMMA 13.3. Let $K^{\prime}$ be an extension of $K$ of degree $k m$ which contains $\mathbb{F}_{q^{k}}$. For each $\mathfrak{q} \in \mathbb{P}\left(K^{\prime}\right)$ we denote the prime of $K$ which lies under $\mathfrak{q}$ by $\mathfrak{q}_{K}$. Then

$$
\#\left\{\mathfrak{q} \in \mathbb{P}\left(K^{\prime}\right) \mid \operatorname{deg}\left(\mathfrak{q}_{K}\right) \operatorname{pd} k\right\} \leq m\left(q^{k / 2}+\left(3 g_{K}+1\right) q^{k / 4}\right) .
$$

Proof. If $j \mid k$ and $\mathfrak{p} \in \mathbb{P}(K)$, then $\mathbb{F}_{q^{j}} \subseteq \mathbb{F}_{q^{k}}$ and therefore $\mathfrak{p}$ decomposes in $K \mathbb{F}_{q^{j}}$ into $j$ prime divisors of degree 1 . Each of them has exactly one extension to $K \mathbb{F}_{q^{k}}$ and the latter decomposes in $K^{\prime}$ into at most $m$ prime divisors. Hence, by Weil's theorem

$$
\begin{aligned}
\#\left\{\mathfrak{q} \in \mathbb{P}\left(K^{\prime}\right) \mid \operatorname{deg}\left(\mathfrak{q}_{K}\right) \operatorname{pd} k\right\} & \leq m \sum_{j \leq k / 2} \frac{1}{j}\left|\mathbb{P}_{1}\left(K \mathbb{F}_{q^{j}}\right)\right| \\
& \leq m \sum_{j \leq k / 2} \frac{1}{j}\left(q^{j}+2 g_{K} q^{j / 2}+1\right) .
\end{aligned}
$$


Induction on $k$ shows that for $q \geq 2$

$$
\sum_{j \leq k / 2} \frac{q^{j}}{j} \leq q^{k / 2}
$$

A direct check for $k \leq 5$ and an induction for $k \geq 6$ shows that for $q \geq \frac{9}{7}$

$$
\sum_{j \leq k / 2} \frac{q^{j}}{j} \leq \frac{3}{2} q^{k / 2}
$$

If $q$ is a power of a prime, then $q^{1 / 2} \geq \sqrt{2} \geq 9 / 7$. Hence, (13.2) is a consequence of (13.3), (13.4), and (13.5).

Finally we improve [FrJ, Prop. 5.16].

Proposition 13.4. In the notation of Data 13.1 let a be a positive integer and let $\tau$ be an element of $\mathcal{C}$ such that $\left.\tau\right|_{\mathbb{F}_{q^{n}}}=\varphi^{a}$. Let $k$ be a positive integer such that $k \equiv a \bmod n$. Then

$$
\begin{aligned}
& \left|\# C_{k}(L / K, \mathcal{C})-\frac{c}{k m} q^{k}\right| \\
& \quad<\frac{c}{k m}\left[\left(m+2 g_{L}\right) q^{k / 2}+m\left(3 g_{K}+1\right) q^{k / 4}+2\left(g_{L}+d m\right)\right] .
\end{aligned}
$$

Proof. Let $n^{\prime}=n k \cdot \operatorname{ord}(\tau)$ and extend $L$ to $L^{\prime}=L \mathbb{F}_{q^{n^{\prime}}}$. Then $\left[L^{\prime}\right.$ : $\left.K \mathbb{F}_{q^{n^{\prime}}}\right]=\left[L: K \mathbb{F}_{q^{n}}\right]=m$. Since $k \equiv a \bmod n$ there .exists $\tau^{\prime} \in \mathcal{G}\left(L^{\prime} / K\right)$ such that $\left.\tau^{\prime}\right|_{L}=\tau$ and $\left.\tau^{\prime}\right|_{\mathbb{F}_{q^{n^{\prime}}}}=\varphi^{k}$. Then ord $\left(\tau^{\prime}\right)=\operatorname{lcm}\left(\operatorname{ord}(\tau), \operatorname{ord}\left(\varphi^{k}\right)\right)=$ $\operatorname{lcm}\left(\operatorname{ord}(\tau),\left[\mathbb{F}_{q^{n^{\prime}}}: \mathbb{F}_{q^{k}}\right]\right)=\operatorname{lcm}(\operatorname{ord}(\tau), n \cdot \operatorname{ord}(\tau))=n \cdot \operatorname{ord}(\tau)$. Denote the conjugacy class of $\tau^{\prime}$ in $\mathcal{G}\left(L^{\prime} / K\right)$ by $\mathcal{C}^{\prime}$. By [FrJ, Lemma 5.12(c)], $C_{k}\left(L^{\prime} / K, \mathcal{C}^{\prime}\right)=C_{k}(L / K, \mathcal{C})$.

Denote the fixed field of $\tau^{\prime}$ in $L^{\prime}$ by $K^{\prime}$. Then $K^{\prime} \cap \mathbb{F}_{q^{n^{\prime}}}=K^{\prime} \cap \tilde{\mathbb{F}}_{q}=\mathbb{F}_{q^{k}}$ 
and $K^{\prime} \mathbb{F}_{q^{n^{\prime}}}=L^{\prime}$

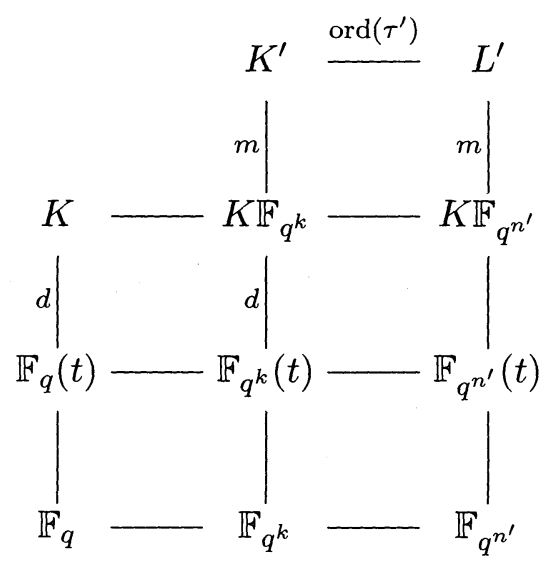

Then $\left[K^{\prime}: K \mathbb{F}_{q^{k}}\right]=\left[L: K \mathbb{F}_{q^{n^{\prime}}}\right]=m$ and therefore $\left[K^{\prime}: \mathbb{F}_{q^{k}}(t)\right]=d m$. By [FrJ, Cor. 5.11] applied to $L^{\prime}, K, \mathcal{C}^{\prime},\left\{\tau^{\prime}\right\}, k$ instead of $F, E, \mathcal{C}, \mathcal{C}^{\prime}, r$,

$$
\left|C_{k}\left(L^{\prime} / K, \mathcal{C}^{\prime}\right)\right|=\frac{c}{\left[K^{\prime}: K\right]}\left|C_{1}\left(L^{\prime} / K^{\prime},\left\{\tau^{\prime}\right\}\right) \backslash\left\{\mathfrak{q} \in \mathbb{P}\left(K^{\prime}\right) \mid \operatorname{deg}\left(\mathfrak{q}_{K}\right) \operatorname{pd} k\right\}\right|
$$

Since $\left[K^{\prime}: K\right]=k m$, we have by Lemma 13.3 ,

$$
\begin{aligned}
\mid \# C_{k}\left(L^{\prime} / K, \mathcal{C}^{\prime}\right)- & \frac{c}{k m} \# C_{1}\left(L^{\prime} / K^{\prime},\left\{\tau^{\prime}\right\}\right) \mid \\
& \leq \frac{c}{k m} \#\left\{\mathfrak{q} \in \mathbb{P}\left(K^{\prime}\right) \mid \operatorname{deg}\left(\mathfrak{q}_{K}\right) \operatorname{pd} k\right\} \\
& \leq \frac{c}{k m} \cdot m\left(q^{k / 2}+\left(3 g_{K}+1\right) q^{k / 4}\right)
\end{aligned}
$$

By Lemma 13.2 applied to $K^{\prime}, L^{\prime}, n^{\prime}, \tau^{\prime}, q^{k}$ instead of to $K, L, n, \tau, q$

$$
\left|\# C_{1}\left(L^{\prime} / K^{\prime},\left\{\tau^{\prime}\right\}\right)-q^{k}\right|<2\left(g_{L^{\prime}} q^{k / 2}+g_{L^{\prime}}+d m\right) .
$$

Multiply $(13.8)$ by $\frac{c}{k m}$ and replace $g_{L^{\prime}}$ by $g_{L}$. Then replace $C_{K}\left(L^{\prime} / K, \mathcal{C}^{\prime}\right)$ in $(13.7)$ by $C_{k}(L / K, \mathcal{C})$. Finally combine the two inequalities obtained in this way to $(13.6)$.

Corollary 13.5. If in the situation of Proposition 13.4

$$
k \log q \geq \max \left\{2 \log \left(g_{L}+d m\right), 4 \log \left(3 g_{K}+1\right)\right\}
$$

then

$$
\left|\# C_{k}(L / K, \mathcal{C})-\frac{c}{k m} q^{k}\right|<\frac{2 c}{k m}\left(m+g_{L}+1\right) q^{k / 2}
$$




$$
\begin{gathered}
\text { Proof. By }(13.9), 3 g_{K}+1 \leq q^{k / 4} \text { and } g_{L}+d m \leq q^{k / 2} . \text { Hence } \\
\left(m+2 g_{L}\right) q^{k / 2}+m\left(3 g_{K}+1\right) q^{k / 4}+2\left(g_{L}+d m\right) \leq\left(m+2 g_{L}+m+2\right) q^{k / 2}
\end{gathered}
$$

Now combine this inequality with (13.6) to get (13.10).

\section{REFERENCES}

[Ax] J. Ax, The elementary theory of finite fields, Annals of Mathematics, 88 (1968), 239-271.

[CaF] J. W. S. Cassels and A. Fröhlich, Algebraic Number Theory, Academic Press, London, 1967.

[FrJ] M. D. Fried and M. Jarden, Field Arithmetic, Ergebnisse der Mathematik (3), 11, Springer, Heidelberg (1986).

[FrV] M. D. Fried and H. Völklein, The inverse Galois problem and rational points on moduli spaces, Mathematische Annalen 290 (1991), 771-800.

[GeJ] W.-D. Geyer and C. U. Jensen, Extension prodiédrales, C. R. Acad. Sci. Paris Sér. I, 319 (1994), 1241-1244.

[HaV] D. Haran and H. Völklein, Galois groups over complete valued fields, Isreal Journal of Mathematics, 93 (1996), 9-27.

[Hoe] Klaus Hoechsmann, Zum Einbettungsproblem, Journal für die reine und ungewandte Mathematik, 229 (1968), 81-106.

[HKo] F. Halter-Koch, Der Čebotarev'sche Dichtigkeitsatz und ein analogon zum Dirichlet'schen Primzahlsatz für algebraische Funktionenkörper, manuscripta mathematica, 72 (1991), 205-211.

[Ja1] M. Jarden, Elementary statements over large algebraic fields, Transactions of AMS, 164 (1972), 67-91.

[Ja2] M. Jarden, The inverse Galois problem over formal power series fields,, Israel Journal of Mathematics, 85 (1994), 263-275.

[Koc] H. Koch, l-Erweiterungen mit vorgegebener Verzweigunsstellen, Journal für die reine und angewandte Mathematik, 219 (1965), 30-61.

[Lan] S. Lang, Algebra, Addison-Wesley, Reading, 1970.

[La2] S. Lang, Algebraic Number Theory, Addison-Wesley, Reading, 1970.

[La3] S. Lang, Introduction to algebraic geometry, Interscience Publishers, New York, 1958.

[La4] S. Lang, Algebra, Second Edition, Addison-Wesley, Menlo Park, 1984.

[Neu] J. Neukirch, Class field theory, Grundlehren der mathematischen Wissenschaften, 280 (1985), Springer, Berlin.

[RCV] Rzedowski-Calderón and Villa-Salvador, Automorphisms of congruence function fields, Pacific Journal of Mathematics, 150 (1991), 167-178.

[Rei] H. Reichardt, Konstruktion von Zahlkörpern mit gegebener Galoisgruppe von Primzahlpotenzordnung, Journal für die reine und angewandte Mathematik, $17 \mathbf{7}$ (1937), 1-5. 
[Sch] Arnold Scholz, Konstruktion algebraischer Zahlkörper mit beliebiger Gruppe von Primzahlpotenzordnung I, Mathematische Zeitschrift, 42 (1936), 161-188.

[Se1] J.-P. Serre,, Topics in Galois Theory, Jones and Barlett, Boston, 1992.

[Se2] J.-P. Serre, Corps Locaux, Hermann, Paris, 1962.

[Sh1] I. R. Shafarevich, On the construction of fields with a given Galois group of order $l^{a}$, Izv. Akad. Nauk, 18 (1954), 261-296, = Collected Mathematical Papers 69-97, Springer, Berlin, 1989.

[Sh2] I. R. Shafarevich, Factors of descending central series, Mathematical Notes, 45 (1989), 262-264.

\section{Wulf-Dieter Geyer}

Mathematisches Institut, Universität Erlangen

Bismarckstraße $1 \frac{1}{2}, 91054$, Germany

geyer@mi.uni-erlangen.de

Moshe Jarden

School of Mathematical Sciences, Tel Aviv University

Ramat Aviv, Tel Aviv 69978, Israel

jarden@math.tau.ac.il 